DOE

3-07-08

THE MIND INSTITUTE - MENTAL ILLNESS AND NEUROSCIENCE DISCOVERY GRANT

Progress Report for the Period 04/01/07 - 03/31/2008

Date Prepared - March 7, 2008

Assembled by Melissa Hilleary

PREPARED FOR THE UNITED STATES DEPARTMENT OF ENERGY / OFFICE OF BIOLOGICAL AND ENVIRONMENTAL RESEARCH PROGRAM

Work Performed Under Grant No. DE-FG02-07ER64415 
Progress Report Overview

National Projects

$1 \quad$ MIND Clinical Imaging Consortium

$2 \quad$ FIRST Program

o University of Minnesota

o Massachuttes General Hospital 10

o University of New Mexico 13

$3 \quad$ MEG Consortium

$4 \quad$ Neurodevelopment Research Program

4.1 Neurodevelopment Research Program: Diagnosis of Epilepsy and Epileptogenesis After Brain Injury in infants

4.2 Neurodevelopment Research Program: Multisensory Integration and Development of Mirror Neurons in Children with Autism: In Search of an Early Biological Marker with MEG

Individual Projects

$5 \quad$ Direct Detection of Neuronal Actity Using MRI

$6 \quad$ Knowledge Environment

7 Application of Diffusion Tensor Imaging to Characterize Subjects with Schizophrenia

$8 \quad$ Auditory Sensory Gating using MEG

9 Investigation of the Neural Mechanisms Underlying Facial Emotion Recognition

10 Effect of Cigarettes on fMRI Studies of Schizophrenia Patients

11 Multimodal Assessment of Lateralized Hippocampal Function in Schizophrenia

12 Structural Mechanisms of Convergent and Divergent Cognitive Processes in Humans 
14 Application of Novel Neuroimaging Techniques to the Study of Dementia

15 A Multimodal Study of Sensory Gating in Schizophrenia

16 Attentional Functioning in Normal and Neuropsychiatric Populations

17 Neurochemistry of Brain Activity in Response to Pain

18 Application of Neuroimaging Techniques to Improve Methods of Matching Children with Dyslexia to Reading Intervention

19 Constraint Therapy For Children With Hemiplegic Cerebral Palsy Cortical Visual Impairment in Children with Cerebral Palsy

$20 \quad$ High Field fMRI

21 Improved Methodology and Hardware for C13 MRS studies

22 Development Of Clinical Analysis Tools For MEG Data Gathered On Patients With Epilepsy

60

$24 \quad$ MIND Mobile Imaging Core
A Publications
B Funded Grant Applications
B1
C Pending Grant Applications
C1
D Equipment Purchases
D1
E Patents and Inventions 


\section{Overview:}

The U.S. Department of Energy (DOE) awarded under Grant No. DE-FG02-07ER64415 funding in the amount of \$7,000,579 to the Mental Illness and Neurodiagnostic Discovery (MIND) Institute. This award was in response to a competitively reviewed proposal submitted by the Institute to continue its pioneering research in technology development for better understanding of brain function and diagnosis of mental illness and neurological disorders. MIND's initial proposal was for a nine-month period, from $01 / 01 / 2007$ to $09 / 30 / 2007$. In the award to MIND, the nine-month amount requested was held static, however the period of performance was expanded to one year, from 04/01/2007 to 03/31/2008. This required some adjustments in the proposed research plan. Selected projects were reduced in scope and others withdrawn in order to work within the funding allotment. The projects that went forward are presented below.

As in past years, the scientific and technological programs of the MIND reflect DOE missions in basic science and associated instrumentation, computational modeling, and experimental techniques. The technical goals to develop and apply integrated, multi-modality functional imaging techniques derive from a decade of DOE-supported research and technology development.

In the past year, MIND pursued three National Programs. The FIRST (Functional Imaging for Research and Schizophrenia Treatment) Program, led by Dr. Charles Schulz, M.D., University of Minnesota, links multi-modal brain imaging, genetics and pattern recognition to develop a definitive diagnostic tool for mental illness. The Neurodevelopmental Program, led by Dr. Yoshio Okada, University of New Mexico, pursued development of novel diagnostic techniques based on MRI, NIRS, MEG, and EEG for studying brain development and application of these techniques to understanding the consequence of brain injury in babies and children with autism. The MEG Tech Program led by Dr. Michael Weisend, The MIND Institute, is focused on developing cross-platform (CTF, 4-D, Neuromag) openly distributed software that is fully characterized for sensitivity, accuracy and reliability.

MIND also requested support to match young investigators with senior scientists in the larger MIND Institute network. These collaborative efforts included improved diagnostic imaging tools for traumatic brain injury, integration of genetic, morphometric and behavioral data, and synthesis of spectroscopic and diffusion tensor imaging. And finally, MIND continued to build on critical developments in shared infrastructure, including construction of the most comprehensive neuroinformatics capability in the country to facilitate data mining and data sharing.

This document has been prepared to respond to all required reporting criteria. Given that this report encompasses multiple projects, selected information has been consolidated in appendices at the end of the document, including all publications, grants awarded, pending proposal, significant equipment purchases and invention/patent applications.

As a closing note, the MIND Institute changed its name to the Mind Research Network effective $02 / 11 / 2008$. This report refers to the organization under the old former name, in consideration of the fact it was operating under that designation during most of the reporting time frame covered (04/01/200703/31/2008). 
National Programs 


\section{Project 1: MIND Clinical Imaging Consortium \\ Investigator: S. Charles Schulz \\ Period of Report: 4/1/07-3/31/08}

\section{Abstract:}

Through past DOE funding, the MIND Research network has funded a national consortium effort that used multi-modal neuroimaging, genetics, and clinical assessment of subjects to study schizophrenia in both first episode and persistently ill patients. Although active recruitment of research participants is complete, this consortium remains active and productive in terms of analysis of this unique multi-modal data collected on over 320 subjects.

\section{Objectives and Findings:}

We have made significant progress the past 12 months and have seen scientific productivity increase dramatically.

For analysis of functional imaging (fmr) we have completely analyzed the Sternberg and Oddball tasks, as well as the sensory motor and behavior tasks. Significant quality assurance and curation has been performed on the fmr tasks. Several manuscripts that summarize the first wave of results have been submitted or are in final stages of preparation. Several additional manuscripts are planned over the coming months.

For analysis of anatomical imaging we have completed the processing of both freesurfer and BRAINS pipelines on the structural imaging (sMR). A manuscript summarizing the overall group effects using freesurfer is in preparation. We have also completed processing white matter region scalars on the diffusion-weighted imaging data (DTI). A manuscript summarizing the overall group effects found between patients and controls for white matter scalars is in final stages of preparation. Going forward, we plan to look at tensor based statistics and tractography through collaboration with the NA-MIC group.

For genetic analysis we have completed the analysis of 100 SNPs. These results are available to our investigators; moreover, since cell lines were collected, we have the capability of running significantly more in future work. An R01 is under review that would fund expanded genetic analyses.

A manuscript that contains an overview of the consortium, summarizes all of the tasks that were used, strategies for analyses, strategies for recruiting, pitfalls to avoid, and lessons learned is in preparation.

Although we plan to continue active mining of this data, we have submitted an R01 grant on which through collaboration with the BIRN community, we will share the MIND Clinical Imaging Consortium data sources with the scientific community.

\section{Manuscripts in Progress:}

Examination of smoking status as a possible confound in the BOLD response in patients with schizophrenia; Leonard Leyba, Andrew R. Mayer, Randy L. Gollub, Nancy C. Andreasen, Vincent P. Clark (Submitted to Schizophrenia Research 2/08)

MTHFR 677CàT genotype disrupts prefrontal and dopaminergic function in schizophrenia; Joshua L. Roffman, Randy L. Gollub, Vince D. Calhoun, Thomas H. Wassink, Anthony P. Weiss, Beng C. Ho, 
Tonya White, Jill Fries, Nancy C. Andreasen, Donald C. Goff, Dara S. Manoach (Submitted Nature Neuroscience 3/08)

An analysis of the fMRI BIRN and MIND studies shows a dysmodulation of functional networks in schizophrenia during working memory; D. Kim, D. S. Manoach, J. Turner, G. Brown, A. Belger, H. J. Bockholt, V. P. Clark, J. M. Ford, R. Gollub, J Lauriello, D. Mathalon, T. White, C. Wible, D. Leary, B. Mueller, K. Lim, N. Andreasen, S. Potkin, V. D. Calhoun (Submitted to Archives of General Psychiatry 2/08)

Real-time Web-scale Image Annotation for Semantic-based Retrieval of Neuropsychiatric Research Images H. Jeremy Bockholt, Josef Ling, Mark Scully, Vincent A. Magnotta, Tonya White, Kelvin O. Lim, Randy Gollub, and Vince D. Calhoun (submission to Neuroinformatics 3/08).

Global White matter abnormalities in schizophrenia: A multicenter diffusion tensor imaging study T White, V Magnotta, J Bockholt, S Williams, B Mueller, BC Ho, RE Jung, V Clark, SC Schulz, J Lauriello, NC Andreasen, R L Gollub, V Calhoun, K O Lim (Planned submission to the Archives of General Psychiatry, 3/08).

\section{Presentations and Posters:}

Wallace S, Gollub RL, Norman K, Vangel G, Manoach V, Lim KO, Lauriello J, Andreasen N, Kunkel L, Diagnostic utility of neurological exam abnormalities in schizophrenia, Society for Neuroscience, 2007.

Machado GR, Clark VP, Gollub RL, Lauriello J, Magnotta V, White T, Calhoun VD, Probing schizophrenia with a sensorimotor task: large-scale $(n=273)$ independent component analysis of first episode and chronic schizophrenia patients, Society for Neuroscience, 2007.

Gollub RL, Manoach DS, Fries J, Vangel MG, White T, Andreasen N, Calhoun VD, Multi-site fMRI study of load dependent DLPFC activation in schizophrenia, Society for Neuroscience, 2007.

Lim KO, Bockholt J, Magnotta V, Gollub RL, Andreasen N, White T, Schultz C, White matter abnormalities in first episode and chronic schizophrenia using diffusion tensor imaging in the MIND Clinical Imaging Consortium Study, International Congress on Schizophrenia Research, 2007.

Clark V, Manoach D, Gollub RL, Ho BC, Lim KO, Burge J, Lane T, Lesnik P, Calhoun VD, Andreasen NC, A multi-site fMRI study of schizophrenia: effects of illness type and duration on brain function and connectivity, International Congress on Schizophrenia Research, 2007.

Bockholt HJ, Ling J, Scully M, Magnotta VA, Gollub RL, White T, Schulz SC, Lauriello J, Andreasen NC, MIND Clinical Imaging Consortium as a case study of novel multi-center neuroinformatics software, International Congress on Schizophrenia Research, 2007

Morrow EM, Wallace S, Pacheco J, Bockholt J, White T, Ho B, Kuperberg G, Magnotta V, Lim KO, Seidman L, Goff D, Andreasen N, Schulz C, Fischl B, Gollub RL, Structural brain study across lifetime of schizophrenia using computer automated quantification of subcortical volumes in the MIND multisite study, International Congress on Schizophrenia Research, 2007.

J. Lauriello, J. Bustillo1, S. C. Schulz, N. Andreasen, R. Gollub, B. C. Ho, V. Clark, J. Bockholt, K. O. Lim. Overview of the MIND Imaging Consortium, International Congress on Schizophrenia Research, 2007. 
M. Benavidez, V. P. Clark, G. Kuperberg, K. Lim, and V. D. Calhoun, "Functional Networks Identified in an Auditory Oddball Task of Chronic and First Episode Schizophrenia Patients ( $N=261)$ Collected from the MIND Clinical Imaging Consortium," in Proc. Society for Neuroscience San Diego, CA, 2007.

H. J. Bockholt, J. Turner, D. L. Johnson, V. D. Calhoun, D. N. Greve, A. W. Toga, C. G. Wible, K. Lim, B. Mueller, J. Lauriello, D. S. O'Leary, G. H. Glover, J. T. Voyvodic, G. McCarthy, J. M. Ford, S. Potkin, and FBIRN, "Morphometric analysis of a multi-site study of schizophrenia using freesurfer," in Proc. Society for Neuroscience San Diego, CA, 2007.

V. P. Clark, D. S. Manoach, R. L. Gollub, B. C. Ho, K. Lim, J. Burge, T. Lane, P. Lesnik, V. D. Calhoun, and N. C. Andreasen, "A Multi-site fMRI Study of Schizophrenia: Effects of Illness type and Duration on Brain Function and Connectivity," in Proc. ICOSR, 2007.

O. Demirci and V. D. Calhoun, "Detection of Schizophrenia using fMRI Data via Projection Pursuit," in Proc.MLSP, 2007.

R. L. Gollub, D. S. Manoach, J. Fries, M. Vangel, T. White, N. C. Andreasen, and V. D. Calhoun, "Multisite fMRI study of DLPFC activation differences between subjects with schizophrenia and controls," in Proc. Society for Neuroscience San Diego, CA, 2007.

G. Machado, M. Juarez, V. P. Clark, R. L. Gollub, V. Magnotta, T. White, and V. D. Calhoun, "Probing Schizophrenia With A Sensorimotor Task: Large-Scale (N=273) Independent Component Analysis Of First Episode And Chronic Schizophrenia Patients," in Proc. Society for Neuroscience San Diego, CA, 2007.

J. Roffmann, R. L. Gollub, D. S. Manoach, and V. Calhoun, "Interactive effects of MTHFS C677T and COMT Val158Met on executive function and prefrontal activation in schizophrenia," in Proc.SIRS, 2007.

T. Van Erp, J. B. Segall, J. Turner, D. N. Greve, A. W. Toga, C. G. Wible, K. Lim, B. Mueller, J. Lauriello, D. S. O'Leary, G. H. Glover, G. Brown, J. T. Voyvodic, G. McCarthy, S. Potkin, and V. D. Calhoun, "Voxel-Based Morphometric analysis of a multi-site study on schizophrenia," in Proc. Society for Neuroscience San Diego, CA, 2007.

J. Liu, L. Xu, A. Caprihan, and V. Calhoun, "Extracting Principle Components For Discriminant Analysis Of Fmri Images," in Proc. ICASSP, 2008.

A. Michael, J. Fries, S. Baum, B. C. Ho, N. C. Andreasen, and V. D. Calhoun, "A Method to Analyze Correlations between Multiple Brain Imaging Tasks to Characterize Schizophrenia," in Proc. IEEE SSIAI, Santa Fe, NM, 2008.

R. Silva and V. D. Calhoun, "Identification of Brain Image Biomarkers by Optimized Selection of Multimodal Datasets," in Proc.ISMRM, 2008.

R. F. Silva and V. D. Calhoun, "Identification of Brain Imaging Biomarkers by Optimized Selection of Multimodal Independent Components," in Proc. IEEE SSIAI, Santa Fe, NM, 2008.

J. Sui and V. D. Calhoun, "A Method for Group Difference Enhancement by Constraining Mixing Coefficients of ICA Framework," in Proc.ISMRM, 2008.

J. Sui, J. Liu, L. Wu, A. Michael, L. Xu, T. Adali, and V. D. Calhoun, "A Constrained Coefficient ICA Algorithm For Group Difference Enhancement," in Proc. ICASSP, 2008. 
O. Demirci, V. P. Clark, and V. D. Calhoun, "A Projection Pursuit Algorithm to Classify Individuals Using fMRI Data: Application to Schizophrenia," NeuroImage, In Press.

H. J. Bockholt, J. Ling, M. Scully, A. Scott, S. Lane, V. Magnotta, T. White, K. Lim, R. L. Gollub, and V. D. Calhoun, "Real-time Web-scale Image Annotation for Semantic-based Retrieval of Neuropsychiatric Research Images," in Proc.HBM, Submitted.

H. J. Bockholt, S. Williams, M. Scully, V. Magnotta, R. L. Gollub, J. Lauriello, K. Lim, T. White, R. Jung, S. C. Schulz, N. C. Andreasen, and V. D. Calhoun, "The MIND Clinical Imaging Consortium as an application for novel comprehensive quality assurance procedures in a multi-site heterogeneous clinical research study," in Proc.HBM, Submitted.

V. Potluru, S. M. Plis, and V. D. Calhoun, "Multiplicative updates for non-negative kernel SVM," in Proc. AAAI, Chicago, IL, Submitted.

S. C. Schultz, A. Georgopoulos, R. L. Gollub, N. C. Andreasen, B. C. Ho, J. Lauriello, and V. D. Calhoun, "Linear Discriminate Analysis Applied to a Multicenter First Episode Schizophrenia Sample," in Winter Workshop on Schizophrenia, Submitted.

J. Sui, T. Adali, V. P. Clark, G. Pearlson, and V. D. Calhoun, "A Method for Group Difference Enhancement by Constraining the Mixing Coefficients in an ICA Framework," NeuroImage, Submitted.

T. van Erp, M.-C. Chiang, D. Sun, M.-C. E. Hardt, J. H. Bockholt, J. A. Turner, V. D. Calhoun, H. J. Johnson, D. N. Greve, S. Williams, D. O'Leary, J. Lauriello, C. G. Wible, K. O. Lim, B. A. Mueller, G. G. Brown, J. Voyvodic, G. McCarthy, D. Mathalon, J. M. Ford, S. G. Potkin, T. D.

Cannon, P. M. Thompson, A. W. Toga, and F. ; "3D Pattern of Brain Abnormalities in Chronic Schizophrenia Visualized Using Tensor-Based Morphometry: a Multi-Site Structural Imaging Study," in Proc.HBM, Submitted.

Tonya White, Vince Magnotta, Jeremy Bockholt, Sumner Williams, Ron Pierson, Hans Johnson, Stuart Wallace, Randy Gollub, Vince Calhoun, Kelvin Lim. White Matter Abnormalities in Schizophrenia: A Multi-site diffusion tensor imaging study. (Oral presentation at the Winter Workshop on Schizophrenia and Bipolar Disorder, February 2008, Montreux, Switzerland). 
Project 2: FIRST Program
Investigator: S. Chuck Schulz, M.D., John Lauriello, M.D., Oliver Freudenriech, M.D.
Period of Report: 4/1/07- 3/31/08

Introduction:

The Global Burden of Disease study found mental disorders to be the world's leading cause of disability; as four out of the ten diseases with the highest burden are psychiatric. About $25 \%$ of all individuals develop one or more psychiatric and behavioral disorders during their lifetime. Mental and emotional problems are often like physical problems; the sooner they receive treatment, the better the outcome. Even a person's response to antipsychotic medication improves with early intervention. The longer an illness is left untreated, the greater the disruption to the person's ability to study, work, make friends and interact comfortably with others.

Schizophrenia is an enigmatic illness that affects $1 \%$ of the world's population. Its severity is well known - both in terms of mortality and morbidity. For example, $10 \%$ of patients diagnosed with schizophrenia will end their own lives - surprisingly, mostly within the first five years of the initial diagnosis.

Furthermore, the lifespan of people with schizophrenia is at least eight years shorter than people without the illness. Morbidity has been measured by decreased quality of life, inability to obtain and retain employment, and the discomfort of going through a psychotic illness. Beyond this, there is an enormous impact on the families of patients who suffer from schizophrenia.

The FIRST clinics are designed to provide psychiatric evaluation for patients at the initial stages of a psychotic illness. Thus, the founding of these clinics will fulfill a major public health need - the provision of expert evaluation of the first episode of psychotic illness. These clinics that will be housed at the UNM, the UMN, and MGH will provide evaluation services for patients, their families, and referring clinics/practitioners. Recruitment and retention of patients suffering from schizophrenia has been noted to be one of the most challenging parts of learning more about this serious illness. Furthermore, by providing needed services at the outset of the illness, the retention of patients for longitudinal studies is substantially enhanced. This valuable service is being created to serve patients immediately and to provide a venue to apply research findings to improve care.

The overall goal of the program will be achieved through three separate platforms: the Glutamate Study, FIRST Clinics and the Spectroscopy/Technical Development Project. Below are the site reports from UMN, MGH and UNM on each of these aspects of the FIRST program.

\title{
Site Reports:
}

\section{University of Minnesota - S. Chuck Schulz, M.D., Kelvin Lim, M.D.}

\section{Glutamate Study}

\begin{abstract}
:
The glutamate study was designed to measure spectroscopic indices of glutamatergic metabolism and neuronal health early in the course of schizophrenia, and during the first year of standardized antipsychotic treatment. Individuals in the early stages of schizophrenia and healthy controls will be recruited at the three MIND sites to ensure an adequate, homogenous sample. We will use a novel technique of spectroscopy imaging at high-field (3T Siemens Trio at all sites) to study Glx (representing
\end{abstract}


primarily glutamate and glutamine) in multiple brain regions. We will concurrently acquire multi-spectral, high-resolution MRI to prospectively document expected brain volume reductions. Diffusion tensor imaging (DTI), to assess white matter tracts integrity, predicted to be affected by the glutamate-mediated cortical process, will also be acquired. Neuroimaging sessions will be repeated at 3,6 and 12 months. Repeated measures of symptomatology, cognition and social function will be assessed. Finally, saliva samples for analyses of single nucleotide polymorphism (SNP's) involved in glutamatergic neurotransmission, will be collected.

\section{Objectives and Findings:}

A great deal of discussion and planning has gone into the technical aspects of this study. For details, please see the accomplishments of the spectroscopy/technical development project listed below.

The glutamate application was submitted to the IRB in October and has been on hold pending approval from our General Clinical Research Center's (GCRC) Scientific Advisory Committee. On 1/15/2008, we received a letter from the GCRC outlining their concerns. We responded to the questions and concerns put forth by the GCRC Committee and received approval on $2 / 18 / 2008$. The study will be reviewed by the IRB on $2 / 27 / 2008$. We are in the process of submitting this study to the $3 \mathrm{~T}$ committee at the Center for Magnetic Resonance Research.

Regular conference calls with all three sites give us the opportunity to discuss IRB related issues. Now that we are getting closer to having IRB approval, the calls will be focused more on subject recruitment, enrollment, and testing.

Weekly lab meetings at the University of Minnesota site give us the opportunity to discuss issues related to the implementation of this study.

\section{Pending Grant Applications:}

American Foundation for Suicide Prevention. "Can Clinical Neuroscience Measures Predict High Risk for Suicidal Behavior in First Episode Schizophrenia?”

\section{Enhanced Clinical Services (FIRST Clinic)}

\section{Abstract:}

The overall goal of this research project is to establish a database of individuals in the early stages of schizophrenia. This database will allow us 1) to follow individuals who may be interested in participating in future research projects, and 2) collect and store clinical information for research purposes.

\section{Objectives and Findings:}

The IRB application was submitted in early October. We have been working closely with the IRB to restructure and clarify the purpose of this study. Our third submission was sent to the IRB last week and will be reviewed on $3 / 5 / 2008$.

Regular conference calls with all three sites give us the opportunity to discuss IRB related issues. Now that we are getting closer to having IRB approval, the calls will be focused more on subject recruitment, enrollment, and testing. 
Weekly lab meetings at the University of Minnesota site give us the opportunity to discuss issues related to the implementation of this study.

Dan Hanson, M.D., PhD. has been hired to head up the FIRST clinic. We are in the process of hiring a coordinator.

\section{Spectroscopy/Technical Development Project}

\section{Abstract:}

The purpose of the spectroscopy/technical development project is to develop the imaging and spectroscopy protocols for the glutamate project. This project also establishes the plan for data processing and addresses technical issues related to the glutamate project.

\section{Objectives and Findings:}

Regular weekly conference calls with all three sites help prepare us to carry out the glutamate study. These calls take place every Thursday at $11 \mathrm{am} \mathrm{CT}$.

Extensive discussions have taken place among the group to evaluate all of our options for data acquisition. We have decided on the PEPSI sequence for our spectroscopic acquisition.

Stephan Posse from New Mexico traveled to Minnesota and MGH to install the PEPSI software and train staff on data acquisition.

The imaging protocol was set for the anatomical, resting fMRI, DTI, oddball, and spectroscopy sequences.

An imaging repository/cataloguing system was put in place at the MIND Institute for purposes of organizing, storing, and archiving the imaging data.

Pipelines have been planned for the automated processing of imaging data.

Spectroscopy data from Minnesota has been analyzed using algorithms and processes at the MIND Institute.

An individual is being identified to take over the routine MRS processing. Hongji will train this individual. 


\section{Massachuttes General Hospital - Oliver Freudenreich, M.D., Don Goff, M.D.,Corinne Cather, M.D.}

\section{Glutamate Study}

\section{Abstract:}

Despite effective symptom reduction with antipsychotic medications, the majority of schizophrenia patients fail to achieve premorbid status and functional outcomes are generally poor. $\mathrm{N}$-acetylaspartate (NAA), a marker of neuronal viability, is related to cognitive function and may predict functional outcome. NAA is reduced in chronically-treated schizophrenia, but may be normal early in the disease. The clinical significance and mechanisms accounting for this NAA reduction are poorly understood. A second line of evidence suggests that glutamine (Gln) may be elevated early in the illness. Glutamine concentrations primarily reflect glutamate (Glu) cleared from the synapse, providing an in-vivo index of glutamatergic neurotransmission. It has been postulated that in schizophrenia there is a progressive excitotoxic process involving hyperglutamatergia, which disrupts neuronal function and accounts for the global cognitive defects and poor psychosocial outcomes of the illness, despite the beneficial symptom reducing effects of antipsychotic medication. The proposed project will use high-field (3T) 1H-MRS to study NAA, Gln and Glu in antipsychotic-naïve and minimally-treated schizophrenia. These neurometabolites will be measured in multiple brain regions known to be affected in schizophrenia. $1 \mathrm{H}$ MRS assessments will be repeated at 1, 3, 6 and 12 months. Treatment with antipsychotic medications will be standardized and symptoms, side-effects, cognitive and social function will be assessed prospectively. A group of healthy volunteers will also be followed longitudinally.

Our overall hypothesis is that early in schizophrenia there is an evolving excitotoxic glutamatergic process which disrupts neuronal function in several distributed brain networks and accounts for the cortical atrophy, cognitive defects and poor psychosocial outcomes of the illness, despite the beneficial symptom reducing effects of antipsychotic medication.

Related to the specific study of glutamate and its hypothesized impact on brain imaging and neuropsychological parameters is the hypothesis that imaging and neuropsychological measures can be useful in clarifying psychiatric diagnosis and prognosis. In translating this research project to the clinical arena, the data from brain imaging and neuropsychology will be analyzed to assess the utility of these measures to assist in diagnostic certainty. In addition, some studies have found that brain imaging measures are related to medication response. Therefore, imaging and neuropsychological testing will be correlated with symptomatic outcome at follow-up visits. We focus on the following specific aims:

\section{Objectives and Findings:}

1. Examine the baseline glutamate profile and its relationships to neuronal viability (NAA) and volumetric measures in various gray matter $(\mathrm{GM})$ cortical regions in minimally-treated SZ subjects early in the illness.

2. Examine the temporal change of glutamate neurochemistry, NAA and cortical volume over the first year of treatment in SZ.

3. Brain imaging, neuropsychological testing, and clinical information will be used to enhance psychiatric diagnosis and response to medication treatment. The relationship of these parameters will also be correlated to cognitive and functional outcomes. 
4. To create cell lines for future genetic testing in the SZ and control groups and to conduct a pilot examination of the relationship between genes related to glutamatergic neurotransmission and early brain changes and clinical deterioration in SZ.

Accomplishments to date:

IRB Submission: We submitted our IRB proposal to the Partners IRB on December 12, 2007. On $1 / 24 / 08$, we received a request from the Partners IRB to submit detailed drug information for every antipsychotic in the specified treatment algorithm. These forms were submitted on 1/29/08 and the proposal is pending approval. We anticipate review by March 1, 2008.

Subject Recruitment: Once we receive IRB approval, we are ready to begin enrolling patients in the protocol immediately. We anticipate enrolling our first patient by March 15, 2008 and do not anticipate having any difficulty enrolling 3-4 patients per month in the first 2 months from approval. Thereafter, we will aim to enroll 2 patients per month, permitting us to complete our patient recruitment goal of 15 patients by September 15, 2008.

Pending Grant: In collaboration with other members of the MIND consortium, we submitted a linked application to the American Foundation for Suicide Prevention. If awarded, the grant would provide an additional $\$ 75 \mathrm{~K}$ of funding over a 2 year period and would add an investigation of the longitudinal course of suicidal thoughts and behavior in our minimally treated first episode patients.

\section{Enhanced Clinical Services (FIRST Clinic)}

\section{Abstract:}

Pharmacologic and psychosocial intervention at the first episode of psychosis is considered critically important in terms of reducing duration of untreated psychosis (DUP), since delays in treatment have been linked to poorer outcomes. Evidence is accumulating that optimal treatment of first episode psychosis requires specialized, multi-component recovery-oriented programs comprised of low dose antipsychotic medication combined with cognitive behavioral therapy (CBT).

\section{Objectives and Findings:}

In expanding the MGH First Episode and Early Psychosis program, we focus on the following specific aims:

1. Provide clinical and diagnostic psychiatry services to individuals with early episode psychosis. Diagnostic assessment includes medical evaluation, laboratory tests, genetics, neuroimaging, cognitive testing, and structured clinical assessments culminating in a comprehensive consultation and, when appropriate, treatment

2. Provide individual and group cognitive-behavioral therapy to first episode patients to improve medication adherence, coping skills, and to educate them about the illness with the goal of affecting long-term outcomes

3. Promote wellness behaviors and minimize adverse health effects from medications early in the course of the illness

4. Provide family education about psychosis 
Accomplishments to date:

Clinic Volume: The FIRST clinic is well established as a state-of-the-art First Episode program, offering enhanced clinical outpatient services for patients who are either early in the course of psychosis or who have recently developed psychosis for the first time. MIND funding has facilitated the delivery of enhanced clinical services for all of our patients and has permitted us to provide free care to patients whose insurance is not accepted by MGH since 7/1/07. Since 7/1/07, the First Episode and Early Psychosis Program at MGH has seen 33 new patients for consultation/second opinion, ongoing medication management, individual cognitive behavioral therapy (CBT) and/or family psychoeducation. In early March 2008, we will begin our first weekly CBT group for individuals with first episode psychosis. In order to lay the groundwork for this group, we hosted a seminar for 12 of our first episode patients and paid two peer educators, Brooke Katz, RN and Lisa Halpern, M.A., to speak to the attendees to talk about their personal experience of living with and recovering from psychotic disorder. The program was very well received and following the program, all of the 12 attendees (and three other individuals who were not able to attend due to prior commitments) expressed interest in joining a weekly CBT group.

Diagnostic Evaluation and Family Feedback Session: The initial visit is structured as a single half-day visit for patients and parents to the MGH. Both the patient and family meet for one hour with a psychiatrist and for one hour with a cognitive behavioral psychologist. The majority of each of these meetings is focused on meeting individually with the client, although each meeting starts with a brief meeting with the family to give the opportunity for them to voice their observations and concerns. Following these diagnostic interviews, which involve a thorough review of personal and family history, review of previous treatment records and tests, and clinical interview; the program staff convene to discuss the case and formulate treatment recommendations. Depending on the needs, patients and families are offered clinical care that includes psychopharmacological and psychological treatments. Ancillary rehabilitation services are accessed early, and depending on the individual case, referrals are made to various Massachusetts Department of Mental Health services. To determine the best course of action for each patient, the program staff meet weekly to review clinical questions and to discuss new research pertinent to clinical care. In addition to attending to psychiatric needs, focus is placed on the prevention of medical morbidity and the staff work closely with primary care doctors, weight control specialists and smoking cessation programs.

Referral Sources and Program Development: The presence of the First Episode and Early Psychosis Program, located in the Wang Building on the main campus of the Massachusetts General Hospital, continues to be a valuable asset, providing a link to other specialists and primary care physicians. Good working relationships among clinicians and continuity of care are fostered within the MGH by formal and informal consultation and by smooth integration outpatient care for patients scheduled for discharge from the MGH Psychiatric Inpatient Service, and from the MGH Acute Psychiatry Service (APS), the psychiatric emergency room with the highest volume in the Northeast. We strive to see new patients within seven days of hospital discharge and to offer formal consultations to other MGH physicianreferred patients within two weeks. In addition to strengthening ties to the APS, a close collaboration has been developed with a similar service at McLean Hospital, Belmont, Massachusetts, by having a psychiatrist (Dr. Holt) available by page 24 hours/day to screen possible referrals and schedule initial appointments. To further these referral relationships, a member of the clinical team has lectured every three months in the APS to the residents, to educate them about the importance of early detection of psychosis in light of recent research findings, and to inform them of the criteria and referral procedure for the FEPP clinic. Meetings are also held on an ongoing basis with the psychiatrists-in-charge and the directors of the Psychotic Disorders Unit, the Short Term Unit, and the Psychotic Disorders Partial 
Hospitalization Program at McLean Hospital to facilitate referrals for patients who are currently hospitalized for their first psychotic episode and are in need of ongoing outpatient psychiatric care.

Hiring: We have not been able to achieve our goal of hiring social work support due to administrative issues at our site which have prevented these salary lines to be available to us. To date, we have compensated for this by embedding case management into cognitive behavioral therapy and family psychoeducation. We have strenuously worked to remove this impediment to hiring; the timing of the resolution will possibly affect whether we are able to hire social work staff in the coming months.

IRB application: See attached. We submitted our IRB proposal to the Partners IRB on December 12, 2007. On $1 / 24 / 08$, we received a request from the Partners IRB to submit detailed drug information for every antipsychotic in the specified treatment algorithm. These forms were submitted on 1/29/08 and the proposal is pending approval. We anticipate review by March 1, 2008.

\section{Summary}

We have exceeded our goal of seeing a minimum of 3 first episode patients per month. Our clinic volume is a direct result of our close collaboration with the psychiatric inpatient unit at MGH and the MGH psychiatric emergency room as well as our close collaboration with McLean Hospital. We have standardized our diagnostic evaluation and family feedback session; spending approximately 3 hours with patients and families in the initial visit. We have been unable to meet our goal of hiring social work staff due to administrative issues that have prevented the release of funds to our site.

We continue to believe that our flexible, team-based outpatient approach for patients with first episode psychosis can lead to good long-term psychosocial adjustment. We are currently tracking long-term outcomes more formally. We continue to see a subset of patients who attain full remission of symptoms and return to previous level of function at work or in school.

\section{University of New Mexico - John Lauriello, M.D., Juan Bustillo, M.D.}

\section{Glutamate Study}

\section{Abstract:}

Despite effective symptom reduction with antipsychotic medications, the majority of schizophrenia (SZ) patients fail to regain premorbid status and functional outcomes are generally poor. Our overall hypothesis in this project is that early in schizophrenia there is an evolving excitotoxic glutamatergic process that disrupts neuronal function in several distributed brain networks and accounts for the cortical atrophy, cognitive defects and poor psychosocial outcomes of the illness, despite the beneficial symptom reducing effects of antipsychotic medication.

\section{Objectives and Findings:}

During the funding period, the UNM research team contributed greatly to the start-up of the FIRST Project and all subprojects. Specific contributions are outlined below:

At the start of working on this project, the UNM team, Dr.Bustillo in particular, was responsible for writing the first draft of the Glutamate protocol. Subsequent revisions were made by the team and the final protocol template was distributed to all sites. 
As with the other subprojects, the UNM team brainstormed study design and how each of the subprojects would interface. We proposed the initial measure selection and developed a detailed study schedule to delineate what would happen at each study visit, and collaborated with other study sites to refine the measures and procedures. We then compiled, formatted, and distributed measures to all sites and assisted Jody Roberts with creating research binders.

The UNM team wrote the consent template that was distributed to all study sites and again collaborated with Jody Roberts on the marketing materials design and content.

The UNM team submitted the Glutamate Project IRB application and all necessary documentation and attachments. It has received departmental approval and is currently pending IRB approval.

As part of this subproject and the overarching FIRST protocol, the UNM team worked collaboratively with the other sites on a joint submission of a Suicide Prevention grant proposal (see Appendix A). This involved working under a tight deadline, compiling and formatting additional measures for distribution, revising the study protocols, consents, and schedules, generating additional budgets and more. This grant application if pending approval.

Obstacles have included difficulties developing a workable MRS protocol that interfaces well with other study subprojects. See below for additional details.

\section{Pending Grant Applications:}

"Can Clinical Neuroscience Measures Predict High Risk for Suicidal behavior in First Episode Schizophrenia?" American Foundation for Suicide Prevention

\section{Enhanced Clinical Services (FIRST Clinic)}

\section{Abstract:}

The overall goal of the FIRST Project is to establish and manage three FIRST Clinics, which will provide a comprehensive diagnosis of patients during their first episode of psychosis, and to conduct research on the early diagnosis and linkage to treatment of schizophrenia. Other goals specific to UNM include: 1) collecting diagnostic and clinical data to be able to provide referring physicians with a comprehensive report; 2) collecting basic descriptive statistics for program evaluation purposes; and 3) providing supportive services and education to patients and families during this difficult time of early diagnosis.

\section{Objectives and Findings:}

Over the course of the last year, the UNM team (Drs. Lauriello and Bustillo, Tara Biehl and Heather Hawk) have participated in FIRST conference calls on a biweekly basis in order to discuss new and ongoing issues to move the project forward. When Jody Roberts was away on maternity leave, the UNM team assumed responsibility for program management in her absence. This included coordinating and documenting FIRST conference calls. During this time period, the UNM team also worked closely with coordinators from other sites during RFP submission to MIND and helped with budget coordination and completion. 
The UNM team wrote the FIRST Project protocol that was later distributed to all study sites and refined. We initially brainstormed as a group the study design and how each of the subprojects would interface.

The UNM team wrote the consent template and generated a detailed study schedule to delineate what would happen at each study visit. We assisted in selecting and refining measures, then compiled, formatted, and helped distribute measures to all study sites for consistency and ease of data entry.

The UNM team met with Jody Roberts to help develop a marketing approach and contributed to marketing materials design and content.

The UNM team collaborated with the other sites to brainstorm and research enhanced services components on the biweekly conference calls, via email and through direct contact between site research coordinators. Recently, the UNM team attended a seminar regarding organizing and facilitating multifamily, psychoeducational groups in anticipation of incorporating this into the enhanced services portion of the FIRST Project.

The UNM team submitted the FIRST Project IRB application and all necessary documentation and attachments. It has received departmental approval and is currently pending IRB approval.

Obstacles have been starting fresh and building a new clinical research program from the ground floor. Other obstacles have included developing an effective outreach program that will extend state-wide, while being able to provide consistent and quality treatment options.

\title{
Spectroscopy/Technical Development Project
}

\begin{abstract}
:
Glutamate is the major excitatory neurotransmitter in the mammalian brain, and it has been proposed to play a role in schizophrenia, pain conditions, epilepsy and neurodegenerative disorders (Mattson 2003, Mullins 2005). It is not surprising then that considerable thought and energy has gone into finding ways to reliably measure neural glutamate (Glu) and glutamine (Gln) levels in both normal and psychiatric subject groups. The use of higher field strengths such as $4 \mathrm{~T}$ and $7 \mathrm{~T}$ with greater spectral resolution has led to higher selectivity and accuracy allowing Glu and Gln levels to be detected separately (Mullins 2005). However, high field systems are currently not readily available or appropriate for clinical use. In this application we propose to address the ability of commercially available 3T MR imaging platforms to detect, encode, and quantify metabolite signals that are particularly challenging with conventional chemical shift spectroscopy. Ultimately the techniques implemented in this project will be applied to several disease states where changes in glutamate may play a major role, and will be immediately applicable for use in the Glutamate subproject. In addition these techniques can be optimized for other neurometabolites of interest furthering the overarching goal of the MIND institute by providing new tools for study, diagnosis and monitoring of treatment in mental illness and neuroscience discovery.
\end{abstract}




\section{Objectives and Findings:}

Over the course of the last year, the UNM team (Drs Bustillo, Lauriello and Posse) collaborated with MIND scientists (Dr Mullins - the PI for this Spectroscopy/Technical Development Project - and Chen) as well as collaborators from UMN (Lim) and MGH (Jenkins) to implement and refine proton magnetic resonance spectroscopy (H-MRS) tools to use in the study "Glutamate dynamics in early schizophrenia and clinical outcome." The goal has been to measure glutamate and glutamine, in addition to the traditional metabolites (NAA, Cre and Cho) in several brain regions, in clinically feasible time periods. We have been having regular weekly or bi-weekly conference calls to coordinate efforts towards this common goal.

Mullins and Chen implemented a time-to-echo (TE) averaged single voxel PRESS (10 min per subject) approach during the initial 6 months of 2007. This resulted in excellent fits for glutamate $(\mathrm{CRLB}<15)$ for a $12 \mathrm{cc}$ voxel in the dorsal cingulate cortex in several normal volunteers. Fits were also excellent for NAA, Cre and Cho $(<10)$, but suboptimal for glutamine $(>20)$.

Because of preliminary data from the UNM site suggestive of a robust correlation between cognition and glutamate+glutamine in schizophrenia $(\mathrm{n}=30)$ using PEPSI short TE data, the group decided to pursue this technique and implement it in the 3T scanners at UNM, UMN and MGH. Collaborator Posse has traveled to $\mathrm{MN}$ and Boston to assist with the implementation of the PEPSI sequence.

Four normal controls have been scanned at UNM with consistent glutamate+glutamine fits $<20$, across more than $1301 \mathrm{cc}$ voxels per subject. NAA, Cre, Cho and Ins also had excellent fits. Acquisition time was under 10 minutes. One subject has been scanned at UMN and one at MGH.

Obstacles have been the departure of Dr. Mullins from MIND and limited availability of collaborator Chen since then. We hope to overcome this limitation by having Drs. Calhoun and Posse lead the image analyses pipeline and hope to identify a MIND employee who can be trained to implement these analyses.

\section{Conclusion:}

All three sites anticipate IRB approval in early March. Community outreach and program awareness has been emphasized over the past few months to improve subject recruitment once approval is received. The group continues to meet via teleconference on a weekly basis to discuss and review any imaging related issues, along with bi-weekly calls for protocol and subject related topics. We plan to continue these calls for the duration of the project to ensure consistency and reliability in the treatment and data acquisition. There is a data plan in place at MRN to facilitate on-going analysis during the longitudinal study. All site PI's are committed to continuing their collaboration for future research projects by obtaining external grant funding. 


\title{
Project 3: MEG Consortium \\ Investigator: Michael P. Weisend, Ph.D., Matti S. Hämäläinen, Ph.D. Period of Report: 4/1/07-3/31/08
}

\begin{abstract}
:
Magnetoencephalography (MEG), a non-invasive functional neuroimaging technique with high spatial and temporal resolution, is available at over 100 sites worldwide. In combination with magnetic resonance imaging (MRI), MEG can provide a record of where and when, on a scale of milliseconds, activity is occurring in the brain. Large-scale studies that detail the neurophysiological and neuroanatomical differences between patient populations and healthy control subjects can substantially advance our understanding of the neural bases of mental illness and neurological disease. Studies of large patient populations often require the cooperation of multiple centers. Direct comparisons and pooling of data across MEG sites have been prohibitively difficult as a result of differences in hardware, software, and other site-specific factors. As part of the MIND DOE funding of the MEG Technology Program standardized procedures were developed for the purpose of collecting a dataset to be used to pioneer methods for pooling MEG data across The MIND Institute and its partner sites (Veterans Administration Minneapolis Center/Minnesota, Martinos Biomedical Imaging Center at Massachusetts General Hospital/Boston, and Los Alamos National Laboratory/New Mexico). The standardized data collection procedures were implemented and used to collect MEG data from 8 volunteer subjects ( 3 females, 5 males, ages 24-44, ) who traveled to the MIND partners MEG sites. MEG data were collected on two consecutive days using a 306-channel VectorView system (Elekta-Neuromag Oy, Helsinki, Finland) in Boston, a 248-channel Magnes 3600 WH system (4D Neuroimaging, San Diego, CA, USA) in Minneapolis, and a 275-channel Omega275 system (VSM MedTech, Coquitlam, BC, Canada) in Albuquerque. The current design using the same subjects at multiple MEG sites with multiple collections on each MEG instrument will allow us to compare variability within and across sites. MIND Institute scientists in collaboration with scientists at MGH, UMN, and LANL used this dataset to develop methods by which MEG data can be converted to a common format and analyzed through two data processing streams; MNE/Freesurfer which was developed at MGH and Brainstorm/Brainsuite which was developed in a collaboration of USC and LANL. The goal of this project is to standardize procedures for data collection and analysis across the Mind partner sites to make collaborations between investigators routine, not exceptional. Further, the software developments and data set arising from the MEG Technology program will be disseminated to MEG scientists worldwide.
\end{abstract}

\section{Objectives and Findings:}

Previous DOE funding has been applied to the standardizing and enhancing the capabilities of the MEG programs at MIND and the partner sites. Through meetings, phone conferences, and site visits we have standardized data collection procedures, stimulus delivery equipment, and data analysis methods. The bulk of this work was accomplished as part of the DOE funded, MEG Technology Project. The scientists at MGH, LANL, UMN, and MIND have developed methods by which MEG data can be converted to a common format and analyzed through two data processing streams: MNE developed at MGH and Brainstorm developed in a collaboration between USC and LANL. These software packages are freely available on the web sites of the Martinos Center at MGH and USC and have been downloaded by hundreds of researchers worldwide.

The project specifically targeted the MIND partner sites containing the three major instruments used world-wide: the Magnes MEG system at the University of Minnesota / VA Hospital, the Vectorview MEG system at the Massachusetts General Hospital, and the Omega MEG system at the Mind Institute in New Mexico. The project addressed the obstacles to cross site pooling of data by holding constant the subject population, stimulus presentation paradigms, and stimulus presentation equipment across three 
different sites with different MEG arrays. Holding constant the subject and stimulus parameters enabled focus on the MEG hardware and software issues. The MEG systems from different manufacturers have different numbers of sensors, different types of sensors, different sensor positions. In addition, they are operated in under different enviromental noise conditios and in different magnetically shielded rooms. The systems employ different methods of noise cancellation, mechanisms of head localization, filtering parameters, source localization parameters, and coordinate systems for specifying the sensor and source locations. At the outset of this project the software tools necessary to surmount the obstacles to pooling MEG did not exist. The tools necessary for unified analysis of the MEG data were developed. These tools include: (i) Software to convert the raw MEG data into a common file format (Functional Image File Format, FIFF), (ii) A Matlab toolbox for reading and writing MEG data files, (iii) Routines to take into account the different software noise compensation schemes employed in forward modeling, and (iv) A unified framework for the definition of sensor array geometries.

Preliminary analyses of these data have appeared in published reports. The results of one subject are presented in Fig. 1. Estimates of brain activity at $20 \mathrm{~ms}$ after median nerve stimulation show very good test/retest results within a single subject across instruments from different manufacturers and different software packages.

The objectives for the funding period were (1) to continue the methodological developments and (2) complete the analysis of the human data.

Methodological developments: The methodological developments targeted for the previous finding period were to provide tools for the interoperability between the two analysis streams to exchange and compare geometrical information derived from anatomical MRI and coordinate system alignment information and to develop metrics to quantify differences across data sets both in the sensor space and between source estimates.

We completed the database of the MEG consortium data. This database contains: (1) The continuous raw data acquired; (2) Event-related averaged computed with appropriate artifact rejection and filtering parameters; (4) Complete FreeSurfer reconstructions of the MRI data for boundary-element forward modeling and for the computation of corticallyconstrained source estimates; (5) MEG-MRI coordinate system alignment data; (6) The forward solutions and inverse operators for computing cortically-constrained minimum-norm solutions and statistical parametric maps. Identical copies of the database are maintained at MGH and MIND. In addition, the BrainStorm developers at USC have access to the database through the MGH site. The methodological developments of this project have had a significant impact on the MEG community at large. Since March 2007, the binary code of the C-based tools and the source code of the Matlab toolbox comprising the MNE software developed at MGH have been distributed through our website. In addition to the local users at the MGH Martinos Center and affiliated academic

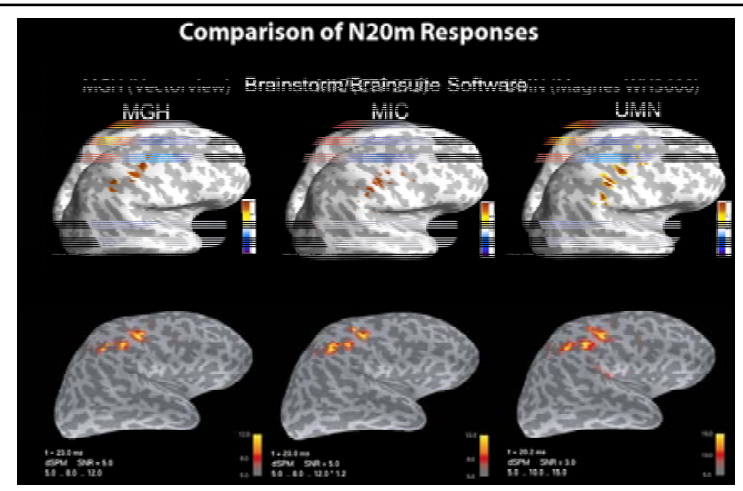

Figure 1: SPM localizations of activity from stimulatin of the median nerve. The top row shows the results of the Brainstorm/Brainsuite analysis pathway. The bottom row the MNE/Freesurfer results are illustrated in the bottom row. The left column shows the results from the MGH site, the middle column from the Mind and the right column from the University of Minnesota. institutions, 250 external users were registered to download the software by the beginning of January 2008. The software is supported by a 300-page manua. In collaboration with the FreeSurfer development team we are in the process of creating the associated wiki pages. In addition, we have organized MNE 
software training sessions both locally at the Martinos Center and at remote locations (University of Cambridge, University of Oxford, the MIND Institute, and University of Texas Houston). In, addition, MNE training is included in the curriculum of the Two-Week Multi-Modality Short Course organized annually at the Martinos Center. Discussion of the software features and reporting of bugs is promoted by mailing lists hosted at the Martinos Center.

The first version of BrainStorm was released in 2000. The major new release of BrainStorm, released in Spring 2004, includes tighter control of graphical interfaces and better integration of the workflow. Over 2,600 users have now re-registered and downloaded this new version. The current version of BrainStorm includes detailed help and tutorial pages and a forum for open discussion, questions and bug tracking. The software and documentation are distributed from a USC website. Each night, the SVN server packages the most recent version of the BrainStorm m-files into a zip-file and uploads it to the website, so that bug fixes and new functions are immediately available to user.

The methodological developments have also resulted in an NIH R01 proposal "Tools for Large-Scale Platform-Independent MEG Data Analysis" in which Dr. Hämäläinen from the MGH Martinos Center is the PI with subcontracts to LANL (Dr. Mosher) and USC (Dr. Leahy). This project will develop a standard work flow for the analysis of MEG data, coupled with specifications of data structures to enable cross site data sharing and comparison of results. In the project we will pursue four specific aims: (1) We will establish a unified workflow, data structures, and file formats for BrainStorm and MNE to enable sharing of data at all levels of analysis between the two packages. (2) Using available scripting languages, we will develop an efficient mechanism for analyzing multiple data sets with an emphasis on computing individual and group statistics with thresholding to correct for multiple comparisons. (3) Using the MIND MEG Consortium human calibration study data we will establish standard procedures for verification and validation of the implementations of MEG inverse procedures. (4) We will distribute open source software through linked sites at MGH and USC, with associated documentation, discussion forums, and online tutorials.

Analysis of Human Data: During the funding period, the MGH site has analyzed in detail the sources of variability in MEG signals. Specifically, we investigated somatosensory MEG data collected on the three sites and applied variance component analysis and nonparametric KL divergence analysis in order to characterize the sources of variability. The two analysis methods established that we can directly compare deflection peak time across systems and visits. Our analysis showed that inter-subject differences are the biggest factor in the signal variability. We demonstrated that the timing of the deflections is very consistent in the early somatosensory response, which justifies a direct comparison of deflection peak times acquired from different visits, subjects, and systems. Compared with deflection peak times, deflection magnitudes have larger variation across sites; modeling of this variability is necessary for data pooling. Some of the data suggest that the site effect may originate from subject physiological variability. We employed a random sampling approach illustrated that the timing of deflections is highly consistent even at the single-trial level. Hence, average across a large number of trials is not always necessary. Histograms built upon averages of a small number of single trials can better capture the inter-subject differences. The increased sensitivity of such approach can be helpful in studying differences in MEG responses between normal subjects and clinical populations.

The spatial analysis of the human data gathered as part of this study is at this time incomplete. This partly due to the fact that the efforts of $\mathrm{M}$. Weisend were diverted from this project to other funded projects. Notwithstanding this difficulty we revised and completed the manuscript on the analysis of the somatosensory data [1].

\section{Summary}

The long-term goals of this project are largely complete. System and site-independent tools form MEG data analysis are in place and functioning. This will allow data sharing with common data analysis 
techniques that has been impractical in the past. Further, the preliminary work has spawned an NIH R01 application for funding to continue the development and integration of these methods. Moreover, enduring collaborations between the MRN and MGH site have been established. There are additional two joint manuscripts currently in preparation using data form the MEG technology project.

\section{Manuscripts in Progress:}

M.P. Weisend, F.M. Hanlon, S.P. Ahlfors, J.C. Mosher, M.S. Hamalainen, and C.J. Aine (in preparation) "Source localization of Evoked and Induced Activity using a Minimum Norm Estimate"

M.P. Weisend, F.M. Hanlon, S.P. Ahlfors, J.C. Mosher, M.S. Hamalainen, and C.J. Aine (In preparation) "Changes in Evoked and Induced Activity as a Result of Stimulus Repetition"

\section{Presentations and Posters:}

Weisend, MP, McIntire, FM., Kerwin, A., Ahlfors, SP., Mosher, JC., Hamalainen, MS. (2007) Neural plasticity in the visual system as illustrated in time-frequency analysis of MEG recordings. Proceedings of the 13th Annual Meeting of the Organization for Human Brain Mapping, Chicago, Il, June 10-14, 2007.

M Weisend*, F Hanlon, R Montano, C Donahue,S Ahlfors, A Leuthold, J Mosher, A Georgopoulos, M Hämäläinen, C Aine (2006) Paving the way for cross-site pooling of MEG data. Biomag 2006, Vancouver, BC, Canada. August 21-25, 2006

J Mosher*,M Hamalainen, S Ahlfors, C Aine, A Georgopoulos, A Leuthold, P Volegov, M Weisend Transfer function analysis of different MEG arrays using a phantom and pseudorandom noise. Biomag 2006, Vancouver, BC, Canada. August 21-25, 2006 


\section{Project 4: Neurodevelopment Research Program \\ Investigator: Yoshio Okada, Professor of Neurology, University of New Mexico School of Medicine, Director of the BRaIN Imaging Center \\ Period of Report: 4/1/07-3/31/08}

The Neurodevelopment Research Program was formed to guide the direction of MIND scientific initiatives concerned with our understanding of brain development. Although we are witnessing an enormous progress in the development of molecular biological tools, electrophysiology and optical recordings from sub cellular elements such as synaptic terminals, our understanding of human brain development is still poor and in its early stages.

One of the main goals of this Program is to develop new tools or refine existing tools. In addition to clinical MR scanners and a whole-head MEG system for adults and children, the MIND Institute has a one-of-kind prototype MEG system optimized for babies called babySQUID ${ }^{\circledR}$, developed by Yoshio Okada, Ph.D. MEG is a completely safe technique capable of providing novel information about electrophysiology of cortical neurons nearly in real time. The studies that went forward in 2007 under this Program, described below, specifically target neurodevelopment and brain injury during the perinatal period, just before or after birth.

Project 4.1: Neurodevelopment Research Program: Diagnosis of Epilepsy and Epileptogenesis After Brain Injury in infants

Investigator: Julia Stephen, Ph.D.

Co-investigators: John Phillips, M.D., Tongsheg Zhang, Ph.D., Ting-Ting Kou, Ph.D.

Period of Report: 4/1/07-3/31/08

\section{Abstract:}

Brain injuries during the perinatal period often lead to epilepsy. The epileptiform activity, if it goes untreated for a long time, lead to a generalized brain damage with often severe consequences on the intelligence and daily function of the affected infants. If epilepsy is detected early in life, say soon after birth, the generalized damage can be prevented with minimal consequences. Thus early diagnosis is again important. A key to the treatment of epilepsy is identification of brain regions that initiate epileptiform seizures. Epileptiform activity can be detected with clinical EEG. However, it is still difficult to identify the areas with interictal activity that eventually produce seizure. Recently we found that there are two classes of interictal spikes in infants with neocortical epilepsy. One subset is associated with high frequency signals (HFSs) in the range of 80-200 Hz, whereas the second subset does not exhibit this HFS. We hypothesize that this HFS is analogous to the so-called fast ripple found in adult patients with mesial temporal lobe epilepsy (TLE) and in animal models of this TLE. Engel and his colleagues believe that this fast ripple is the biomarker of epileptogenesis since it is found only in those areas of the limbic system where one finds electrographic ictal activity. In this study we tested the prediction if the HFS in our pediatric population is a biomarker of epileptogenesis, the areas with HFS eventually produces an electrographic seizure.

\section{Objectives and Findings:}

1. Test whether the HFSs associated with a subset of interictal spikes are produced by the brain areas that eventually produce electrographic seizure. Conversely, test the prediction that all the areas that produce electrographic seizure produces interictal spikes with HFS. We used the generator localization algorithms to identify the generators of the HFS and electrographic seizure and compare their locations. 
2. Study how the multiple seizure sites interact with each other using the Granger causality analysis (GCA). We expect that the connections among the seizure sites will become stronger with time (i.e. with age) or with the increase in severity of clinical seizures.

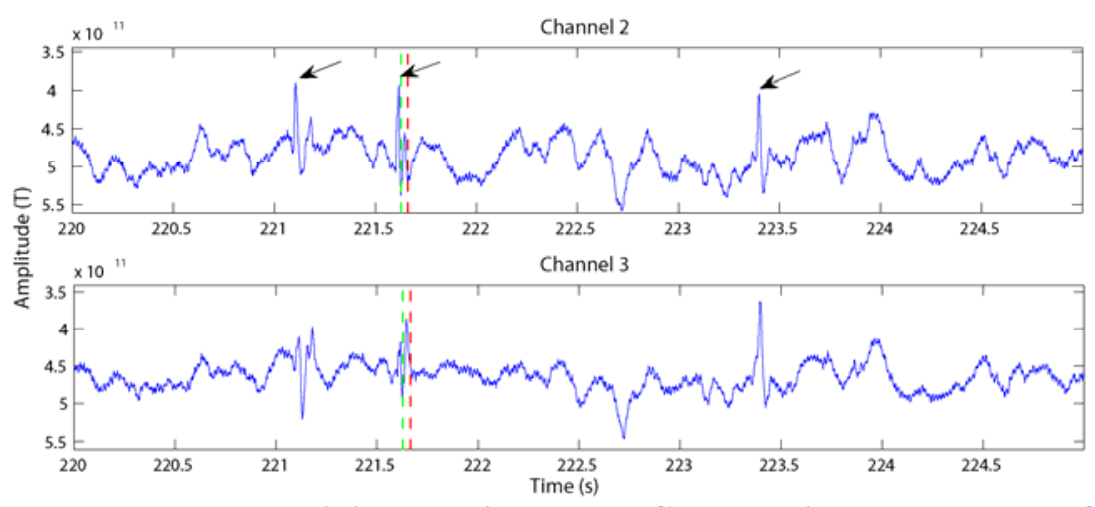

Figure 1: Abnormal sharp-wave activity seen in the MEG record in two channels. Only one of the three sharp-waves shown, demonstrated HFS (see green and red line).
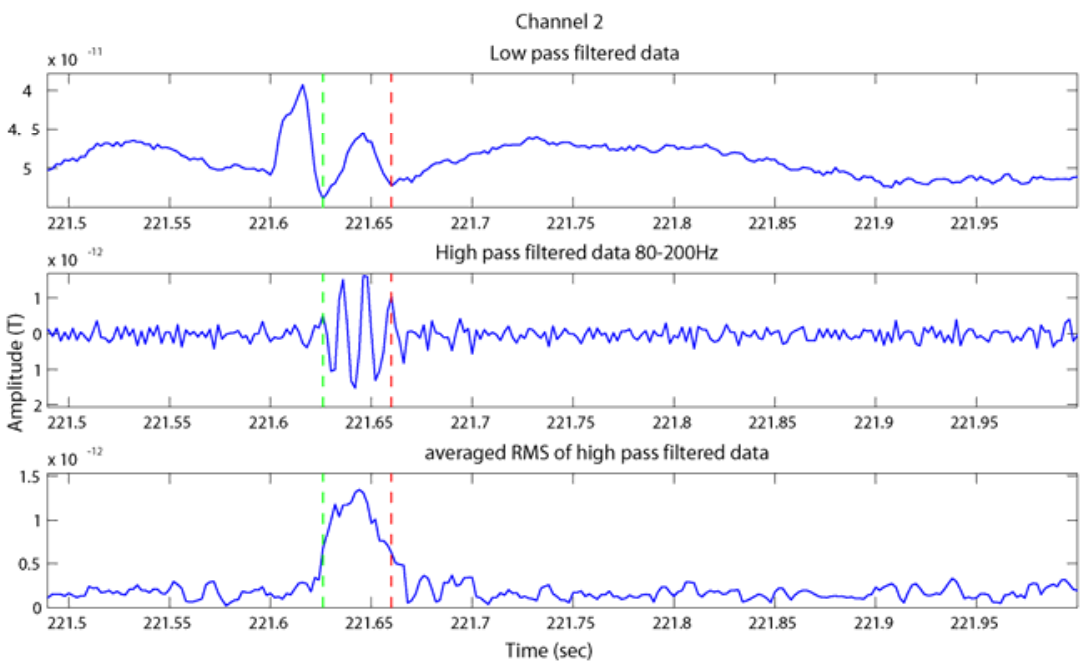

Figure 2: An example sharp-wave event that demonstrated high frequency activity in the same channel as shown in Error! Reference source not found..

Figure 1 shows a segment of spontaneous MEG signals measured with the babySQUID ${ }^{\circledR}$ from a 16month infant with epilepsy. The time traces are displayed for a set of channels over $5 \mathrm{~s}$. Note that interictal spikes are clearly seen in this record denoted by arrows. The signal amplitudes were as much as $10 \mathrm{pT}$.

The onset and offset of an epoch that met the criteria for HFS as described by Staba et al. 2003 is shown with a green and red line, respectively. The first row shows the wideband signal. The second row is the same record filtered with a bandpass filter having a passband of $80 \mathrm{~Hz}$ to $200 \mathrm{~Hz}$. The third record is the same filtered signal after rectification. Note that the HFS signal is present only for some of the interictal spikes even though their amplitudes are comparable. The fact that the HFS was only present during some of the interictal spikes and it did not always coincide with the spike peak indicate that it is not simply a high frequency component of the spike-wave complex. This HFS appears to be generated in the cortex since the HFS showed polarity reversal across spatially adjacent sensors of the babySQUID ${ }^{\circledR}$, strongly suggesting that it was generated in the cortex just below the polarity reversal line. 
Through the preliminary study of these identified HFOs we have identified a potential increase is frequency with age (our younger children showed oscillations $\sim 80 \mathrm{~Hz}$ whereas our 6 year old child showed oscillations in the $120 \mathrm{~Hz}$ range). This may prove to be a developmental difference in the frequency range of the HFO reported by Engel and colleagues. In addition, we localized the high frequency activity and found some correspondence between some of the interictal activity. This localization allowed us to show that we will be able quantitatively compare the locations of the high frequency activity relative to the lower frequency interictal spike activity to reliably test our hypothesis that these HFOs point to the area of epileptogenesis. We have submitted and received a fundable score on an NIH R21 grant, High frequency activity in infants with epilepsy, to further study these HFOs.

Project 4.2: Neurodevelopment Research Program: Multisensory Integration and Development of Mirror Neurons in Children with Autism: In Search of an Early Biological Marker with MEG Investigators: Julia Stephen, Andrew Mayer, Juliana Pare-Blagoev (MIND) Dina Hill, Brian Lopez (UNM)

Period of Report: 4/1/07-3/31/08

\section{Abstract:}

There is some evidence that children with autism may be abnormal in their development of ability to integrate sensory information across modalities (e.g. somatosensory and auditory) and in the development of a type of neurons called mirrors neurons. Mirror neurons were initially discovered in the primates. They are called mirror neurons because they behave as if they are mirroring movements of other primates. Current theories of autism implicate sensory atypicalities as common symptoms of autism and these atypicalities may have lasting effects on the individuals' development and behavioral presentation. Moreover, recent results show abnormal cortical connectivity in children diagnosed with autism, suggesting poor communication between cortical areas. It has been proposed that sensory integration will be impaired in autistics due to the requirement of cooperation between disparate brain regions, with some confirmation of this hypothesis showing that autistics respond differently to sounds with concurrent electrical stimulation to the wrist. A sensory integration paradigm is a bridge between simple sensory and complex cognitive tasks, requiring no response from the subject but invoking activation of a complex cortical network. As for mirror neurons, it has been recently suggested that they might be critical for understanding the emotional state of others and empathy. One of the defining characteristics of autism is emotional detachment. Williams et al. (2001) recently hypothesized that children with autism may have deficits with their mirror neuron system which does not allow them to imitate other's behaviors and therefore leads to difficulties with engaging with others as well as deficits in social communication. There is growing evidence of mirror neuron abnormalities in subjects with autism. Although much of the earlier work was performed on adults, a recent fMRI study on children (average age 12 years) has also shown decreased signal amplitude in the frontal region (the pars opercularis) known to contain mirror neurons. In this study they also showed a correlation between fMRI signal strength in this region and social functioning ability in the children with autism. Although there is some evidence for abnormality in sensory integration in children with autism, we still know very little about how the sensory integration is abnormal and about the development of this abnormality. Nor do we know the development of abnormality of functioning of the mirror neurons in these children. In this project we examined the abnormality in sensory integration and mirror neurons in a patient population using the babySQUID®.

\section{Objectives and Findings:}

To characterize the development of sensory integration in children with autism or more generally autism spectrum disorder (ASD) compared to neurotypical toddlers (3-6 years of age). In particular we studied 
the integration of auditory (A) and somatosensory (S) information in these children. Behavioral reports suggested that the integration response would be different in children with ASD. Our data found a significant developmental pattern where this multisensory integration response is longer in children aged 6-8 months ( $\sim 150 \mathrm{~ms})$ whereas the response in all children aged 8-42 months displayed a mean latency of around $75 \mathrm{~ms}$. These results suggest that the ASD children may show a delay in development (related to the intermediate latency of $\sim 100 \mathrm{~ms}$ ) or it could be different altogether. We have received pilot project money to continue this project from UNM HSC CTSC program. We also have two other external pending grant applications to perform follow-up studies in the ASD toddler population.

\section{New Technologies:}

Development of software associated with babySQUID ${ }^{\circledR}$ MEG data analysis has continued. We now have programs to ease the identification of sleep stage. A program to automate sleep stage characterization is under refinement. We have automated the signal averaging and onset detection of the multisensory processing response. We also improved on the analysis to allow us to use the BESA program for source localization. Additional improvements to allow us to use additional multisource analysis packages are ongoing.

\section{Manuscripts in Progress:}

Stephen, JM, Romero, L, Okada, Y. Development of auditory and somatosensory integration in infants and young children. To be submitted April 2008.

Stephen, JM, Romero, L. Phillips, J. Okada, Y. High frequency activity in infants with epilepsy. To be submitted May 2008.

\section{Presentations and Posters:}

Stephen, J. Functional Neuroimaging In Children: In Search Of An Early Marker For Autism. Invited Talk, Autism Symposium at the Southwest Conference on Developmental Disabilities, Albuquerque, NM Oct. 2007.

Julia M. Stephen ${ }^{1}$, Dina Hill ${ }^{2}$, Brian Lopez ${ }^{3}$, Lucinda Romero ${ }^{4}$, Yoshio Okada Exploring Brain Connectivity in Toddlers with Autism using the Pediatric MEG System called babySQUID Poster Presentation at Keystone Symposium: Towards Identifying the Pathophysiology of Autistic Syndromes. February 24-28, 2008, Santa Fe, NM. 
Individual Projects 


\title{
Project 5: Direct Detection of Neuronal Actity Using MRI \\ Investigator: Krastan Blagoev, Ph.D. (Charles Gasparovic, Ph.D, Co-PI) \\ Period of Report: 4/1/07-3/31/08
}

\begin{abstract}
:
An imaging modality with the tomographic precision of magnetic resonance imaging (MRI) and the high temporal resolution of electrophysiological techniques, such as electro- and magneto-encephalography (EEG/MEG), would be of great utility to neuroscience and medicine, allowing researchers and clinicians to map the changes in the neuronal dynamics associated with a given stimulus directly. The ability to localize both in space and time the neuronal response will revolutionize our understanding of brain function in health and disease. The advancement of the new technique proposed and demonstrated here will aid our understanding and treatment of all neurological disease as well as surgical planning, because it will be able to localize with great precision the lesion site and/or a specific neural function. Currently, the predominant MRI technique to study brain function, functional MRI (fMRI), provides only an indirect measure of the neuronal (electrophysiological) response and is limited in temporal resolution to several seconds. While electrophysiological techniques such as electro- and magneto-encephalography (EEG/MEG) have superb temporal resolution, the spatial localization of these techniques suffer from an ill-posed inverse problem. Event related neuronal current changes, will induce changes in the magnetization of the water in the brain that can be measured using MRI, leading to a noninvasive functional technique with high spatial and temporal resolution. To understand the effect stimulus induced changes in the neuronal currents in the human cortex, and to develop, optimize, and validate a new imaging technique based on this phenomena we will undertake the following specific aims.
\end{abstract}

\section{Objectives and Findings:}

To understand the effect stimulus induced changes in the neuronal currents in the human cortex, and to develop, optimize, and validate a new imaging technique based on this phenomena, we undertook to develop and optimize a magnetic resonance spectroscopy (MRS) based techniques for directly localizing electrophysiological neuronal activity. The signal dephasing effect we observe with MRS occurs within a few hundred milliseconds of the stimulus onset and is temporally consistent with the MEG signal generated by the same stimulus. Our hypothesis is that the mechanism underlying the MRS signal change is the synchronization of the neuronal population after stimulation and the consequent local change in current density and corresponding increase of magnetic field gradients. We propose to test this hypothesis by investigating neuronal current changes related to two different sensory stimuli (reversing checkerboard and median nerve stimulation) using

During the past funding period, we tested the feasibility of detecting the effect of event related neuronal currents (ERC) in the brain on the water proton magnetic resonance signal using single voxel proton magnetic resonance spectroscopy (1H-MRS). Our approach was motivated by the potential advantages of high signal-to-noise and time resolution inherent in single voxel 1H-MRS relative to echo-planar imaging methods. An extended series of experiments was initially conducted to optimize our protocol, exploring several variations on the visual stimulus delivery, data acquisition, and physiological noise monitoring. Using the optimized protocol, human subjects were then presented with a brief visual stimulus (reversing checkerboard) at 27-30 second intervals and a series of 1H-MRS signals were obtained from a single voxel placed in the primary visual cortex. The site for voxel placement was selected on a subject-tosubject basis using anatomical markers and functional MRI (FMRI). Explicitly timed stimulus presentation and long inter-trial intervals were used to disambiguate potential signal relative to both ERC and the expected hemodynamic response. We assessed relative changes in both phase and the rate of signal loss (dephasing) due to putative ERC and hemodynamic response effects. Results from the phase data were negative for both the ERC and hemodynamic signals. In contrast, dephasing results for the 
hemodynamic signal were significant at the individual level. Although the statistical analysis of individual trials on a subject-by-subject basis yielded a negative result for the detection of ERC, this may have been due to the large amount of noise observed within the individual trials. We therefore averaged the individual FIDs at each time point over the total number of trials to increase the $\mathrm{S} / \mathrm{N}$ ratio. The mean and 2 standard deviations, corresponding to a 95\% confidence interval, of the baseline FIDs were then calculated and compared to the remainder of the FIDs. This analysis revealed that the mean FID associated with the ERC (FID 1) and peak of the BOLD response (FIDs 2-3) were always higher than the baseline state (FIDs 7-9) for all three subjects. Specifically, this increase was higher than 2 standard deviations above the mean of the last 3 FIDs in the series. The main result of this study, nonetheless, is the lack of an unequivocal signature of neuronal currents on the dephasing or average phase of the $1 \mathrm{H}-$ MRS signal. A manuscript reporting these findings was submitted to a major neuroimaging journal and is currently under review. Further characterization and removal of the possible sources of physiologic noise and other sources of variance from data of this type will be necessary prior to the detection of ERC on an individual subject basis.

\section{Manuscripts in Progress:}

Detectability of Neuronal Currents in Human Brain with Magnetic Resonance Spectroscopy Krastan B. Blagoev, Alexandre R. Franco, Andrew R. Mayer, Paul Mullins, and Charles Gasparovic submitted to Neuroimage (2008) 


\title{
Project 6: Knowledge Environment \\ Investigator: $\mathrm{H}$. Jeremy Bockholt \\ Period of Report: 4/1/07-3/31/08
}

\begin{abstract}
:
The MIND Institute has developed a comprehensive, web-based solution for managing the mental health research data sources collected on several thousand research subjects who have participated in clinical neuroimaging projects over the past 5 years. This research effort will expand, enhance, and refine these tools, promote interoperability, and provide full support of genetic, MEG, EEG, physiology, and behavioral data sources. Finally, statistical tools will be integrated into our turnkey solution to provide a comprehensive quality assurance capability. The above effort will lead to the establishment of a biomedical knowledge environment that has been optimized for clinical neuroscience discoveries. The chief goals of this effort are to refine, enhance, and optimize the neuroinformatics software developed by the MIND Institute for data acquisition, archiving, querying, retrieval, integration and management of the clinical neuroscience research activities of the MIND Research Network with the ultimate goal of establishing an optimized biomedical knowledge environment that will lead to neuroscience research discoveries.
\end{abstract}

\section{Objectives and Findings:}

We have worked with our MIND investigators, partners, and collaborators this past year and have made significant progress. We have achieved optimization of a remote real-time high quality clinical data capture tool. This tool has been deployed to field in a pilot study for the FIRST program, and is being field tested in Dr. Kent Kiehl's adult psychopathy program. We collaborated with the BIRN project to extend and enhance the XCEDE XML schema, and have also worked with BIRN to integrate the CDISC schema into XCEDE. By supporting XCEDE from our remote clinical data capture tool, we are positioned to have a highly extensible appliance that can be deployed into any number of clinical research program settings. During the next few months, we will be further piloting the clinical data capture tool in a multi-site consortium study being conducted by BIRN.

We have established optimized methods for multi-institutional data exchange tools. As MIND launches the FIRST program, we have worked with FIRST investigators to provide refined tools for collaboration and exchange of data in a multi-site clinical research program. We will use the lessons learned from this project to provide continuous improvement and enhancements to future use of these comprehensive tools.

We have established optimized methods for data mining and reporting from heterogeneous data sources collected across multiple institutions. We have built a sophisticated query front end that is capable of intuitively finding and exporting data across institutions, studies, investigators, projects, etc.

The scope of this project was essentially to establish the necessary building blocks for establishing a neuroscience knowledge environment optimized for mental health and brain disorders research studies. We have established the user requirements, tools needed, and a timeline for establishing a pilot knowledge environment in neuroscience research at MIND. We will disseminate these findings in a planned manuscript. We have also submitted an R01 proposal, 1 R01 MH084048-01 entitled "The establishment of a novel biomedical knowledge environment optimized for neuroscience research".

Budget Variations: There was budget variation associated with the current project. The primary reason for this variation was that the PI of the project was tasked by the organization to develop externally funded programmatic research in neuroinformatics. This effort was very successful as the organization submitted one R01 proposal to NCRR, and one R03 proposals to NIBIB, and 1 R01 proposal to NIMH 
(mentioned above). Additional proposals (R01, and SBIR) or resubmissions of proposals under review are planned as a result of funding this pilot research.

\title{
Manuscripts in Progress:
}

Bockholt, H.J., Scully, M., Scott, A., Lane, S., Calhoun, V. A neuroinformatics framework for establishment of a biomedical knowledge environment. (In preparation for submission March 30, 2008 to Neuroinformatics).

Bockholt, H.J., Lane, S., Ling, J., Scully, M., Calhoun, V. A remote real-time high quality assessment data capture appliance for clinical neuroscience research. (In preparation for submission March 30, 2008 to Neuroinformatics).

\section{Project 7: Application of Diffusion Tensor Imaging to Characterize Subjects with Schizophrenia Investigator: Arvind Caprihan, Ph.D. Period of Report: 4/1/07-3/31/08}

\begin{abstract}
:
Fractional anisotropy (FA) images obtained from diffusion tensor imaging (DTI) experiments were used to distinguish subjects with schizophrenia from healthy controls. Principal component analysis (PCA) is often used to reduce the dimension of data before applying more sophisticated data analysis methods such as non-linear classification algorithms or independent component analysis. This practice is based on selecting components corresponding to the largest eigenvalues. If the ultimate goal is separation of data in two groups, then these set of components need not have the most discriminatory power. Mind investigators measured the distance between two such populations using Mahalanobis distance and chose the eigenvectors to maximize it, a modified PCA method, which is referred to as the discriminant PCA (DPCA). DPCA was applied to diffusion tensor based fractional anisotropy images to distinguish agematched schizophrenia subjects from healthy controls. The performance of the proposed method was evaluated by the one-leave-out method. It was shown that for this fractional anisotropy data-set, the classification error with 60 components was close to the minimum error and that the Mahalanobis distance was twice as large with DPCA than with PCA. Finally, by masking the discriminant function with the white matter tracts of the John Hopkins University atlas, the Mind team identified left superior longitudinal fasciculus as the tract which gave the least classification error. In addition, with six optimally chosen tracts the classification error was zero.
\end{abstract}

\section{Objectives and Findings:}

Neuroimaging studies typically collect data from different modalities, such as MRI, EEG, and MEG, with common parameters across subjects with the goal of extracting useful information from these large data sets. Even within one modality of MRI, one could collect T1 and diffusion tensor imaging (DTI) data for mapping anatomic structure, fMRI for functional imaging, perfusion maps, and spectroscopy for metabolite mapping.

This approach has led to renewed interest in multivariate statistical methods to identify patterns which characterize groups of subjects or alternatively to identify patterns which best distinguish different groups of subjects. The importance of performing joint analysis of multi-modal data was recently demonstrated by Calhoun (Co-Investigator) in order to study the functional dependence across modalities in a unified 
manner. Calhoun has emphasized independent component analysis as a method for extracting relevant maps, but more conventional multivariate methods and recent methods based on support-vector machines can also be used similarly. Software toolboxes are available for these various techniques.

One problem in handling large data sets is associated with the high dimensionality of the image based data. In a multi-modal imaging study one can easily generate more than $10^{6}$ voxels per experiment. In such cases principal component analysis (PCA) has been widely used for exploratory data analysis to reduce data dimensionality, before applying other methods such as independent component analysis or non-linear pattern recognition. If the goal of a given study is to discriminate between two or more groups, then applying standard PCA for feature reduction can undesirably eliminate features that discriminate and primarily keep features that best represent both groups. We study an alternative method for selecting features which maximizes the Mahalanobis distance between two groups. This method is not well known in the medical literature and has the interesting property that eigenvectors are the same as in conventional PCA but are ordered differently to maximize the Mahalanobis distance. An optimization step is not required as the optimal ordering of eigenvectors is known analytically. We call this method the discriminatory PCA (DPCA).

Schizophrenia is characterized by disruption of cognitive functions which among other things may be related to impairment of white matter integrity. DTI is a macroscopic measurement technique that allows the study of white matter microstructure based on anisotropic diffusion of water in white matter bundles. DTI measurements of fractional anisotropy, axial diffusivity, and radial diffusivity are considered to be measures of white matter fiber bundle coherence, organization and density. In addition, DTI based tractography can be used to study integrity of white matter fiber connections. There have been studies which have shown that fractional anisotropy (FA) measurements can identify white matter regions which distinguish schizophrenia subjects from healthy controls. These studies did not show how useful these markers were as predictive measures for distinguishing the two groups.

We applied DPCA to a fractional anisotropy (FA) data set from 45 schizophrenia subjects and 45 agematched healthy controls. We have shown that the proposed method can be important when large data sets from different modalities is collected and where it is important to retain group differences in the exploratory dimensionality reduction step. In our example data set, we were able to reduce the image dimension to 60 while maintaining classification performance comparable to that of the full data set and better than that of standard PCA data reduction. The left superior longitudinal fasciculus yielded the maximum discrimination between schizophrenia subjects and healthy controls for a single tract. The classification error was further reduced when multiple tracts were combined, becoming zero with six tracts.

\section{Manuscripts in Progress:}

Caprihan, A., Pearlson, G.D., and Calhoun V.D. (under review). "Application of Principal Component Analysis to Distinguish Patients with Schizophrenia from Healthy Controls Based on Fractional Anisotropy Measurements"

\section{Presentations and Posters:}

Caprihan, A., Pearlson, G.D., and Calhoun V.D. (accepted) “Application of Discriminant Principal Component Analysis to Distinguish Schizophrenic subjects from Normal Controls Based on Fractional Anisotropy Measurements.” International Society for Magnetic Resonance in Medicine (ISMRM), Toronto, 2008. 
Project 8: Auditory Sensory Gating using MEG
Investigator: Vincent Clark (MRN), Robert Thoma, (UNM)
Period of Report: 4/1/07- 3/31/08

Abstract:

Schizophrenia is a debilitating mental illness with a strong neurological basis. Lifetime incidence estimates suggest that schizophrenia can affect as much as two percent of the U.S. population. It is theorized that many of the functional deficits associated with schizophrenia are related to an inability, at a very early stage of processing, to inhibit irrelevant sensory information from taking up scarce neural resources. A failure of gating may result in the "downstream", higher-order cognitive processes being overwhelmed with irrelevant stimulus information, and thus overall functionality is reduced. Gating is reliably impaired in schizophrenia and has been referred to as the most reliable neuroimaging finding in schizophrenia neurophysiological research. The most common means of measuring this phenomenon is via a protocol typically referred to as P50 auditory sensory gating, in which the 50 and 100 millisecond responses in the auditory regions of the brain are attenuated as a repeated auditory stimulus becomes familiar to the listener. This effect is commonly studied using a "paired-click" protocol in which pairs of clicks, 500 milliseconds apart, are transmitted to a subject in an EEG event related potential (ERP) study. The present study employs multiple neuroimaging modalities (MEG, EEG, sMRI, and DTI) in an effort to identify and characterize the neural network underlying sensory gating. Deficiencies in this gating network are then related to multiple neuropsychological measures and symptomology scales.

Preliminary analyses of MEG data suggest that assessment of auditory sensory gating is consistent with previous results and the current literature. For example, data analysis of MEG is complete for groups of two schizophrenia subjects and four normal control subjects. Consistent with the prevailing literature, there is a trend toward left-hemisphere gating impairment in schizophrenia (control mean left hem gating ratio $=0.26$, schizophrenia mean $=.57 ; \mathrm{p}=.085$ ), and there is a difference between right hemisphere means in the appropriate direction, although it does not currently approach significance with the current $\mathrm{N}$ (control mean right hem gating ratio $=0.35$, schizophrenia mean $=.81 ; \mathrm{p}=.254$ ). Interestingly, the latencies of the $\mathrm{P} 50 \mathrm{~m}$ peaks are later for the schizophrenia grouping the left hemisphere (for $\mathrm{S} 1, \mathrm{t}=\mathrm{-}$ $24.95, \mathrm{p}=.002$; for $\mathrm{S} 2, \mathrm{t}=-8.68, \mathrm{p}=.013$ ), a finding consistent with the left hemisphere impairment hypothesis of schizophrenia, but one not yet reported for the sensory gating paradigm in the literature. We expect to further investigate this finding as our research groups approach the total $\mathrm{N}$ and as our analyses mature. Most importantly, this project has provided needed resources to adapt the sensory gating protocol for MIND instrumentation and software capability. This has been successfully done, and we have resolved many issues and problems via this grant mechanism which has resulted in successful grant proposals.

\section{Objectives and Findings:}

A total of nine subject's complete data (EEG/MEG/MRI/Neuropsychological testing) has been collected (of a total of 20 proposed) and one subject's data collection is currently underway. We ran into several unexpected problems during the course of this project that have each resulted in minor delays. The first was that MIND was not able to provide the GRA support promised for this project and the PI (Thoma) was not able to recruit subjects at the planned rate without support. Resources have been diverted from the PIs other projects to overcome this issue. Further, recruitment of the schizophrenia group was compromised by a miscommunication with the UNM Mental Health Center researchers who had originally promised to help with recruitment. We will necessarily need to recruit subjects from the community. This process has begun, but has resulted in considerable delay. We expect we will finalize data collection in approximately six months time. 
At present, a diagnosis of schizophrenia is based on clinical interviews and description of symptomology. There is no neurophysiological test able to differentiate schizophrenics from non-schizophrenics.

Deficient sensory gating has been referred to as the most reliable neuroimaging finding in schizophrenia neurophysiological research, and is thus a natural choice for use as a diagnostic tool. One of the goals of our research is to develop a sufficiently robust characterization of gating in schizophrenia that an individual subject's gating response can be informative to a clinician in making a schizophrenia diagnosis. The first step of that work, a detailed mapping the nature of gating deficits in schizophrenia, is currently underway. The next step (to begin when data on a sufficient number of subjects has been gathered) aims at the development of a user-friendly algorithm to be applied to electrophysiological data that can reliably distinguish schizophrenic from non-schizophrenic individuals. Spatial and temporal information from multiple imaging modalities (MEG, EEG, sMRI, DTI) will be integrated into the initial diagnostic model, with the ultimate goal of achieving unimodal (i.e., more cost-effective) diagnostic validity.

\section{Manuscripts in Progress:}

Hippocampal lesions abolish P50 sensory gating in humans

\section{Presentations and Posters:}

M.A. Monnig; A. Caprihan; D. Ruhl; P. Lysne; C. Gasparovic; V. Clark; R.A. Yeo; R.J. Thoma, "Diffusion Tensor Imaging Reveals Callosal White Matter Abnormality In Alcohol Dependence And Recovery," 2008 Joint RSA/ISBRA Scientific Conference, June 28 - July 2, 2008, Grand Hyatt Washington DC 
Project 9: Investigation of the Neural Mechanisms Underlying Facial Emotion Recognition
Investigator: Dr. Vincent Clark (MIND), Dr. Robert Thoma (UNM)
Period of Report: 4/1/07-3/31/08

Abstract:

In 1872 Darwin first proposed that social signaling of information concerning psychological state in complex organisms was an evolved trait. In primates and humans, much emotional signaling occurs via facial expression. Indeed, deficient abilities to display and decode emotional signals are considered to be central symptoms of several psychological disorders. Despite the significance of emotion in human psychology, even such simple questions as the number and definition of independent emotional states remain unresolved. The growing availability of neuroimaging techniques in the past decade has led to the identification of specific neural regions involved in both face and emotion processing. Although several neural models have been proposed to explain facial expression processing, results do not conclusively support any single model. Currently, the state of the neuroimaging field in facial emotion perception is centered on the location of major sources of activation during the processing of facial expression, with the hope of being able to link these regions into neural circuits. Specific regions of interest proposed by current models to respond differentially to facial expression are the striate cortex, fusiform gyrus, superior temporal sulcus, and anterior cingulate cortex. Differential activation of a magnetic dipole in the righthemisphere fusiform gyrus when viewing happy versus disgusted versus neutral faces was found in an MEG study by Lewis, et al (2003). Preliminary findings of a larger, follow-up study by Lewis and colleagues have also identified a dipole in the anterior cingulate when viewing happy, sad, angry, disgusted, and neutral faces. The proposed work seeks to extend these findings by, 1) expanding the stimulus set to include all six basic emotions, 2) including a stimulus category of visually complex, nonface images in order to control for the effect of visual complexity, 3) investigating gender differences at the level of neural responding to facial expression, and 4) searching for correlations between neuroimaging findings and several measures of anxiety and depression symptoms.

\section{Objectives and Findings:}

To date, eighteen human subjects have participated in this experiment, including the collection of both structural MRI and MEG data as well as clinical diagnostic forms related to symptoms of anxiety and depression. MRI data has been processed into three-dimensional digital models using the Freesurfer software, and MEG data has been averaged using CTF and MNE software. Cortical projections of the MEG results have been created using MNE, and time courses have been extracted for the right and left hemisphere calcarine fissure and fusiform gyrus areas. Data is currently examined and cleaned prior to input into statistical analysis. An example of the time course data for the right fusiform gyrus is included below. 


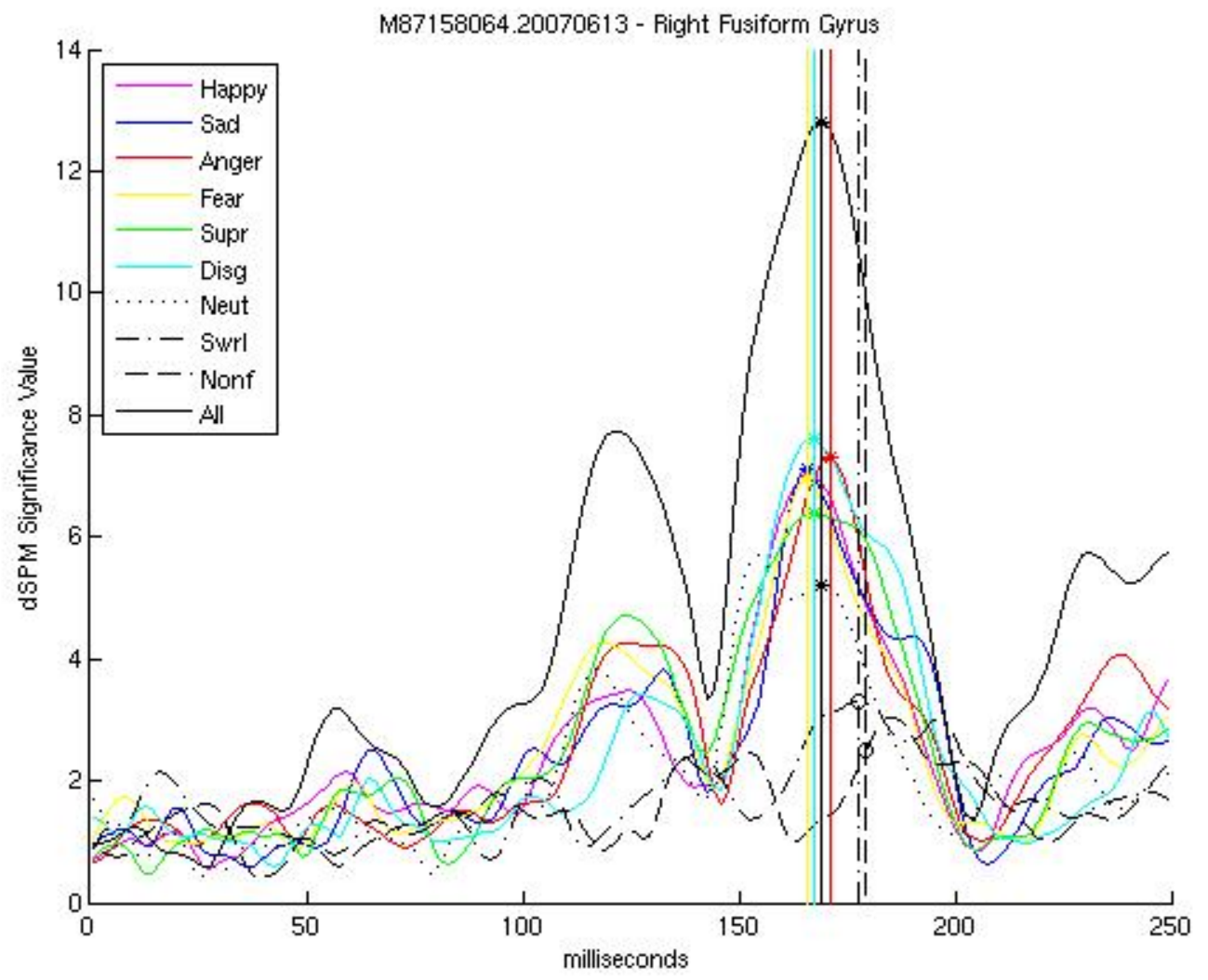

\section{Manuscripts in Progress:}

"Investigation of the Neural Mechanisms Underlying Facial Emotion Recognition, A Thesis Proposal." Per A. Lysne, The University of New Mexico, Department of Psychology \& The MIND Institute, June 18, 2007.

Lysne, P.A., Thoma, R.J., Clark, V., Weisend, M. (in preparation). Neural Dynamics of Face Emotion Processing. In preparation for Journal of Cognitive Neuroscience.

\section{Presentations and Posters:}

"A Magnetoencepahalographic Investigation of the Neural Mechanisms Underlying Facial Emotion Recognition” MIND Institute, September 2007.

\section{Submitted Grant Proposal:}

"Dynamic Modeling of Fusiform Activation in Facial Expression Recognition." Per A. Lysne. National Science Foundation Graduate Research Fellowship Program. 


\author{
Project 10: Effect of Cigarettes on fMRI Studies of Schizophrenia Patients \\ Investigator: Vincent P. Clark, Ph.D. \\ Period of Report: 4/1/07-3/31/08
}

\begin{abstract}
:
Functional magnetic resonance imaging ( $\mathrm{fMRI}$ ) may provide information useful for the diagnosis and treatment of schizophrenia. To date, over 120 peer reviewed articles have been published that examined differences in fMRI responses in schizophrenia. These studies have provided important insights into the causes of and potential treatments for the illness (Kircher \& Thienel, 2005). However, none of these previous studies have examined the contribution of smoking and nicotine use to the results obtained. As much as $85 \%$ of patients with schizophrenia smoke and many are very heavy smokers (Goff et al., 2005). Because cigarette smoking may affect vascular responses, and the fMRI signal results in large part from vascular responses, it is critical to differentiate the physiological changes which are primary to schizophrenia from those that result from cigarette smoking. The focus of this research is to examine the effects of cigarette smoking on fMRI studies of schizophrenia patients.
\end{abstract}

\title{
Objectives and Findings:
}

Objectives for this period were met as data analysis, manuscript preparation, and submission have been completed. Submission to the journal "Schizophrenia Research" is awaiting review. Data analysis, findings and conclusions are as follows. METHODS: Data from actively smoking schizophrenia patients were compared with patient non-smokers who were matched for age, years since diagnosis, medication, and gender, and also compared with data from matched healthy non-smoking controls. Subject mean age was 33 and the smoking group had a mean smoking history of 17 pack years (packs per day x number of years). Blood oxygen level dependent response magnitude and spatial extent were measured within the auditory and sensorimotor cortices, cerebellum, and supplementary motor area. RESULTS: Behavioral responses were not different between groups. The magnitude and spatial extent of activation were largest for control non-smokers, intermediate for schizophrenia smokers, and lowest for schizophrenia nonsmokers, although these differences were not significant when corrected for multiple comparisons. CONCLUSIONS: These results suggest that typical levels of smoking in schizophrenia patients results in a weak but non-significant alteration of the BOLD response in simple sensorimotor tasks when confounds of patient demographics and task difficulty are carefully controlled.

\section{Manuscripts in Progress:}

Leonard Leyba, Andrew R. Mayer, Randy L. Gollub, Nancy C. Andreasen, \& Vincent P. Clark (under review). Smoking status as a potential confound in the BOLD response of patients with schizophrenia. Schizophrenia Research.

\section{Presentations and Posters:}

Dissertation Defense, University of New Mexico Biomedical Sciences, Department of Neuroscience, October 23, 2007.

MIND Research Network Presentation. October 19, 2007. 


\section{Project 11: Multimodal Assessment of Lateralized Hippocampal Function in Schizophrenia Investigator: Faith Hanlon, Ph.D. Period of Report: 4/1/07-3/31/08}

\section{Abstract:}

The hippocampus has long been known to be important for memory, with the right hippocampus particularly implicated in visuo-spatial memory and the left in verbal/narrative or episodic memory. Despite this hypothesized lateralized functional difference, there has not been a single task that has been shown to be able to dissociate the activity of the right and the left hippocampus using neuroimaging. This ability to dissociate the two hippocampi would be an invaluable tool for assessing lateralized hippocampal function in a variety of clinical populations, such as schizophrenia. The transverse patterning (TP) task is a strong candidate for this purpose, as it has been shown in human and nonhuman animal studies to theoretically and empirically depend on the hippocampus. In TP, subjects choose between stimuli presented in pairs, with the correct choice being a function of the specific pairing: $\mathrm{A}+\mathrm{B}-, \mathrm{B}+\mathrm{C}-, \mathrm{C}+\mathrm{A}-$, where the subject is to choose the stimulus marked "+". In this project, the TP task was used to assess right and left hippocampal function in humans by varying its dependence on verbal material, with the goal of dissociating the two hippocampi. Control subjects performed the verbal (pronounceable nonsense words) and nonverbal (abstract pictures) versions of TP while collecting magnetoencephalographic (MEG) and functional magnetic resonance imaging (fMRI) data to verify and validate the tasks' lateralized dependence on the hippocampus. Data were also recorded from schizophrenia patients to determine whether they exhibited a lateralized hippocampal deficit. The neuroimaging data were collected at the Mental Illness and Neuroscience Discovery Institute. The MEG analysis was conducted using the standardized Low-Resolution Brain Electromagnetic Tomography (sLORETA) method in Curry software and the fMRI analysis was conducted using AFNI software. Initial MEG results show that eight of nine controls activated the right hippocampus during the nonverbal version of TP, and eight of nine controls activated the left hippocampus during the verbal version of TP. Importantly, patients showed less lateralized hippocampal activation, and their mean level of behavioral performance was poorer than controls' on both versions of TP. fMRI results averaged across three controls and four patients also showed activation of the right hippocampus during nonverbal TP and left hippocampus during verbal TP compared to a fixation baseline. Importantly, patients had no decrement in performance on a verbal and nonverbal version of the non-hippocampal-dependent elemental task. These preliminary data demonstrate the capacity to discriminate right and left hippocampal function and suggest a bilateral hippocampal deficit in schizophrenia.

\section{Objectives and Findings:}

1. To dissociate right and left hippocampal function using multimodal neuroimaging (magnetoencephalography (MEG) and functional magnetic resonance imaging (fMRI)) and a verbal and nonverbal version of the hippocampal-dependent task transverse patterning (TP) in control subjects. In addition, comparisons between two neuroimaging methods, MEG and fMRI, will be done to evaluate the similarity of their hippocampal localizations. The investigator predicted that both MEG and fMRI would show lateralized hippocampal activation during performance of both versions of TP in controls; the right hippocampus would be activated during the nonverbal version of TP and the left hippocampus would be activated during the verbal version of TP.

This specific aim was supported by the data collected. MEG data showed eight of nine control subjects activating right hippocampus during nonverbal TP (latency $\mathrm{M}=472 \mathrm{~ms}$ ) and eight of nine controls activating left hippocampus during verbal TP (latency $\mathrm{M}=500 \mathrm{~ms}$ ). fMRI results averaged across three controls and four patients also showed activation of the right hippocampus during nonverbal TP and left hippocampus during verbal TP compared to a fixation baseline $(p=.001)$. 
Therefore, both MEG and fMRI data showed lateralized hippocampal activation during performance of both versions of TP as predicted.

2. To characterize the lateralization of hippocampal dysfunction in patients with schizophrenia. Schizophrenia patients will undergo the same TP tasks completed by the control subjects. Behavioral measures, as well as MEG and fMRI neuroimaging, will help establish hippocampal task dependence and the timing and localization of abnormal hippocampal function in schizophrenia patients. The investigator predicted that schizophrenia patients would show a hippocampal-dependent behavioral impairment that was not lateralized. They would be impaired on both the verbal and nonverbal versions of the TP task compared to controls. In addition, schizophrenia patients would show bilateral hippocampal activation for both versions of TP using fMRI and MEG.

As predicted schizophrenia patients required more trials to reach criterion for nonverbal $(p=.034)$ and verbal $(p=.010)$ TP than controls. Patients also had a lower percent correct while performing the nonverbal $(p=.024)$ and verbal $(p=.022) \mathrm{TP}$ in the MEG compared to controls. No differences were found for either measure or version of the non-hippocampal-dependent elemental task. MEG data showed eight of nine control subjects activating right hippocampus during nonverbal TP (latency $\mathrm{M}=472 \mathrm{~ms}$ ) and eight of nine controls activating left hippocampus during verbal TP (latency $\mathrm{M}=500$ $\mathrm{ms}$ ). Five of seven schizophrenia patients activated left hippocampus during nonverbal TP (latency $\mathrm{M}=538 \mathrm{~ms})$, instead of the right found in controls $(\chi 2(2)=4.96, \mathrm{p}=.042)$. However, for the verbal version this difference in laterality was not seen, with five of seven patients exhibiting left hippocampal activation (latency $M=544 \mathrm{~ms}$ ). fMRI results averaged across three controls and four patients showed activation of the right hippocampus during nonverbal TP and left hippocampus during verbal TP compared to a fixation baseline $(\mathrm{p}=.001)$.

Therefore, schizophrenia patients exhibited a behavioral impairment on both versions of TP, indicating a bilateral hippocampal deficit. Controls activated their right hippocampus during nonverbal TP and their left hippocampus during verbal TP. This finding supports the ability of TP to dissociate the two hippocampi by varying the tasks' dependence on verbal material. Unlike controls, schizophrenia patients activated left hippocampus for both verbal and nonverbal TP (replicating nonverbal results in Hanlon et al., 2005). This may result from the left hippocampus trying to compensate for a right hippocampal deficit, using a verbal strategy, although inefficiently, and the right hippocampus unable to do the same for the left. Overall, successful noninvasive lateral differentiation of hippocampal function discovered abnormal lateralization in schizophrenia that may reflect a compensatory effort or a reduction in lateral functional specialization. These results are consistent with literature showing unusual brain asymmetries in schizophrenia (Crow, 1997; Petty, 1999; Yeo et al., 1999).

The investigator originally proposed to have 10 controls and 10 schizophrenia patients recruited and run on both MEG and fMRI by the end of the project period. Unfortunately, this goal was not reached due to a reduction in the percentage of time allotted to the PI to work on the project. However, MEG and fMRI data was successfully collected on a smaller dataset than proposed, in which the results are described above, and used as preliminary data in two submitted NIH grants, an R21 grant entitled "Multimodal Assessment of Lateralized Hippocampal Function" (1 R21 MH078966-01; PI: Hanlon) and a COBRE grant entitled "Neural Mechanisms of Schizophrenia: Use of Multiple Neuroimaging Tools to Examine Dysfunctions in Neural Integration" (1 P20 RR021938-01A1; PI: Lauriello), with Hanlon as a PI of a subproject entitled "Fronto-temporal Coherence: A Test of the Disconnection Hypothesis in Schizophrenia". 


\title{
Manuscripts in Progress:
}

Hanlon, F.M., Jones, A.P., Bantz, R.M., Lundy, S.L., Thoma, R.J., Weisend, M.P., Mayer, A.R., Bustillo, J.R., Miller, G.A., \& Tesche, C.D. (In preparation). "Assessment of Lateralized Hippocampal Function in Schizophrenia"

\section{Presentations and Posters:}

“Assessment Of Lateralized Hippocampal Function In Schizophrenia” at the International Congress On Schizophrenia Research (ICOSR), 03/28/2007.

\section{Project 12: Structural Mechanisms of Convergent and Divergent Cognitive Processes in Humans. \\ Investigator: Rex E. Jung, Ph.D. \\ Period of Report: 4/1/07-3/31/08}

\begin{abstract}
:
In this project, we outline a program of research designed to articulate the neuroscience of creativity. By carefully assessing uniquely human characteristics associated with the creative process - including cognitive measures of convergent and divergent thought processes, behavioral measures of creative achievement and artistic talent, and measures of spontaneous versus more effortful brain processes - we endeavor to link discrete brain regions and modes of neuronal operation with a construct that, although central to human experience, has remained largely intractable to the inquiries of science over the last century. To our knowledge, no individual or group is undertaking a systematic approach to understanding the nature of creativity using comprehensive neuroscience techniques. Our hope is that this project will stimulate external funding support for research regarding the important interrelationships between brain functioning and this important behavioral construct. We propose to combine structural and functional neuroimaging paradigms to better understand the interplay between brain structure, cognitive function, and behavioral manifestations associated with creativity.
\end{abstract}

\section{Objectives and Findings:}

Our objectives were to collect sufficient pilot data to submit a competitive grant application to the National Science Foundation, John Templeton Foundation, or other entities in the area of the neuroscience of creativity. We aim to determine macro- and micro-structural and biochemical correlates of creativity using structural Magnetic Resonance Imaging (sMRI), Diffusion Tensor Imaging (DTI), and proton Magnetic Resonance Spectroscopic Imaging $\left({ }^{1} \mathrm{H}-\mathrm{MRSI}\right)$. Two contrasts were experimentally evaluated - the first (Brain-Cognition) assessing structural correlates of divergent cognitive tasks, the second (Brain-Behavior) assessing structural differences between "ordinary" creative individuals (undergraduates) compared to "uncommonly" creative individuals (e.g., visual artists).

During the past funding period, we collected data on 75 experimental subjects, analyzed spectroscopic, diffusion tensor imaging, and morphometric data, submitted a grant to the John Templeton foundation, and won a grant for $\$ 600,770$ to study "The Neuroscience of Creativity" based on our preliminary data. We now have a three-year program of study (7/17/07-7/1710), entirely funded by external means, to explore the neuroscience of creativity based on a DOE initial investment. Moreover, we have established 
the first results reported in the area linking morphometric, DTI, and spectroscopic measures to the behavioral construct of creativity (see Presentations and Posters below). Dr. Jung is emerging as a leader in the field and has been invited to present at a National Science Foundation sponsored workshop entitled "Studying Design Creativity: Design Science, Computer Science, Cognitive Science and Neuroscience Approaches" in March 2008. His laboratory has had accepted seven abstracts to date in 2007/2008 at conferences including the Society for Neuroscience, Cognitive Neuroscience Society, Human Brain Mapping, and International Neuropsychological Society. Papers are in various stages of preparation, with one In Press, and several in preparation or in submission. This area of research has expanded the MIND portfolio into the realm of "positive psychology" defined as "what the brain does well", and including such investment areas as intelligence and giftedness, creativity, neuroplasticity, and accelerated learning.

\section{Manuscripts in Progress:}

Haier RJ \& Jung RE (In Press). Brain imaging studies of intelligence and creativity: What is the picture for education? Roeper Review.

Jung RE (In Press) Towards A Neuroscience of Creativity. Conference Proceedings of Studying Design Creativity: Design Science, Computer Science, Cognitive Science and Neuroscience Approaches.

Jung RE, Kalbfleisch ML, Haier RJ, Bockholt HJ, Segall J, \& Yeo RA. (Under Submission). Neuroanatomy of Creativity.

\section{Presentations and Posters:}

Jung RE, Kalbfleisch ML, England RL, Bockholt HJ, Barrow R, Segall JM, Paar MH, Smith SM, Chavez R, \& Haier RJ. Neuroanatomy of Creativity. Presented at the 37th annual meeting of the Society for Neuroscience, San Diego, CA, 3 - 7 November 2007.

Verney SP, Jung RE, \& Garcia C. Sustained Attention on the Work Sample Test is Associated with Cognitive Ability. Presented at the $36^{\text {th }}$ Annual International Neuropsychological Society Meeting, Waikoloa, HI, 6 - 9 February, 2008.

Bennett J, Verney SP, \& Jung RE. Assessing Creativity and Intelligence in an Ethnically Diverse Sample. Presented at the $36^{\text {th }}$ Annual International Neuropsychological Society Meeting, Waikoloa, HI, $6-9$ February, 2008.

Chavez R, Caprihan A, England R, Smith S, Segall S, Barrow R, \& Jung RE. Corpus Callosum Contributions to Creativity: A Diffusion Tensor Imaging Study. Presented at the $15^{\text {th }}$ Annual Meeting of the Cognitive Neuroscience Society, San Francisco, CA, 12-15 April, 2008.

Jung RE, Barrow R, Chavez R, Smith S, \& Gasparovich C. Biochemical Correlates of Creativity. Presented at the $15^{\text {th }}$ Annual Meeting of the Cognitive Neuroscience Society, San Francisco, CA, 12-15 April, 2008.

Caprihan A, Barrow R, Chavez R, Bockholt H.J. \& Jung RE. Correlation of White Matter integrity Measured by DTI with Intelligence, Personality, and Creativity in Healthy Subjects. Presented at the $14^{\text {th }}$ Annual Meeting of the Organization for Human Brain Mapping, Melbourne, Australia, 15 - 19 June, 2008.

Jung RE, Bockholt HJ, Segall J, Caprihan A, Chavez R, Smith S, \& Kalbfleisch ML. Creative Achievement and Cortical Thickness in a Large Healthy Cohort. Presented at the $14^{\text {th }}$ Annual Meeting of the Organization for Human Brain Mapping, Melbourne, Australia, 15 - 19 June, 2008. 


\section{Project 13: Structural, Diffusivity, and Biochemical Brain Mechanisms of Broad Cognitive Decline in Schizophrenia \\ Investigator: Rex E. Jung, Ph.D. \\ Period of Report: 4/1/07-3/31/08}

Abstract:

We propose to use high field ( 3 Tesla) multimodal imaging to investigate whether general cognitive functioning in schizophrenia is related to circuit level white matter, metabolic, and volumetric changes in subcortical gray- and white-matter regions suggestive of fronto-subcortical disconnection. Simultaneous utilization of Diffusion Tensor Imaging (DTI), proton magnetic resonance spectroscopy $\left({ }^{1} \mathrm{H}-\mathrm{MRS}\right)$, and structural Magnetic Resonance Imaging (sMRI) will allow us to relate white matter abnormalities to both cortical and subcortical metabolic and morphological changes that, in turn, may underlie ongoing neurocognitive decline in schizophrenia. As schizophrenia is postulated to be a disease that involves the integration of information among distributed brain circuits, we propose to use DTI, ${ }^{1} \mathrm{H}-\mathrm{MRSI}$ and sMRI to investigate: 1) whether fronto-subcortical network abnormalities are present in schizophrenia, 2) whether cognitive functioning in schizophrenia is related to circuit level dysfunction, and 3) whether regions beyond the identified brain circuits contribute significantly to broad cognitive functioning characteristic of schizophrenia. Diffusion Tensor Imaging, a measure of axonal/myelin integrity based on water diffusion within cellular compartments, will be measured within the superior longitudinal fasciculus, cingulum bundle, and anterior limb of the internal capsule (ALIC); N-acetylaspartate (NAA), a marker of metabolic dysfunction or neuronal loss/integrity, will be measured within the anterior cingulate gyrus, frontal white matter, ALIC, caudate nucleus, and thalamus; structural MRI, yielding a measure of cortical thickness, will be measured within the anterior cingulate gyrus and Brodmann areas 9/46 \& 10. A hierarchical approach will be explored, in which cognitive functioning is predicted by cortical thinning, white matter microstructure changes, and metabolic changes within discreet fiber tracts in SP. We will also test the hypothesis that ongoing neurocognitive impairment in schizophrenia is related to chronic disconnection of fronto-subcortical circuits, reflected in morphological contraction of discrete frontal lobe regions critical to higher cognitive functioning. Finally, we will explore whether specific genes associated with the formation, development, and functioning of myelin are associated with circuit level dysfunction in SP. No research to date has simultaneously explored diffusivity, metabolic, and morphometric abnormalities within these critical brain circuits as they relate to neurocognitive dysfunction in schizophrenia. We see this as a critical line of investigation that will help us understand and potentially intervene in the ongoing cognitive dysfunction that currently limits the ability of treated patients to return to more normal occupational and social functioning.

\section{Objectives and Findings:}

Our objectives were to collect sufficient pilot data to submit a competitive grant application to the National Institutes of Health to support a Center Grant (COBRE) application that was previously scored favorably (below 200), but not in the fundable range. This project was designed to collect data and develop techniques to present in a third and final revision, the results of which will be known in the Spring of 2008. As we had acquired a new 3 Tesla Siemens scanner, it was important to show data

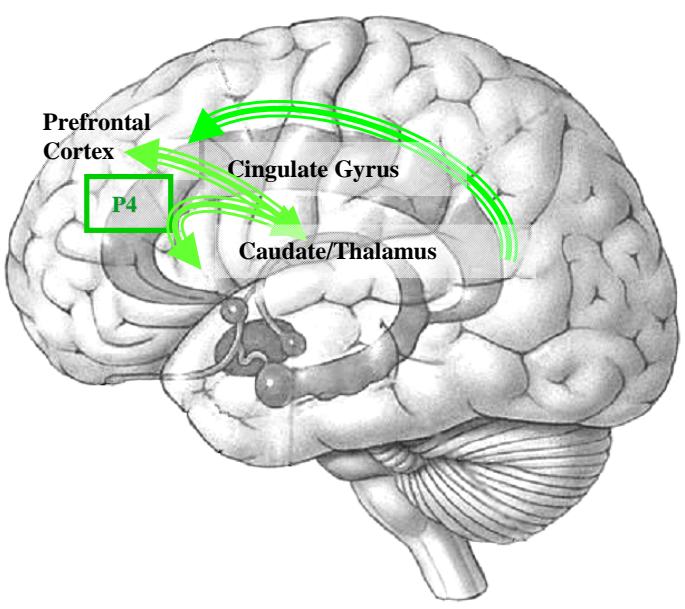


acquired from that platform, which we acquired in five schizophrenic and five control subjects.

During the past funding period, we collected data on 5 experimental subjects and 5 controls, analyzed data from these subjects, incorporated the results into the new COBRE submission, and continued to publish in the area of schizophrenia (see Publications section). Should this NIH project be funded, it would represent a substantial return on the 8,818.50 investment made by DOE to collect pilot data. Moreover, the investment made has yielded several publications/presentations in high impact scientific journals and meetings to date.

\section{Manuscripts in Progress:}

Bustillo JR, Rowland LM, Jung RE, Brooks WM, Qualls C, Hammond R, Hart B, \& Lauriello J. (In Press) Proton magnetic resonance spectroscopy during the first year of antipsychotic treatment in schizophrenia. Neuropsychopharmacology

Bustillo JR, Rowland LM, Mullins PG, Jung RE, Chen H, Qualls C, Hammond R, Brooks WM, \& Lauriello J. (Under Review). 1H-MRS at 4 Tesla in Minimally Treated Early Schizophrenia.

White T, Magnotta V, Bockholt HJ, Williams S, Gollub RL, Mueller B, Ho BC, Schulz SC, Andreasen NC, Calhoun VC, Jung RE, \& Lim KO. (In Preparation). Global White Matter Abnormalities in Schizophrenia: A Multicenter Diffusion Tensor Imaging Study.

\section{Presentations and Posters:}

Ruhl DA, Thoma RJ, Bockholt HJ, Monnig M, Jung RE, Lauriello J, \& Bustillo J. Cerebellar Cortical Volume and Executive Functioning in Schizophrenia. Presented at the $36^{\text {th }}$ Annual International Neuropsychological Society Meeting, Waikoloa, HI, 6 - 9 February, 2008.

Bockholt HJ, Williams S, Scully M, Magnotta V, Gollub R Lauriello J, Lim K, White T, Jung RE, Schulz C, Andreasen N, \& Calhoun V. The MIND Clinical Imaging Consortium as an application for novel comprehensive quality assurance procedures in a multi-site heterogeneous clinical research study. Presented at the $14^{\text {th }}$ Annual Meeting of the Organization for Human Brain Mapping, Melbourne, Australia, 15 - 19 June, 2008. 
Project 14: Application of Novel Neuroimaging Techniques to the Study of Dementia
Investigator: Andrew Mayer, Ph.D.
Period of Report: $4 / 1 / 07-3 / 31 / 08$

Abstract:

Dementia is a medical condition resulting in cognitive and functional decline including memory loss and inability to provide self-care. As life expectancy and the prevalence of elderly individuals continue to increase, the possibility of a world-wide pandemic of cognitive impairment becomes more likely. It is estimated that nearly 81 million people will suffer from this disorder world-wide in 2040. Dementia encompasses a broad spectrum of disorders with different neuropathologies and, therefore, different treatments. However, current diagnostic approaches are frequently insensitive to the neuropathology that separates Alzheimer's dementia (AD) and Vascular dementia (VaD) from healthy aging, and alternative techniques are needed to 1) improve diagnosis, 2) identify in-vivo markers of disease-specific neuropathology, and 3) identify specific domains of functional and cognitive decline, which will ultimately lead to better treatment. Recent advances in MRI, including diffusion tensor imaging (DTI) and the application of blind source separation techniques (independent components analyses) to resting state FMRI data, will increase the clinician's ability to distinguish between $\mathrm{AD}$ and $\mathrm{VaD}$ and to predict different profiles of cognitive impairment and everyday functioning across these two groups based on the putative mechanisms of injury: neurodegeneration of mesial temporal structures in AD contrasted with demyelinating/inflammatory and ischemic injuries in $\mathrm{VaD}$. Critically, objective methodology for selecting the default mode network and for integrating measures of white and gray matter connectivity must also be pursued before this work can be fully implemented with clinical populations.

\section{Objectives and Findings:}

The overall goals of the project were achieved through three separate platforms: 1) a large study of normal controls for determining the methods most appropriate for quantifying the default mode network, 2) the determination of appropriate methodology for combining FMRI resting state and DTI data, and 3) parallel pursuits for studying the same phenomenon in patients with dementia and matched elderly control.

To achieve objective 1, we examined different methodologies for identifying resting state data. Specifically, there has been a growing interest in the neuroimaging community regarding resting state data (i.e., passive mental activity) and the subsequent activation of the so-called default mode network

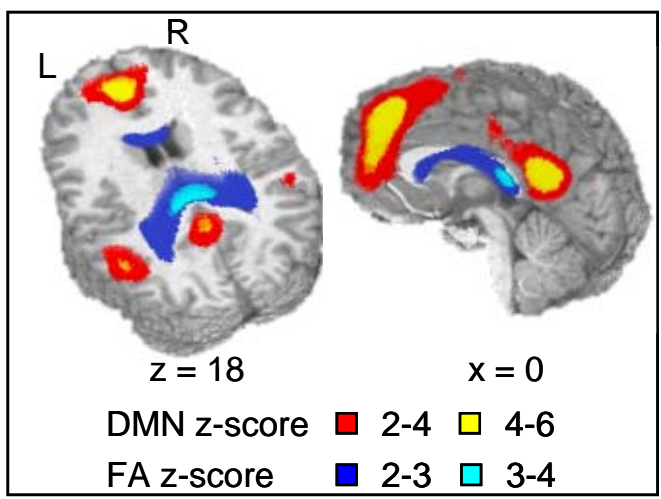

Figure 1: J-ICA of resting state and DTI data
(DMN). Although this network was originally characterized by a pattern of deactivation during active cognitive states, more recent applications of data driven techniques such as independent component analysis (ICA) have permitted the analyses of brain activation during extended periods of truly passive mental activity. However, ICA requires the resultant components to be evaluated for "goodness of fit" via either human raters or more automated techniques. To our knowledge, an investigation on the reliability of either technique in determining the component that best corresponds to default-mode activity has not been performed. Moreover, it is not clear how automated techniques, which are necessarily dependent upon a template mask, are affected by the structures used to compose the mask. Therefore, we investigated both inter-rater (human- 
human) reliability and inter-method reliability (human-machine) for determining DMN activation in 42 healthy controls. Results indicated that near perfect inter-rater reliability was achieved, whereas intermethod reliability was only within the moderate range. However, the latter was significantly improved via a weighted combination of the anterior and posterior cingulate nodes of the DMN. More recently, we examined novel statistical methods for comparing resting state and DTI data (objective 2), which represent measures of gray and white matter connectivity, respectively. Specifically, we used joint-ICA to determine regions that exhibited shared variance across the two neuroimaging techniques. Our results (Franco et al., manuscript in preparation) indicate that there was a high correlation between resting state activity and the magnitude of fractional anisotropy within the corpus callosum (Figure 1). This suggests that passive mental activity is highly related to inter-hemispheric white matter connectivity.

To achieve objective 3, twenty-three subjects ( $\mathrm{VaD}, \mathrm{AD}$ and matched controls) have been assessed with a

Table 1. Subjects enrolled in pilot behavioral study.

\begin{tabular}{llllll}
\hline Group & $\mathrm{n}$ & Age & Educ. (yrs) & MMSE & Global T-Score \\
\hline $\mathrm{NC}$ & 13 & 77 & 15 & 28.2 & 48.3 \\
$\mathrm{VaD}$ & 4 & 85 & 12 & 22.5 & 36.0 \\
$\mathrm{AD}$ & 6 & 75 & 15 & 17.7 & 30.0 \\
\hline
\end{tabular}

MMSE=Mini Mental Status Exam; Global T-score = Mean of demographically Corrected $\mathrm{T}$-scores for the 15 neuropsychological test scores ( $\mathrm{T}=50$ is normal)

\begin{tabular}{llllll}
\hline \multicolumn{5}{c}{ Table 2. Corpus Callosum Fractional } & Anisotropy \\
\hline \multirow{3}{*}{ Control } & Genu (1) & $(2)$ & $(3)$ & $(4)$ & Splenium (5) \\
VaD & 0.56 & 0.49 & 0.50 & 0.59 & 0.68 \\
\hline
\end{tabular}
neuropsychological test battery, and four subjects have completed the neuroimaging protocol. Preliminary results (Table 1) indicate that both the $\mathrm{VaD}$ and $\mathrm{AD}$ groups are more impaired on the neuropsychological test battery than the controls. The default mode network has been identified in these participants as depicted by the methods presented in objective 1. Table 2 displays FA values for several regions in the corpus callosum, indicating lower FA for $\mathrm{VaD}$ patients relative to a control. This preliminary work has been submitted for funding and we are awaiting our score.

\section{Manuscripts in Progress:}

Franco, A.R., Pritchard, A., Calhoun, V.D. \& Mayer, A.R. (submitted). Inter-rater and Inter-method Reliability for Selecting the Default Mode Network during Data-Driven Analyses. Human Brain Mapping.

Franco, A.R., Ling, J. Caprihan, A., Calhoun, V.D. \& Mayer, A.R. (in preparation). Novel methods for assessing gray and white matter connectivity using multi-modal neuroimaging. IEEE. 


\title{
Project 15: A Multimodal Study of Sensory Gating in Schizophrenia Investigator: Andrew Mayer, Ph.D. Period of Report: 4/1/07-3/31/08
}

\begin{abstract}
:
Multiple lines of research have consistently documented sensory processing deficits in patients with schizophrenia (SP). One such deficit is poor sensory gating, in which there is an increased electrophysiological response for the second of two rapidly presented stimuli in SP compared to healthy normal volunteers $(\mathrm{HNV})$. This effect is typically reported in terms of a gating ratio comparing the amplitude of the response for both the first (S1) and second (S2) stimulus (S2/S1*100). Poor sensory gating has been characterized as both a deficit in selective attention and/or in the formation of memory traces, and is a useful bio-marker of the cognitive and subsequent social dysfunction that is typically observed in SP. Electroencephalography (EEG) and magnetoencephalography (MEG) studies of sensory gating have implicated the temporal lobes, including the superior temporal gyrus, as the most likely neuronal generator of the sensory gating deficit. Although other invasive neuroimaging techniques suggest a role for the hippocampus and prefrontal cortex in sensory gating, neither EEG nor MEG studies have revealed hippocampal activation. While this negative result may be reflective of a true lack of hippocampal activation in gating, it may also be secondary to technical limitations with EEG and MEG, such as poor spatial resolution, failure to examine appropriate time epochs, and/or difficulties localizing sources in deep structures under certain conditions. In contrast, functional magnetic resonance imaging (FMRI) is not restricted by any of these limitations, suggesting that it may be an alternative mechanism for studying the entire neuronal network underlying the gating deficit, including mesial temporal and prefrontal sources. The current application proposes to use several neuroimaging modalities, including MEG, EEG and FMRI to identify where and when the sensory gating deficit occurs in schizophrenia. The combination of multi-modal imaging techniques should lead to a more complete understanding of the complex neuronal pathology underlying schizophrenia and its cognitive manifestations. In the current protocol, SP and HNV will be presented with identical clicks, identical tones and non-identical tones while undergoing functional imaging. We are predicting that this will produce a double dissociation of functioning with SP exhibiting higher gating ratios for the identical tones and clicks conditions and lower gating ratios for the non-identical tones condition whereas HNV will exhibit the opposite pattern. The functional data from these conditions will be directly compared and also correlated with behavioral and neuropsychological measures to further quantify the relationship between brain function and the observed clinical pathology which characterizes this basic inhibitory failure. Finally, impaired sensory gating is believed to be an endophenotypical marker for schizophrenia. Therefore, we will also investigate the genetic contribution to our measures of electrophysiological and hemodynamic functioning in both HNV and SP.
\end{abstract}

\section{Objectives and Findings:}

This project was initially conducted as part of the MIND Institute's Center of Biomedical Research Excellence (COBRE) grant. The overall objectives of the project were achieved through three separate platforms: 1) two resubmissions of our COBRE grant, 2) resubmission of a NIH grant examining sensory gating in a population of chronic cocaine abusers, and 3) finalizing data collection in healthy controls to determine the robustness of the paradigm.

The first two goals of this research program involved seeking extramural support for the conductance of gating research in two populations with known deficits in basic inhibitory processes. Specifically, the study of sensory gating was a core project in The MIND Institute's attempt to hierarchically document cognitive dysfunction in SP using multiple neuroimaging modalities. Our first COBRE submission received a priority score of 189 . We resubmitted this COBRE grant in the spring of 2007 and received a 
second priority score of 160 , which was just outside of the fundable range. The third and final submission of the COBRE occurred in November of 2007 and we are awaiting our final priority score. The PI of this project also submitted a grant examining sensory gating deficits in chronic cocaine abusers to determine whether the gating response could be used to predict relapse compared to other clinical measures. This grant was funded in the fall of 2007; we are currently finalizing the protocol in anticipation of data collection beginning in April of 2008.

In addition to these first two aims, we collected extensive pilot data to verify that the gating paradigm, which has typically been used in electrophysiological studies, could be utilized for FMRI. Specifically, a more complete understanding of the neural generators involved in normal sensory gating is critical for elucidating the pathological response that is often observed in clinical populations. Animal data have consistently suggested the involvement of the primary auditory cortex, prefrontal cortex and hippocampus in models of sensory gating. However, electrophysiological methodologies have typically only observed sources within the superior temporal gyrus, which may be a result of limitations with this technique. Therefore, we utilized a variant of the paired click paradigm in which pairs of identical and non-identical tones were presented to examine the effects of distinct, compared to repeated, stimuli. To our knowledge, a FMRI study of sensory gating using the parameters which are commonly employed in electrophysiological research (i.e., 3-5 ms stimulus duration, $500 \mathrm{~ms}$ ISI; 8-10 s ITI) has not been conducted and is an important first step for the proposed translational research. Electrophysiological results from a similar paradigm indicate larger gating effects for the identical (P50 gating ratio $=44 \%$ ) compared to non-identical tones condition (P50 gating ratio $=67 \%)$ in normal controls, with a reversal of effects in patients with schizophrenia. Although the temporal resolution of the hemodynamic response is limited, we were able to directly compare the additive summation of the responses to pairs of identical and non-identical stimuli as a more direct measure of sensory gating. We also compared statistically summed hemodynamic responses to single tone stimuli. We predicted that both identical and nonidentical tones would involve a similar network of structures including the primary auditory cortex, hippocampus, and prefrontal cortex but that the neuronal response would be greater in these regions for the non-identical tones indicative of a reduced gating response. Our results indicated that while the primary auditory cortex and dorsolateral prefrontal cortex were both activated bilaterally for the statistically summed hemodynamic response, no differences emerged between the comparison of nonidentical and identical tones.

\section{Manuscripts in Progress:}

Leyba, L., Mayer, A.R., Gollub, R.L., Andreasen, N.C., Clark, V.P. (submitted). Smoking status as a potential confound in the BOLD response in patients with schizophrenia. Biological Psychiatry. 
Project 16: Attentional Functioning in Normal and Neuropsychiatric Populations

Investigator: Andrew Mayer, Ph.D.

Period of Report: 4/1/07-3/31/08

\section{Abstract:}

Attentional deficits are prevalent amongst many neuropsychiatric disorders including Alzheimer's dementia, Vascular dementia, traumatic brain injury (TBI) and schizophrenia. However, a more thorough understanding of how attention works in normal controls is critical before these paradigms can be successfully employed in different patient populations. The overarching goal of the project is therefore to improve the current understanding of the neuronal mechanisms that underlie selective attentional functioning and spatial orienting in both healthy controls and neuropsychiatric populations. Specifically, humans are constantly bombarded with multiple streams of information in both the auditory and visual that must be ignored or selected for future action. Selective attention permits the integration of this multisensory information when it is congruent and differential processing of this information when it is incongruent. Spatial attention refers to human's ability to increase the processing of information presented

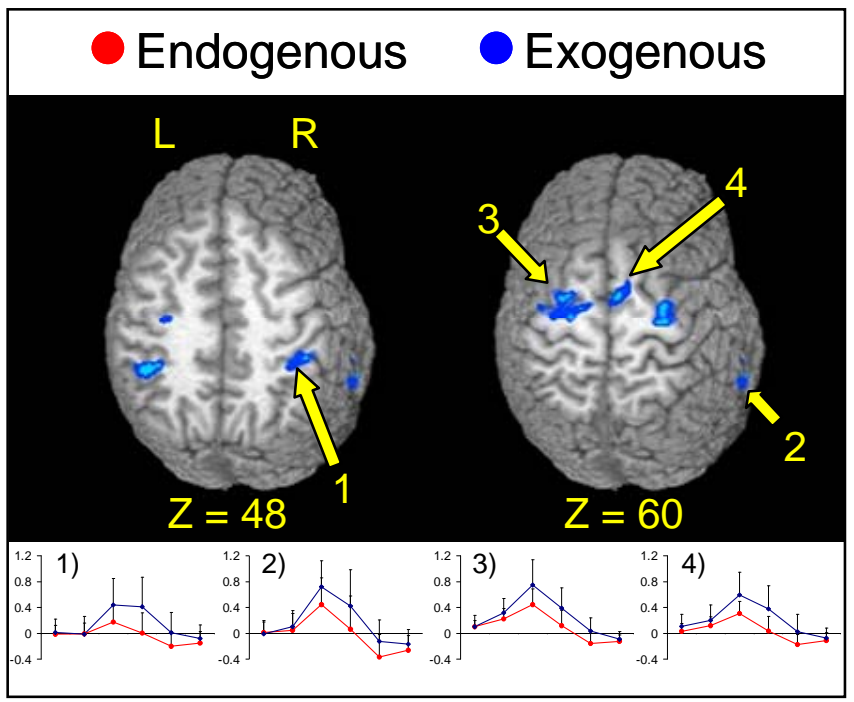

Figure 1: Results from auditory orienting study. at a specific location in space. There are competing demands for the spatial control of attention from stimuli in the outside (exogenous) world, such as flashing signs or loud noises, as well as from internally-driven goals (endogenous) or cognitions, such as a desire to look left or to listen more attentively to conversation that is occurring at the adjacent table. Deficits in attention directly result in cognitive, social and interpersonal impairment, which is detrimental not only to the identified patient, but also to their immediate care-givers and the community as a whole. No where are these attentional deficits more apparent than in patients who have suffered a TBI. The current proposal reviews the PI's progress in the neurological foundations of attention both in healthy normals and patients with TBI. Our work

with patients with schizophrenia and dementia are presented in the separate sections that follow.

\section{Objectives and Findings:}

This project is representative of the programmatic research of the principle investigator over the past several years. The overall goals of the project were achieved through three separate platforms: 1) an increased understanding of the neuronal mechanisms underlying exogenous compared to endogenous auditory attention, 2) an increased understanding of how humans use selective attention to process conflicting auditory and visual information with a high temporal component, and 3) the application of these techniques to the study of mild TBI. 
The endogenous versus exogenous orienting of attention has reliably produced dissociable behavioral and neuronal effects within the visual modality as shown by our group, (Arrington, Carr, Mayer, \& Rao, 2000; Mayer et al., 2004a; Mayer et al., 2004b) and others. Moreover, the orienting of attention to following either endogenous auditory cues (Mayer et al., 2006) or exogenous auditory cues (Mayer et al., 2007) has also been investigated by our group. However, how humans allocate attention based on auditory cues with low (exogenous) or high (endogenous) cue validity has not been directly compared. Our primary result (Figure 1) was of increased activation for exogenous compared to endogenous auditory cues in the 1) inferior parietal lobe, 2) middle temporal gyrus, 3) bilateral frontal eye fields and 4) pre supplementary motor areas (Mayer et al., submitted). Collectively, our current and previous results on the exogenous and endogenous orienting of auditory attention suggest that while a supramodal network may support attentional control in both primary sensory modalities, the way that this information

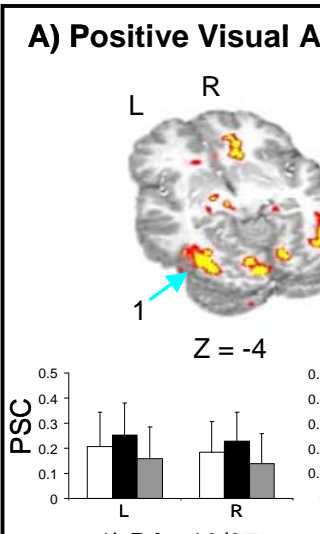

1) BAs $19 / 37$

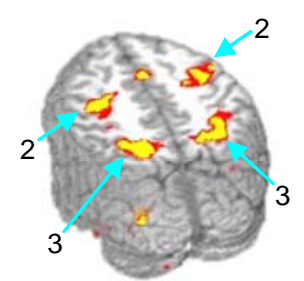

$Z=46$

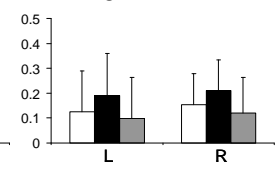

3) BAs $39 / 40$

\section{B) Negative ARMs}
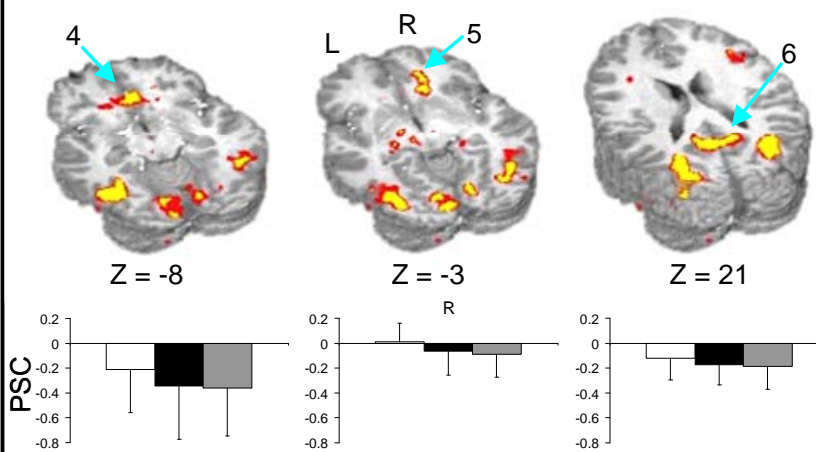

4) BAs $11 / 32$

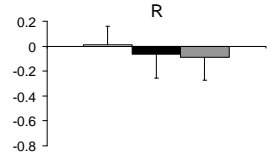

5) Anterior cingulate gyrus

$\square$ MTMD

AVIS

$\square$ AAUD

Figure 2: Results from first selective attention study.

is processed is highly dependent on the modality in which the information originates, the temporal proximity between two attended events, and behavioral expectancies about events. At shorter time intervals, auditory cues may promote a more automatic orienting response than visual cues, resulting in large reorienting effects due to the reflexive allocation of attention resources. At longer SOAs, this may be followed by increased activation for valid trials due to the presence of either an inhibitory bias or reduced reorienting effects. More automatic control mechanisms might be engaged by auditory information because it is processed more rapidly and as such, may serve as a warning system to alert organisms of changes in the environment, followed by a more volitional and thorough analyses of the environment via the visual system. This simple formulation, while requiring more extensive testing and evaluation, may offer a parsimonious explanation for the differential orienting effects observed between the two sensory modalities. We have previously performed FMRI imaging studies on the Stroop task (Mead, Mayer, et al., 2002) and conducted behavioral studies of selective attention in a spatial task for both normal (Mayer \& Kosson, 2004) and neuropsychiatric populations (Mayer et al.,

2006). In 2007, we extended this work by completing two experiments (one submitted a manuscript) investigating selective attention and multisensory integration using a novel paradigm. The first experiment required participants to ignore multisensory stimuli that occurred at different frequencies (.5, 1 or $2 \mathrm{~Hz}$ ) and the results are presented below. The second experiment utilized a numeric Stroop in which conflicting numeric information was presented across the two modalities and served as a foundation for the TBI work presented below. Our results (Mayer et al., submitted) from the first study revealed several potential neural mechanisms for the dominance of auditory signals over visual stimuli when selectively attending to temporal information in a multimodal context. When stimulus rate was equated, attention related modulations (ARMs) were observed within the attend-visual, but not the attend-auditory condition, within widely distributed dorsal and ventral frontal, parietal, and occipital streams (Figure 2). 
The functional results, however, depended in part on the rate of stimulus information; this pointed to the power of parametric manipulations of stimulus properties in uncovering underlying brain mechanisms. For example, evidence for the obligatory recruitment of auditory cortex was found under conditions in which ignored auditory information was particularly detrimental to task performance, as when attending to a relatively slow-pace visual metronome (e.g., ignore-auditory condition at $.5 \mathrm{~Hz}$ ) where there was a larger window of time to for conflicting information to intrude upon attention processes. In contrast, in primary and secondary cortical areas, ARMs were present for both the attend-visual and attend-auditory condition, but only at $2 \mathrm{~Hz}$. Here, the pattern of results suggested conflicting signals in the ignored modality were easier to suppress when selectively attending to rapidly paced information. Finally, both the current results and other research support the recruitment of a more extensive cortical network during rhythmic tapping to visual cues with or without auditory distractors. Collectively, these results indicate that the processing of auditory information during tasks with a high temporal component is more efficient (i.e., requires coordination of a smaller network), less effortful (i.e., decreased variability and presence of ARMs) and sometimes obligatory (i.e., automatic recruitment of auditory cortex during both attend and ignore conditions).

Finally, the PI of these studies also started a TBI research program. This endeavor resulted in seven grant applications to various funding agencies (NIH and DOD) in 2007, one of which received pilot funding. The primary objective of the funded grant is to prospectively collect neuropsychological and imaging data (FMRI, MRS, DTI) on patients with mild TBI both during the semi-acute (within 3 weeks) and more chronic phases (3-5 months post injury) of injury. To date, 6 adults with mild TBI have completed the protocol for the semi-acute phase of injury (less than 3 weeks post-injury) and one subject has completed both phases of data collection. Following experimental completion, we will have a neurochemical assay (MRS) and measures of white matter integrity (FA values) for the majority of the corpus callosum, the internal capsule and the prefrontal white matter. Likewise, we will have hemodynamic measures of gray matter functioning and a neurochemical assay for

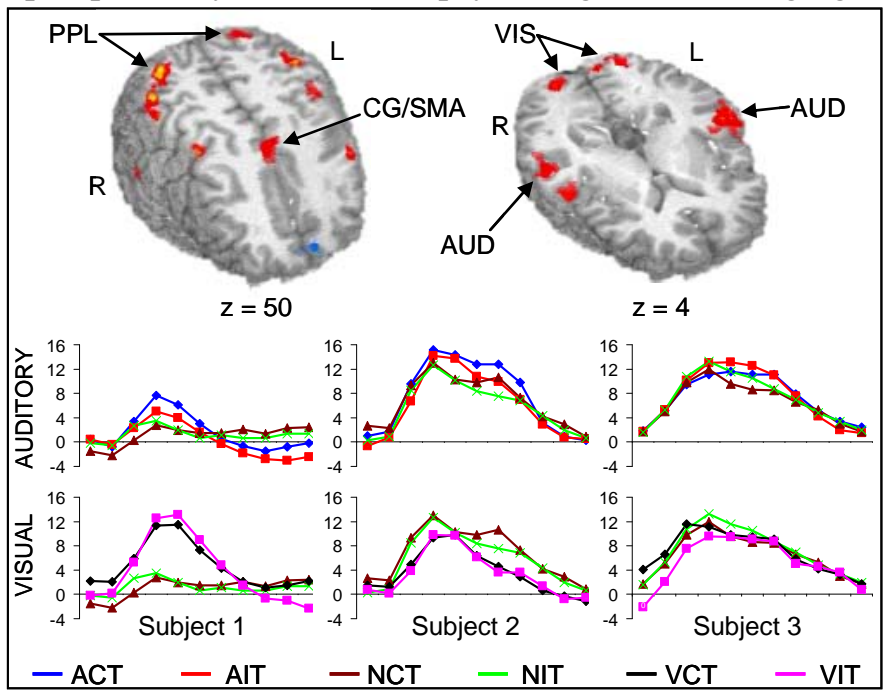

Figure 3: Results from $3 \mathrm{TBI}$ patients. the anterior cingulate area. The two FMRI tasks for this study are based on auditory orienting and selective attention paradigms presented above, both of which have also been shown to be deficient in mild TBI. There were six conditions in the selective attention task. Participants were asked to attend and identify numbers occurring in either the auditory or visual modality while ignoring a congruent or incongruent crossmodal distractor that occurred at two separate frequencies. In the two control conditions, participants passively listened to the congruent or incongruent stimuli presented in both modalities without responding. Mean results (averaged $\mathrm{Z}$ score of 1.5 across subjects) indicate that the unisensory cortical areas, posterior parietal lobes (BAs 7/40), dorsolateral prefrontal cortex and anterior cingulate gyrus/ pre-supplementary motor area (BA 32) were all activated by the incongruent conditions. The derived IRFs for the congruent (ACT and VCT), incongruent (AIT and VIT) and control conditions (NCT and NIT) are presented for the auditory and visual unisensory cortical areas for 3 TBI subjects during the semi-acute phase of injury (Figure 3 ). In general, all subjects showed small attentional effects (i.e., greater amplitude of response for congruent or incongruent trials compared to control condition) for the auditory modality. However, only subject 1 demonstrated the expected attentional modulation (VCT $>$ NCT and VIT $>$ NIT) within the visual 
modality, suggesting that subjects 2 and 3 were unable to properly allocate cognitive resources in a challenging multisensory environment.

\title{
Manuscripts in Progress:
}

Mayer, A.R., Franco, A. \& Harrington, D.L. (submitted). Neuronal Modulation of Auditory Attention by Informative and Uninformative Spatial Cues. Human Brain Mapping.

Mayer, A.R., Harrington, D.L., Franco, A., \& Canive, J. (submitted). The Effects of Stimulus Modality and Frequency of Stimulus Presentation on Cross-Modal Distraction. Cerebral Cortex.

Project 17: Neurochemistry of Brain Activity in Response to Pain

Investigator: Paul Mullins, Ph.D.

Period of Report: 4/1/07-3/31/08

\begin{abstract}
:
Pain is a major adaptive response to injury or harm and has a definite positive role in the short term, but when pain persists far beyond the period of initial injury it becomes debilitating and detrimental to a persons health and well-being. It is estimated that about 50 million people in the United States suffer from persistent, serious pain (Marx, J. 2004). Response to pain involves both peripheral and central mechanisms with several systems, neurotransmitters and receptors playing a role in pain transmission and response (Besson, J. M. 1999). How acute pain in response to an injury or disease transitions to a chronic pain syndrome, and the underlying neurobiology of pain are still poorly understood. This project proposed to extend previous findings using functional Magnetic Resonance Spectroscopy (fMRS) in pain by linking changes in neural activation to changes in concentration of the neurotransmitter glutamate and one of it's intermediates, glutamine, in response to pain. The ability to measure such activation and neurotransmitter increases will provide a novel surrogate marker for categorizing pain syndromes and assessing patient pain status not to mention determining neurotransmitter interactions and monitoring potential therapeutic and analgesic treatments.
\end{abstract}

\section{Objectives and Findings:}

The project design called for subjects to undergo functional magnetic resonance imaging (fMRI) and proton magnetic resonance spectroscopy before, during and after an acute painful episode, hypothesizing that painful states will cause an increase from baseline levels in neural activity, and hence glutamate transmission and concentration in specific regions of the brain, in particular, the anterior cingulate and the thalamus. The proposed research plan and actual accomplishments follow.

Phase 1: Preliminary feasibility and protocol testing, HRRC/IRB approval and setup were in place in the spring 2007. Installation of the Siemens 3T scanner required for this study was completed by 03/27/2007. Implementation of the particular MRI and MRS protocols to be used in this study were completed by mid June, and three subjects run through the paradigm in a small pilot study to ensure the protocols all worked. 
Phase 2: Recruitment of subjects and acquisition of data. Subject recruitment was to be run in parallel with subject recruitment from Dr Mullin's ongoing fibromyalgia studies conducted in collaboration with Dr. Bruce Smith (UNM). Data acquisition was to take place from the end of March until September, or until all subjects had been collected. As the spectroscopy protocols were not fully in place until mid June, data acquisition was delayed slightly. In addition, Dr. Mullins left the MIND Institute at the end of July to take up a position at Bangor University in North Wales. As a result the study was suspended; however, the preliminary data was used in an RO3 grant application submitted to NIH in late July. This application secured an award and the project is being performed by MIND with Dr. Mullins participating from his new post. Preliminary data relating to the spectroscopic technique also led to a recent submission to "Magnetic Resonance in Medicine" and was accepted for presentation at the International Society for Magnetic Resonance in Medicine's conference in 2008.

\title{
Project 18: Application of Neuroimaging Techniques to Improve Methods of Matching Children with Dyslexia to Reading Intervention Investigator: Juliana Paré-Blagoev,, Ph.D. Period of Report: 4/1/07-3/31/08
}

\begin{abstract}
:
Between 5-15\% of Americans have dyslexia, a difficulty learning to read which is biologically based and unexplained by IQ or socio-environmental factors (Lyon et al., 2003). For those with dyslexia, trying to read is akin to deciphering an enigmatic code and repercussions go far beyond difficulties in classroom academics. There are social problems associated with consistent school failure and/or excessive academic struggles. In addition, as students graduate into the workforce, employers rarely provide an environment which is as supportive as that of the classroom; so failures can multiply. Dyslexia is a tough problem to try to solve, in part because learning to read is a complex process and things can go wrong at multiple points or combinations of points. Further, across people the brain may compensate in different ways even if the originating neural deficits are the same. This heterogeneity presents a real challenge when trying to choose appropriate interventions for specific children.
\end{abstract}

\section{Objectives and Findings:}

We proposed to characterize the neural correlates of phonological awareness skills which are critical to learning to read to a more precise degree than has currently been attempted. We planned to use paradigms based on phonological awareness assessments and (in the future) on phonological awareness interventions to investigate whether neuroimaging can be used to develop diagnostic methods for matching dyslexic children to the reading interventions that can help them the most. In most cases, researchers, clinicians and the teachers on the front lines do not have a basis for matching a child with dyslexia to the best possible treatment. Thus far, no neuroimaging studies have been conducted to address this question and no behavioral studies have provided guidelines for doing this matching.

During the past funding period, we initiated the HRRC process on this study. In anticipation of the transition to the 3T scanner we postponed data collection on this project and developed a complementary study to this one. Like the dyslexia study, the pediatric Autism study described below was based on an approach which harnesses the reliability of widely used behavioral, clinical assessment tools with the 
intent of developing diagnostic paradigms that link imaging approaches and clinical behavioral tools. Prior to the availability of the 3T scanner, the PI on this project transitioned to a new position outside of the MIND Institute. Funding on this project was halted at that point.

The complementary autism study developed is described below: fMRI and MEG studies have been used in the past to study Mirror Neuron System performance in children and adults with autism. The proposed imaging research utilizes both of these neuroimaging tools to address unanswered questions about the MNS functioning in children with ASD. Unlike past studies, aspects of our paradigms are tightly related to clinically used assessment and/or intervention tools. We anticipate that this will hasten the translation of these imaging results to clinical applications.

Autism is a Pervasive Developmental Disorder (PDD) characterized by repetitive motions, and by qualitative impairments in reciprocal social interaction and communication (APA, 1994). It is thought to affect 1 in 166 children (Fombonne, 2003), and appears to be 4 times as prevalent in boys than in girls (Osterling and Dawson, 2004). Mirror neurons and their connection to autism are of increasing interest to the scientific community and potentially to clinical efforts as well. Mirror neurons are neurons that fire both when an animal or human observes an action performed by another, as well as when the animal or human performs the same act. They were first identified in single-neuron studies performed on macaque monkeys (Gallese, et al., 1996). While those original studies identified mirror neurons in the premotor cortex (area F5) of the monkey, subsequent studies have shown that they are also present in the monkey inferior parietal lobule (Rizzolatti, et al., 2001). In humans, homologous areas have been found within the pars opercularis of the inferior frontal gyrus (Broca's area) (Iacobini, et al., 1999). From a functional standpoint, studies have suggested that mirror neurons can connect the visual representation of an action with the motor representation, an association that could lead to imitative behaviors (Rizzolatti, et al., 2001). In addition to imitation, mirror neurons are also believed to function in action understanding, social interaction, empathy, and language. Because many of these functions are known to be impaired in autism, the Mirror Neuron System is of interest to autism researchers as a possible neural cause of the social impairment seen in ASD.

Previous studies implementing various techniques and approaches have shown strong support for the hypothesis that mirror neuron dysfunction is connected to autism. For example, studies have found imitative deficits in autism involving simple body movements and symbolic actions (Rogers and Pennington, 1991), as well in imitating facial expressions (Loveland, et al., 1994). These older studies identified specific deficits in autism, while newer studies have implicated the mirror neuron system (MNS) in this dysfunction.

The primary objective of this work is to pursue research investigating Mirror Neuron System (MNS) involvement during passive observation and imitation of facial expressions, and during observation and execution of body movements in individuals with Autism Spectrum Disorder. Functional magnetic resonance imaging (fMRI) and magnetoencephalography (MEG) during task performance will be used in combination with a series of neuropsychological tests that will be administered outside of the scanning environment. 


\author{
Project 19: \\ Constraint Therapy For Children With Hemiplegic Cerebral Palsy \\ Cortical Visual Impairment in Children with Cerebral Palsy \\ Investigator: John Phillips, M.D. \\ Period of Report: 4/1/07-3/31/08
}

\begin{abstract}
:
These studies are part of our overall effort to develop new neuroimaging methods appropriate for children with brain disorders, particularly targeted toward understanding and improving neurologic treatments.

For the constraint project, the study investigates the feasibility of using fMRI as a physiologic marker of brain plasticity before and after an intensive constraint-induced movement therapy (CIMT) program in children 6-14 yrs of age with cerebral palsy (CP). Six children with spastic hemiplegic CP and three agematched normal controls will be recruited. Children with $\mathrm{CP}$ will receive CIMT twice daily (four hrs total) for two weeks. fMRI scans using a hand squeezing stimulus will be completed pre- and postintervention. Clinical measures of hand and arm function, self care and parent goals will also be used. Statistical analyses to demonstrate change will include repeated measures ANOVAs and post hoc analyses. By using a novel approach to performing fMRI in children with cerebral palsy that we have recently developed, this will be the first study investigating the neurologic mechanism of clinical improvement in CIMT. With pilot data obtained from this study, we hope to proceed to larger studies powered to answer questions about efficacy and optimal use of constraint induced movement therapy in children with cerebral palsy.
\end{abstract}

In preparation for both studies, we conducted a study in 16 children with hemiplegic or diplegic CP to determine 1) how best to reduce head movement during ankle dorsiflexion fMRI and 2) whether subjects can be prescreened before imaging to determine whether excessive movement will occur. Children were 6 to 19 years old, mean 10.75 years, and gross motor function classification system level (GMFCS) I = 4, GMFCS II $=10$, GMFCS III $=2$. Screening was performed by asking subjects to lie supine and dorsiflex their most involved ankle as required for the fMRI experiment for 5 minutes, and during this time the investigator observed for head movement. All subjects were then scanned using 3 different frequencies of ankle dorsiflexion in a block design. In order to monitor the amount of force exerted by the children, a specialized force plate and transducer was developed.

\title{
Objectives and Findings:
}

Using the screening method adopted for this study of children, we were able to correctly predict which children would successfully complete the MR experiment without excessive head movement in 15 of 16 subjects (i.e., only one out of 5 failed datasets were not predicted beforehand). Unsuccessful children were GMFCS level I $=1$, GMFCS level II $=3$, and GMFCS level III $=1$. Secondly, we evaluated the effect of ankle speed on head movement. Three imaging experiments were performed at $0.5,0.33$ and $0.25 \mathrm{~Hz}$ of ankle dorsiflexion. Head movement was followed throughout the entire experiment in 6 directions as indicated below in a sample fMRI run. By summing total movement, we found that most subjects had the least head movement at $0.5 \mathrm{~Hz}$, although 3 subjects had lest movement at the $0.33 \mathrm{~Hz}$. None did best at $0.25 \mathrm{~Hz}$.

There are three significant findings of this study: I) The first aspect of this study demonstrates that 2/3rds of our children with CP could successfully complete the MR studies. In addition, we were successful in predicting who would provide good quality datasets in the large majority of children (15/16).

Furthermore, one of the two GMFCS level III subjects successfully completed the scan sequences. II) The second part of this study shows that for all our subjects either ankle dorsiflexion at 0.5 or 0.33 hertz (but 
not 0.25 hertz) minimizes excessive head movement. The current proposal thus uses these two frequencies for the fMRI scans. III) The final significance of this study is that it shows the feasibility of performing force analysis during scanning. This is important because it provides an objective measurement of performance that can be used to ensure consistent effort in follow up scans. Trials producing excessive force can be discarded, thus ensuring that changes in cortical activation (either intensity or location) are not simply due to differences in performance. Thus, for any neuroimaging involving children, force analysis should be considered.

We applied this information to a clinical study of constraint therapy in children with hemiplegic cerebral palsy. Our target goal of 9 subjects was met including control children as well as those that received intensive constraint therapy for two weeks. Working with the Department of Occupational Therapy, the children underwent detailed motor assessments which will be compared with imaging data (this includes fMRI, DTI, structural imaging and force analysis). Data analysis is currently ongoing.

Manuscripts in Progress:

“Ankle Dorsiflexion fMRI in Children With Cerebral Palsy"

Project 20: High Field fMRI

Investigator: Kamil Ugurbil, Ph.D.

Co-Investigators: Dr. Pierre-Francoise Van de Moortele (UMN), Dr. Gregor Adriany (UMN), Dr. Vincent Clark (MIND)

Period of Report: 4/1/07-3/31/08

\section{Abstract:}

The general goal of this project is the development of improved functional imaging methodologies using potential gains that can be harnessed from ultrahigh magnetic fields (7T or above), and examination of mechanisms underlying BOLD and perfusion based functional imaging towards ultimately establishing high resolution and high specificity methods for imaging of brain activity. The activities undertaken to achieve this specific aim will be centered around (a) the development of improved ultra high magnetic field hardware (7 and 9.4 Tesla instruments, RF coils, multichannel receivers etc) for anatomical, functional and diffusion tensor imaging in the human brain, (b) examination of mechanisms underlying imaging signals for mapping brain function, (c) optimization of functional imaging techniques at ultrahigh magnetic fields for the ultimate goal of routine acquisition of high resolution and high accuracy fMRI maps in the whole brain at 7 and 9.4 Tesla (d) development of proton spectroscopy methods at ultra high magnetic fields for investigating functional correlates of tissue bioenergetics, and (e) the use of this methodology to support studies in normal and diseased human brain.

\section{Objectives and Findings:}

The objectives of this project were:

1. To develop new strategies for high resolution and high specificity BOLD functional mapping.

2. To develop multislice spin-echo encoding scheme for volume coverage that minimizes SAR and time of volume coverage.

3. To improve implementations of 3 Dimensional (3D) EPI sequences for GE BOLD MRI. 
4. To develop improved transmit/receive arrays for achieving high acceleration factors with parallel imaging.

In all of these areas significant progress has been made. Three new sequences are beig worked on for high resolution functional imaging. These include novel encoding strategies, as well as improved implementation of already established concepts.

Under Objective 1, we employed a newly designed slab-selective T2 prepared sequence, which consists of a 90 degree pulse followed by a 180 degree refocusing pulse, and then a -90 degree pulse to flip the magnetization back along the z-axis. Delays surrounding the 180 degree pulse encode T2 weighting. This preparation is followed by rapid gradient echo acquisition of multiple slices. It is critical that the acquisitions are as rapid as possible, so that gradient echo (GE) effects do not come significantly into the functional imaging signals and that many slices can be acquired before the magnetization preparation is altered significantly (which occurs in the time scale of T1). This process can be repeated $\mathrm{N}$ times for a multiple number of slabs. Parallel imaging techniques, with high reduction factors, and RF coils based on array technology to achieve this becomes indispensable for this goal. In addition to parallel imaging gains, this multi-slice Hahn spin echo (HSE) sequence provides an intrinsic reduction in SAR over conventional HSE acquisitions, in which the overall reduction depends on the number of slices achievable after the magnetization preparation. The ratio between the power deposition in a conventional multi-slice HSE and this sequence is: $5 \mathrm{~N} /(\mathrm{N}+6)$, for the same total acquisition time In our first experiment, we investigated the effect of using a longer slab readout duration on normal subjects at 7 T. Experiments were be done using 12, 14, 16, and 18 axial slices through the visual cortex, corresponding to readouts of $282,329,376$, and $423 \mathrm{msec}$ for all of the slices (i.e. $23.5 \mathrm{msec} / \mathrm{slice}$. A matrix size of $128 \times 64$ (FOV: $20 \times 12 \mathrm{~cm} 2$ ), single shot acquisition, and a $2 \mathrm{~mm}$ slice thickness will be used. The TE/TR will be $55 \mathrm{msec} / 2 \mathrm{~s}$. In addition to this, the inflow effect and the corresponding GE contrast was be measured with the longer readout times and thicker slabs. A visual paradigm was be used, consisting of 5 blocks of $30 \mathrm{~s}$ on / $30 \mathrm{~s}$ off, corresponding to 5 minutes per run, using a flashing checkerboard pattern. The relative activation, with and without the T2 preparation for each of the readouts was be quantified and spatially compared, assessing the overall GE weighting to the multi-slice SE sequence. In addition, the thick slab acquisition $(120 \mathrm{~mm})$ was be compared with the activation from the different readouts, to again quantify any inflow effects. The results of this experiment will be used to plan subsequent experiments in terms of pushing the acquisition limits for general applications of this sequence.

As a part of Objective 1, we explored more efficient implementations of 3D GE EPI sequences using parallel imaging techniques to speed up the acquisition time, and in addition we employed physiological corrections to reduce some of the effects of physiological motion. It has been established that for high field fMRI studies, and more specifically for high spatial resolution applications, that GE BOLD images are not generally as good as HSE BOLD images. Despite this apparent problem, success has been achieved with GE BOLD studies investigating lower resolution applications. This is consistent with our results showing that the point spread of the GE BOLD response in the gray matter is better than $2 \mathrm{~mm}$ at 7 T. Furthermore, at low fields GE BOLD remains the attractive option for fMRI given that the spatial specificity and sensitivity of HSE BOLD is undesirable at low fields. The majority of GE BOLD fMRI studies use 2D multi-slice approaches. However, many fMRI applications desire to have contiguous data. With the use of 3D approaches, contiguous whole brain volumes can be obtained with benefits in the signal to noise ratio over 2D studies. However, 3D approaches for fMRI applications can be limited because of the time it takes to image the volume at the desired resolutions and the subsequent physiological noise that is introduced during this time, specifically in human applications.

Advancing Objective 3, subjects were scanned at $7 \mathrm{~T}$. The 3D EPI sequence consisted of a standard 2D EPI sequence, where the third dimension of the excited slab was spatially (phase) encoded. The 3D slab was: $20 \times 20 \times 7.5$, matrix $64 \times 64 \times 24$, yielding an isotropic resolution of approximately $3 \mathrm{~mm}$. To avoid 
folded signal contributions from outside the FOV, the actual thickness of the slab was reduced to $85 \%$. Based on our current evaluation of 16 channel array, using the 32 channel coil, a 2D reduction factor of 16 was attained at $7 \mathrm{~T}$, significantly reducing the acquisition time. An echo time of $25 \mathrm{msec}$, and the corresponding Ernst angle of $15^{\circ}$ was used. Baseline fMRI time series were acquired. It has been demonstrated that are significant non-MR sources of noise present in brain images. In addition, it has been shown that noise levels in 3D fMRI experiments may be significantly increased by instabilities in the transverse steady-state magnetization when the TR is on the order of the T2. To further reduce the fluctuations in the 3D EPI time series, RF spoiling were used. For the 2D acquisitions, all the same parameters were used, while exciting each slice separately, and using a 1D reduction factor of 4 to reduce the acquisition time. The raw baseline fMRI data is being processed to reduce respiration induced Bo fluctuations. In addition, the raw data is being processed with a retrospective technique we described previously (physio-fix) to remove fluctuations associated with cardiac pulsation and respiration. The corrected images are undergoing analysis for residual image to image signal fluctuations, by using a power spectrum analysis and quantifying the signal fluctuations in the time series. The $2 \mathrm{D}$ and $3 \mathrm{D}$ images will be compared to assess any improvements in SNR in the 3D data.

The previously described experiments were supported by two sets of RF coil, each with 32 channels:

32 Channel Flexible Geometry Transcieve Coil: The primary coil that we currently rely on is a novel a 16 channel array coil which is flexible and can be adjusted to different human head size. With 2 short elements, the coil allows for easy visibility and ability to present visual input. With this coil, we have experimentally determined that 12 or 16 fold 2D maximum aliasing (hence acceleration) can be tolerated in SENSE acquisition, with excellent and good g-factor performance, respectively. This coil will be used as the basis of a design for a 32 channel array. In this 16 channel coil, at present each element (except for elements over the eyes) is sufficiently long along $\mathrm{z}$ (magnetic field direction) to cover the brain and cerebellum. These long elements will be split in two in the 32 channel coil. We know from the short elements in this coil, that short elements can be made to work in this transmission line design and that they have improved SNR. We expect that linearly arranged two elements of transmission line design will be decoupled using decoupling capacitors.

Lattice Coil: Parallel imaging performance and SNR is expected to increase with the number of coils; a limit on the SNR increase is imposed, however, if increasing number of coils necessitates a progressively decreasing coil size because with small coils, coil resistance starts becoming a dominant source of noise. Similarly, difficulties in decoupling the different array elements with increasing number of coils impose a practical limitation on improvements both in SNR and parallel imaging performance. These limits may be relaxed by concentric coil arrangements. Along these lines, we will pursue a combination of concentric transmission line elements for head imaging to demonstrate that the transmission line elements can be used both in parallel and perpendicular orientation to the magnetic field and to investigate whether an array consisting of a combination of both type of elements improves SNR and parallel imaging performance. Since at such high fields, transmit arrays with a large number of independent channels are of considerable interest for RF transmission, we plan an outer 16 channel transceiver array that allows for independent phase, amplitude and pulse shape control for each array element. This 16 element transceiver array will then be combined with a second 16 channel array of short transmission lines used for reception only, yielding an array with 16 transmit and 32 receive channels. This coil will have distributed elements both radially (in the xy-plane perpendicular to z) and along $\mathrm{z}$. It can be used for 2D acceleration in a 3D acquisition with any choice of the orientations for the phase encoding and readout gradients. Similar improvements can be expected for oblique slices. 


\author{
Project 21: Improved Methodology and hardware for C13 MRS studies \\ Investigator: Kamil Ugurbil, Ph.D. \\ Co-Investigators: Dr. Pierre-Francoise Van de Moortele (UMN), Dr. Gregor Adriany (UMN), Dr. \\ Vincent Clark (MIND) \\ Period of Report: 4/1/07-3/31/08
}

\begin{abstract}
:
Glutamate is the main excitatory neurotransmitter in the brain. Glutamatergic dysfunction has been implicated in a number of neuropsychiatric disorders including schizophrenia (SZ), Alzheimer's disease and depression. Progress in gaining a better understanding of glutamatergic dysfunction in humans has been hampered by the lack of non-invasive methods to measure glutamatergic neurotransmission. Magnetic resonance spectroscopy allows non-invasive measurement of metabolite concentrations and metabolic fluxes in the brain non invasively and in vivo. Carbon-13 NMR spectroscopy (13C MRS) is a unique tool to measure metabolic fluxes non-invasively in the brain. The long-term goal of the proposed research is to develop non-invasive methods based on C13 MRS for the measurement of glutamatergic neurotransmitter turnover rates and to utilize this in animals and humans for studying normal brain bioenergetics, coupling between neurotransmission rates and bioenergetics, perturbations on these processes by pathophysiology and understanding the impact of treatment. At the present, C13 spectroscopy is the only technique capable of measuring glutamatergic neurotransmission rates, a key parameter in evaluating the glutamate hypothesis in mental disease.
\end{abstract}

\title{
Objectives and Findings:
}

The objectives of this project were:

1. Develop novel metabolic modeling approaches for in vivo measurement of metabolic fluxes with $13 \mathrm{C}$ isotopic labeling.

2. Examine and validate these approaches in animal models.

3. Development of $13 \mathrm{C}$ data collection schemes and hardware (RF coils based on transmit/receive arrays) for the whole brain studies in humans.

These three aims in conjunction with the development of new approaches for rapid, high sensitivity 13C isotopic labeling and rapid acquisition of $13 \mathrm{C}$ volumetric imaging to monitor $13 \mathrm{C}$ isotopic labeling kinetics in vivo are essential steps in developing C13 spectroscopy for use in clinical studies. In 2007, we made significant progress for the metabolic modeling. Monte Carlo simulations were used to test the veracity of the existing models as well as the proposed new models. Improved accuracy was predicted using two C13 substrates simultaneously, namely acetate and glucose. Experiments were already conducted in animal models verifying the results. These are at the present being written up. Objective 1 we focused on simulations using the two-compartment models. Nearly all metabolic modeling studies with two-compartment models have been performed using [1-13C]glucose or [1,613C2] glucose as a substrate. The measured turnover curves used for the fitting typically correspond to the total $13 \mathrm{C}$ enrichment at each carbon position (positional 13C enrichment) such as the $\mathrm{C} 4, \mathrm{C} 3, \mathrm{C} 2$ positions of glutamate and glutamine. The accuracy of these models can be evaluated using Monte-Carlo Simulations. Therefore, we focused on simulations using the two-compartment models to fit positional $13 \mathrm{C}$ enrichment curves obtained during [1-13C]glucose or [1,6-13C2] glucose infusion, varying parameters such as the number of experimental data points per turnover curve, the number of fitted turnover curves, or the noise level. In addition, the sensitivity of turnover curves to changes in the value of specific metabolic fluxes can be examined. We took steps to determine the reliability of each fitted metabolic flux in the model depending on simulated experimental conditions. Finally, examined the efficacy of using 13C labeled acetate rather than glucose, to determine if it allow improved kinetic modeling of glutamatergic turnover. This was be tested using Monte-Carlo simulations as well. 
Our preliminary data showed that using glucose as a substrate does not permit precise determination of VNT because most 13C label goes through the big neuronal glutamate pool first, followed by labeling of the smaller pool of glutamine. This result is $13 \mathrm{C}$ labeling time courses that are relatively insensitive to the value of VNT. Labeling the smaller pool of glial glutamine before labeling the bigger pool of neuronal glutamate (eg using [2-13C] acetate) lead to 13C labeling time courses that are more sensitive to the value of VNT. In this project we performed extensive Monte-Carlo simulations and sensitivity analysis to confirm the reliability of VNT (and other fluxes) when modeling 13C time courses obtained during [2$13 \mathrm{C}]$ acetate infusion, depending on experimental parameters such as the total duration of $13 \mathrm{C}$ acquisition or the signal-to-noise ratio. These simulations guided the choice of the voxel size, duration of experiment and temporal resolution.

Subsequent to modeling studies, we implement to advance Objective 2, involved animal model experiments for evaluation and verification of methodology. We focused on data collected in normal animals and subsequent to inhibition of NMDA receptors using phencyclidine (PCP). As an antagonist of glutamate NMDA receptors, PCP provides a means to directly manipulate glutamatergic neurotransmission in an animal model. PCP treatment, especially chronic PCP treatment, has generated tremendous interest as an animal model of schizophrenia and has been instrumental in developing the glutamatergic hypothesis of schizophrenia. We performed preliminary 13C-acetate infusion experiments in normal animals to determine the optimal rate of acetate infusion, and subsequently in normal and PCP challenged animals to obtain glutamate and glutamine labeling kinetics. Blood acetate concentration and enrichment were measured using GCMS. Brain 13C spectra recorded using 13C-edited LASER to measure the brain acetate signal and to verify that time courses of glutamate and glutamine do not depend on the acetate infusion rate above the threshold necessary to avoid washout of acetate from the brain. These simulations and preliminary experiments were used to determine optimal experimental conditions for subsequent studies.

The goal of this Objective 3 was to develop 13C data acquisition schemes and an improved RF coil development. The initial experiments were performed at $7 \mathrm{~T}$. Subsequently, the portability and suitability of these methods examined at $3 \mathrm{~T}$. over the period of performance, we continued to work on development of an array coil for $13 \mathrm{C}$ and $1 \mathrm{H}$ for human brain. Our group has been active since 2003 in developing array coils for 7T. The strategy we have employed was transmit/receive ("transcieve") arrays based on stripline (TEM) technology. In addition to having multiple channel receive capability, this approach has the benefit that the transmit B1 can be modulated in phase and amplitude individually for each array element, allowing for "B1 shimming", and also transmit SENSE. The utility of B1 shimming for high fields where the RF homogeneity is an issue has already been demonstrated in our previous work. Subsequent to our initial publication, we have been working on several different designs which are at different stages of maturity. We continue to refine design of a 13C/1H RF coil based on these existing models. Two designs were examined: an $1 \mathrm{H}$ coil that can be further increased in the number of elements it has by dividing the elements along $\mathrm{z}$ into two, thus improving SNR. In this case, the elements were tuned to $13 \mathrm{C}$. An outer separate concentric and larger coil was built for $1 \mathrm{H}$ for decoupling and $1 \mathrm{H}$ imaging of anatomy. We have already accomplished such concentric rings at $1 \mathrm{H}$ frequency. The two different frequencies in the $13 \mathrm{C} / 1 \mathrm{H}$ coil made this an easier task. 

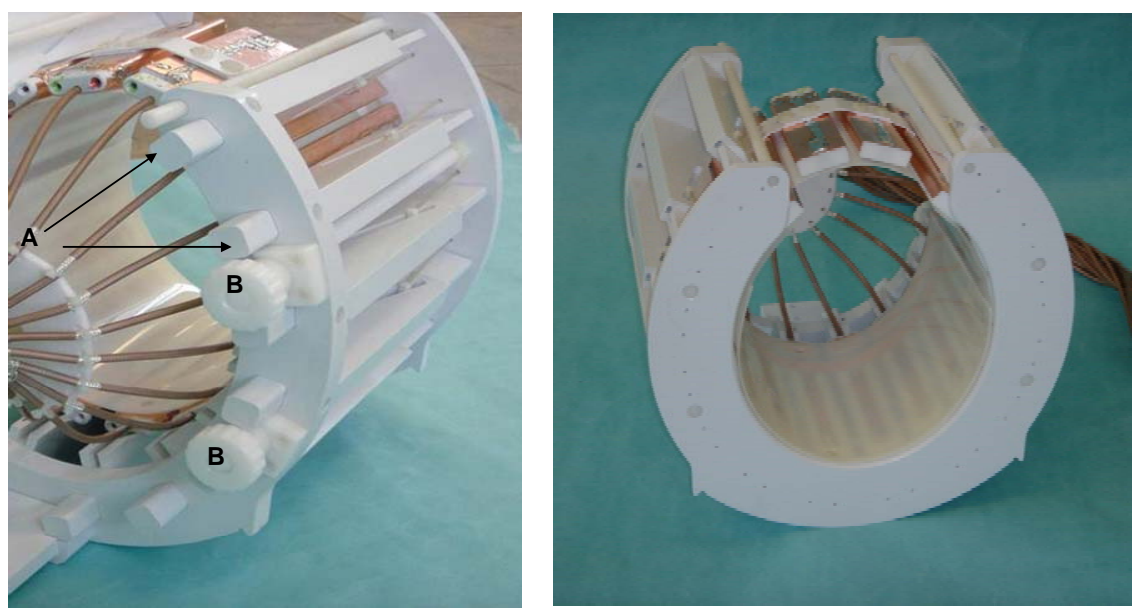

Transceiver array coil with 2 short elements $(10 \mathrm{~cm}), 14$ longer elements $(14 \mathrm{~cm})$; Min Coil size: $17 \mathrm{~cm} \times 21 \mathrm{~cm}$, Max Coil size: $21 \mathrm{~cm} \times 25 \mathrm{~cm}$

A second approach was based on an inner array of overlapping loops for 13C, and an outer larger coil of transmission line elements for $1 \mathrm{H}$.

New Technologies: As described above

\section{Manuscripts in Progress:}

Iltis I, Marjanska M, Du F, Koski DM, Zhu XH, Ugurbil K, Chen W and Henry PG. ${ }^{1}$ H MRS in the Rat Brain Under Pentobarbital Anesthesia: Accurate Quantification of In Vivo Spectra in the Presence of Propylene Glycol. Magnetic Resonance Med. 2008; In Press

Marjanska M, Henry PG, Uğurbil K, Gruetter R. Editing Through Multiple Bonds: Threonine Detection. Magnetic Resonance Med. 2008;59(2):245-251

Olman C, Van de Moortele P-F, Ugurbil K, Yacoub E: Retinoptic Mapping at 7 Tesla: Surface Reconstruction from Intensity-Corrected Anatomical Data Enables Single-Session Experiments. Magnetic Resonance Med 2008; Submitted

Zhang N, Liu Z, He B, Chen W: Noninvasive Study of Neurovascular Coupling During Graded Neuronal Suppression. JCBFM 2008; 28:280-290

Marjanska, M, Henry P-G, Ugurbil K, Gruetter R: Editing through Multiple Bonds: Threonine Detection. Magnetic Resonance Med. 2008; 59:245-251

Yacoub E, Uludag K, Ugurbil K, Harel N: Decreases in ADC observed in Tissue Areas During Activation in Cat Visual Cortex at 9.4T Using High Diffusion Sensitization. Magnetic Resonance Imaging 2008, In press

Muetzel R, Collins P, Mueller B, Schissel A, Lim K, Luciana M: The Development of Corpus Callosum Microstructure and Association with Bimanual Task Performance in Healthy Adolescents. Neuroimage 2008; 39(4):1918-1925 
Michaeli S, Sorce D, Garwood M: T2p and Tip Adiabatic Relaxations and Contrasts. Current Analytical Chemistry 2008, 4:8-25

Iltis I, Marjanska M, Du F, Koski D, Zhu X-H, Ugurbil K, Chen W, Henry P-G:

${ }^{1}$ H MRS in the Rat Brain Under Pentobarbital Anesthesia: Accurate Quantification of In Vivo Spectra in the Presence of Propylene Glycol. Magnetic Resonace Medicine 2008; In Press

Marjanska M, Henry P-G, Ugurbil K, Gruetter R: Editing Through Multiple Bonds: Threnonine Detection. Magnetic Resonance Medicine 2008; 59(2):245-251

\section{Presentations and Posters:}

Olman C, Van de Moortele P-F, Ugurbil K, Yacoub E: Retinotopic Mapping with 7 Tesla fMRI:

Improved Resolution and Decreased Experiment Duration. Society for Neuroscience November 2007

Iltis I, Koski D-M, Ugurbil K, Henry P-G: Single-Voxel1H MRS of the Rat Brain: Effect of Anesthesia. Joint Annual Meeting ESMRMB-ISMRM Berlin, Germany 2007

Iltis I, Koski D, Eberly L, Deelchand D, Valette J, Lim K, Ugurbil K, Henry P-G: Effects of PCP on the Prefrontal Cortex Metabolism in Intact, Anesthetized Rats. Joint Annual Meeting ESMRMB-ISMRM Berlin, Germany 2007

Muetzel R, Collins P, Mueller B, Lim K, Luciana M: The Development of Corpus Callosum Microstructure and Associations with Bimanual Task Performance in Healthy Adolescents. Cognitive Neuroscience Society's Annual Meeting 2007 


\author{
Project 22: Development Of Clinical Analysis Tools For MEG Data Gathered On Patients With \\ Epilepsy \\ Investigator: Michael P. Weisend, Ph.D. \\ Co-Investigators: Steve Stuffelbeam (MGH), Fa-Hsuan Lin (MGH) \\ Period of Report: 4/1/07-3/31/08
}

\begin{abstract}
:
An estimated 2.7 million Americans suffer from some form of epilepsy. Approximately $25 \%$ of this population, 6-700,000 individuals, does not achieve adequate seizure control with medication. These patients are said to have medically refractory or medically intractable epilepsy. Patients with medically refractory epilepsy can potentially cured by surgical resection of the epileptic brain tissue. These patients are, therefore, typically referred to comprehensive epilepsy centers where they undergo a series of inpatient and outpatient procedures as part of an evaluated for resective epilepsy surgery.
\end{abstract}

Magnetoencephalography (MEG) combined with high-spatial resolution magnetic resonance imaging (MRI) is a combination of techniques that can provide unique information about the precise locations of function and dysfunction, critical in epilepsy surgery planning (Knowlton et al., 2006; Papanicolaou et al., 2005). MEG is used to map the locations of epileptic activity in the brain. Eloquent cortex, serving functions such as movement, language, and memory, can also be spatially and temporal mapped with MEG. The result is a composite image that shows regions producing epileptic activity and their proximity to eloquent corteices. Importantly, no other neuroimaging technique is capable of producing maps of brain function and dysfunction with similar spatial and temporal resolution.

In spite of the unique capabilities of MEG whether it is a "standard of care" procedure during evaluation for epilepsy surgery is controversial. There are several reasons for this. First, MEG is rare with fewer than 20 MEG instruments used to evaluate epilepsy patients in the US. Second, clinical MEG scans are expensive as a result of the necessary capitol expenditure, maintenance, and staffing costs. Third, reimbursements for clinical MEG scans are difficult to obtain from health insurance companies as a result of somewhat arbitrary decisions labeling the technology as investigational. The chief reason for the labeling of MEG as an investigational, i.e. non-reimbursable, exam is that there have been no large-scale, multi-site studies demonstrating the utility of MEG in the evaluation for epilepsy surgery. Fourth, there is no standardization of hardware, software, or procedures for the clinical MEG exam across sites. Lastly, the analysis of clinical MEG data is incredibly time consuming as a direct result of the lack of standard data analysis tools. The MRN Institute is uniquely positioned to address all of these problems.

The MRN Institute and its partner sites have a common interest in using MEG for clinical service as a potentially important revenue stream and multiple MEG machines from different manufacturers. Further, The MRN MEG consortium studies have developed tools that allow a standardized, machine independent analysis of MEG data. In this project we aim to build upon the data pooling capabilities by application of these tools to the real world problem of epilepsy. Investigators from The MRN Institute and Massachusetts General Hospital will work toward the creation of a set of standard examination procedures, data analysis tools, and reporting formats for clinical MEG data from patients with epilepsy. Together these efforts will make possible the time efficient, large-scale, multi-site studies that can definitively evaluate the utility of MEG for patients with epilepsy.

\title{
Objectives and Findings:
}

Different institutions have different MEG instruments and these hardware differences are immutable. However, different institutions can optimize the clinical exams for epilepsy that are performed at each site 
to improve clinical care and increase the number of patients who benefit. the The objectives of this proposal were to standardize the MEG acquisition in epilepsy including the sampling rates, filter settings, time parameters. In addition, our goal was to reduce the heterogeneity in the collection of simultaneous EEG. We had planned for technical and scientific staff from the MRN Institute will travel to MGH and observe the clinical epilepsy exam. Personnel from MGH will also travel to MRN for observation of the clinical epilepsy exam. This would have allowed us to minimize the intangible differences between exams and increase standardization of analyses for clinical epilepsy data.

The differences between the Mind and MGH sites are significant, but not insurmountable. The different MEG instruments at MRN and MGH have different data formats, different MRI coregistration schemes, different source localization routines, and different data preprocessing schemes. Previous MRN collaborations with MGH have laid the groundwork for this partnership with software that can import the two datasets into a standard format. The task that remains is to standardize a set of analysis routines. We intended to hold biweekly meetings scientific staff at MRN and MGH will collaborate with a programmer create and implement a set of standard data analysis routines for spike detection, head modeling, coregistration with MRI, source localization procedures, and a common clinical reporting format will be developed. Unfortunately, the MGH portion of the funding for this study was cut at the administrative level. The Mind personnel continued with the goals of this project unilaterally.

While the MEG machine at MRN is primarily a research instrument, MRN has sought to develop a program to examine as a potential funding stream to insure future sustainability. Five patients with epilepsy were examined at MRN. The data from these patients served as a test bed for the tools that were developed to examine the brains of patients with epilepsy. Tools developed as part of the MEG Technology program and supplemental tools developed at MRN for analysis of epilepsy data were used to examine the data from each person with epilepsy. One critical step in the analysis of epilepsy data is the detection of interictal epileptiform events called spikes. Scientists at MRN have developed a method to accelerate detection of spikes (see Figure 1). When a spike has been located it is crucial to accurately model the brain activity giving rise to the spike. This involves segmentation of the brain surface from MRI, coregistration of the MEG and MRI data, generation

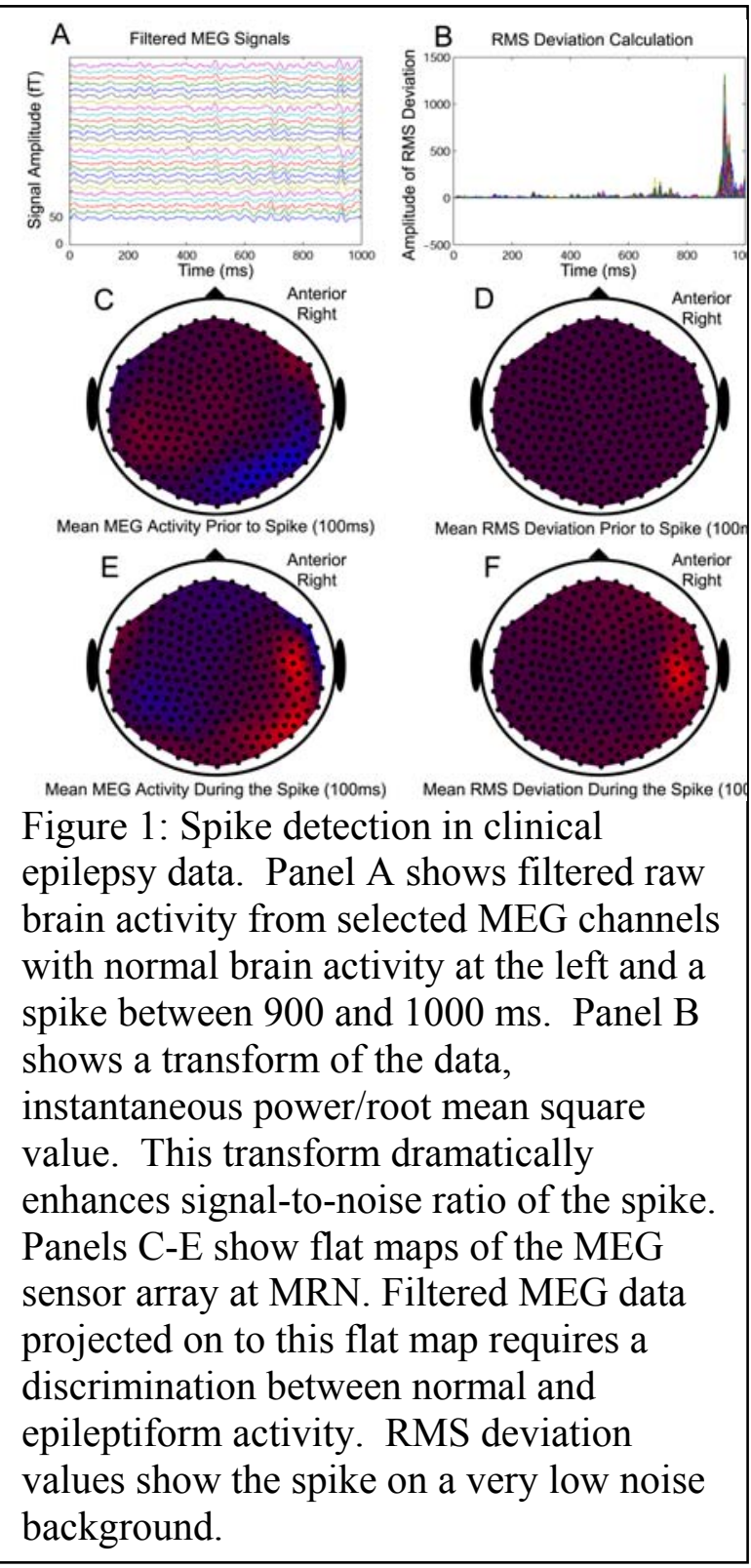
of head models, choosing an epoch over which to model the brain activity and choosing the number of sources to include in modeling the activity (spikes can come from single generators or multiple generators), and displaying the results on the brain. Often the spikes propagate from one region of the brain to another. In this case it is critical to interrogate the data with the intention of determining the 
location where the epileptiform activity originates (Iwasaki et al., 2002). The spike finding algorithm illustrated has been integrated into a larger tool for examining raw data Figure 2 . The tool illustrated in Figure 2 enables scanning of data across all time points in the recording as well as jumping from epoch to epoch that contain candidate spikes. When a spike is detected in the MEG recording and identified in the display

These cross site cross platform tools will enable the standardization of epilepsy analyses across sites. We are currently working on standardization of the output format for reports with Dr. Bruce Fisch in the department of Neurology at the University of New Mexico. A few political and administrative hurdles remain, but the MRN will soon be able to apply the techniques developed here to bettering the health of subjects with epilepsy. Moreover, the data analysis will become highly efficient with respect to time and training. For example, a typical clinical analysis can take from most of a day to several days. This is the

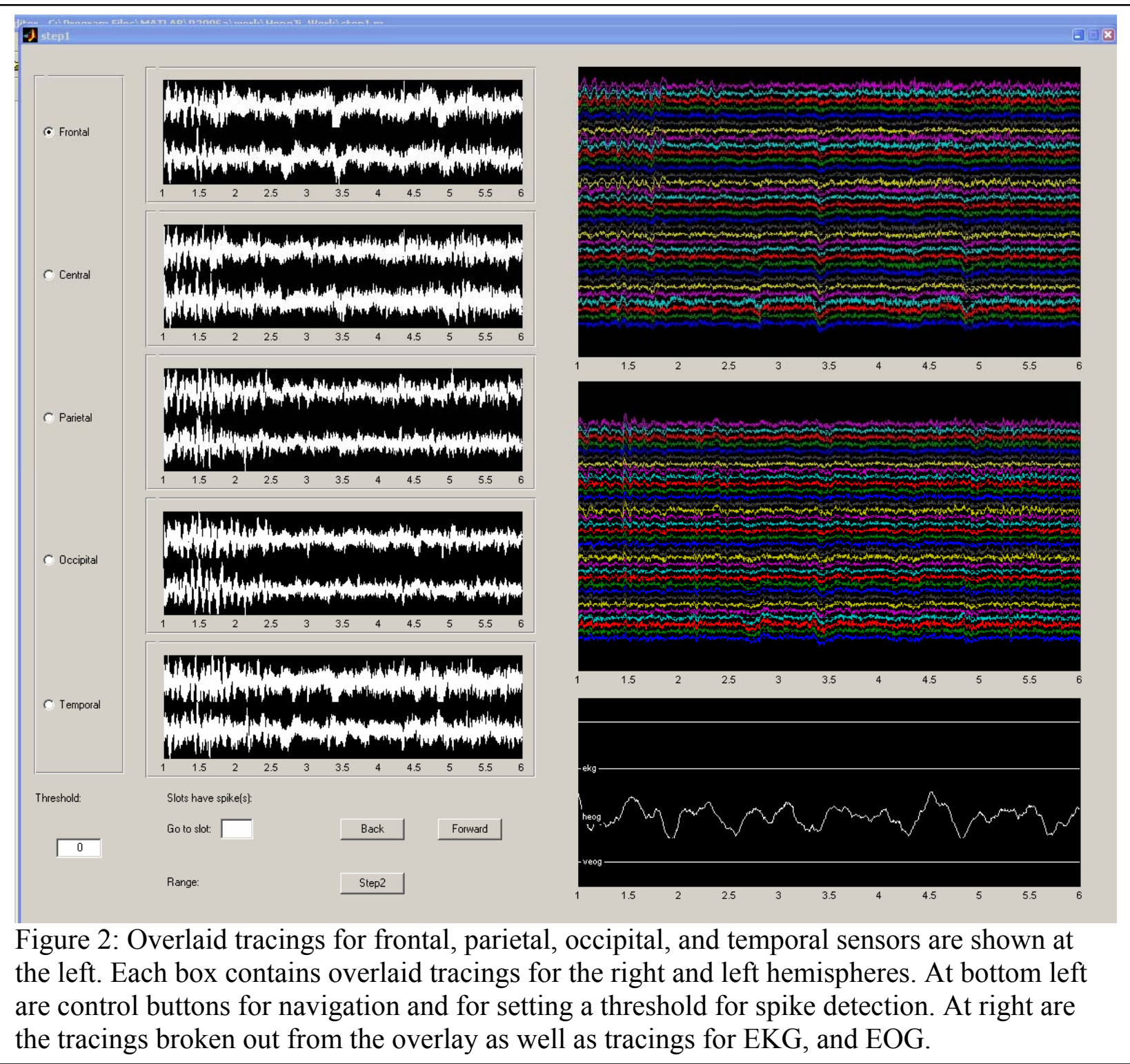




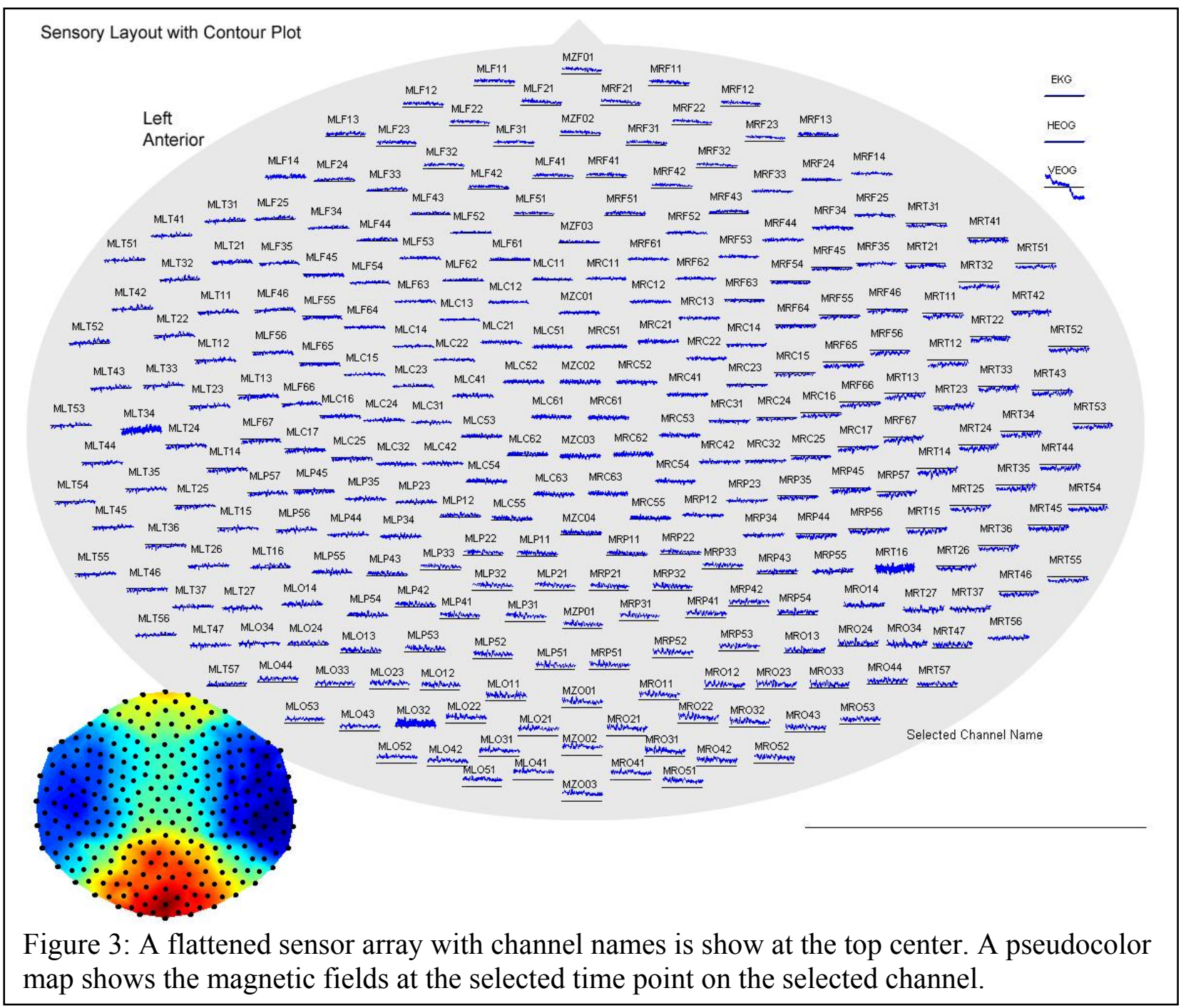

shown in Figure 2, the data if forwarded to the display in Figure 3 that shows the layout of the channels in a flattened sensor array (Figure 3). If the data displayed in Figure 3 is thought to show an epileptiform spike, the data is forwarded to the MNE localization display. The automation and integration of these tools is nearly complete. The MRN Institute scientists are poised to make giant leap forward in the analysis of clinical epilepsy data

result of the complexity of the clinical analysis. The vast majority of the work is completed by hand, but could be automated. This would make the analyses far more time efficient. At the current time patients must sometimes wait inordinate amounts of time, weeks, for the results of an MEG exam. Furthermore, the lack of standardized tools and exam techniques make retraining necessary if a scientist moves to a new site and makes getting a second opinion about the results of an exam nearly impossible. These problems will be addressed directly by the application of MIND tools to analysis of clinical epilepsy data recorded with MEG6. A development that is long overdue in the use of MEG as a clinical diagnostic technology. 


\section{Project 23: Magnetic Resonance (MR) Core \\ Investigator: Vince Calhoun, Ph.D. \\ Period of Report: 4/1/07-3/31/08}

\section{Abstract:}

The MR Core provides access to state-of-the-art magnetic resonance imaging equipment for MIND investigators and the UNM community. In addition, we coordinate with our partner sites in advancing the important technological issues involved in the use of imaging information as a diagnostic tool.

The MR Core provides access to two MR systems including:

- Siemens 3T Trio with Total Imaging Matrix (TIM) Application Suite: The Trio 32-channel system represents state of the art in MRI hardware. It is capable of BOLD EPI, diffusion tensor imaging, perfusion and diffusion imaging, and spectroscopy. With 32 usable receiver channels as standard, the system allows for the use of current phased array coils (from 4 to 16) to improve sensitivity and speed of acquisition and is ready for future coil designs with more than 16 elements. This system is interfaced to an MR-compatible patient monitoring unit featuring pulsed oximetry, heart rate monitor, $\mathrm{O}_{2}, \mathrm{CO}_{2}, \mathrm{NO}$, and halothane measurement, blood pressure monitor and EKG (OmniTrak, InVivo Research, Inc. Orlando, FL).

- Siemens Sonata Maestro 1.5 Tesla MRI Scanner: The system is equipped with standard transmit and receive RF coils, as well as receive-only surface coils. It is capable of BOLD EPI, diffusion tensor imaging, perfusion and diffusion imaging, and spectroscopy. This scanner is interfaced to an MRcompatible patient monitoring unit featuring pulsed oximetry, heart rate monitor, $\mathrm{O} 2, \mathrm{CO} 2, \mathrm{NO}$ and halothane measurement, blood pressure and EKG (OmniTrak, In Vivo Research, Inc. Orlando, FL). TurboFIRE real-time data analysis software is directly interfaced to the scanner operating system.

\section{Objectives and Findings:}

1. Installation of the $3 \mathrm{~T}$ Trio scanner including adjustment and installation of custom pulse sequences and reconstruction programs.

The scanner is operational with all pulse sequences available and in use in multiple studies. Currently 17 funded studies and multiple external funded studies are using the scanner.

2. Standardization of quality assurance process, protocols on both scanners and integration of the scanner data into the neuroinformatics database and image analysis pipelines: In conjunction with then neuroinformatics core, we are developing a completely automated pipeline for the scanner data

We have automated e-mails which are submitted to each user of the 3T scanner indicating summaries of which studies which were performed each data. In addition a fully automated pipeline functioning for all funded studies mentioned above. This includes automatic fMRI analysis, DTI analysis (computing FA maps and tract tracing inputs), VBM analyses (native and spatially normalized segmented images), and Freesurfer analysis.

3. Provision of scans for DOE funded investigators in order to generate additional external grants and accomplish the goals of the projects.

We have provided through a pilot scan committee seed scans to multiple investigators at MIND. Several of these have already led to funded studies.

4. Testing of a 32-channel coil in conjunction with Larry Wald at the MGH partner site 
Due to budgetary limitations we have not implemented the 32-channel coil but are still in discussion with Larry Wald and would like to do this if funding permits.

5. Establish contracts with UNMH and community agencies to perform clinical MR scans and generate additional program revenue

Clinical studies from UNMH generated approximately $\$ 500 \mathrm{~K}$ last year. We continue to pursue this relationship.

\title{
Project 24: MIND Mobile Imaging Core Investigator: Kent Kiehl, Ph.D. Period of Report: 4/1/07-3/31/08
}

\begin{abstract}
:
The MIND Mobile Imaging Core is dedicated to using state-of-the-art brain imaging techniques to delineate the neurocognitive architecture underlying some of society's most enigmatic and costly mental health disorders. These disorders include alcohol and substance abuse and criminal psychopathy (i.e., psychopathic personality). These latter mental health disorders are commonly associated with severe and repetitive criminal behavior that leads to incarceration. The MIND Mobile Imaging system is the first of its kind that will be deployed to incarcerated facilities to study these populations in large numbers. The goal of the MIND Mobile Imaging Core is to develop a better understanding of the neurobiology of psychopathy and related mental health disorders so that we may develop effective treatments for these conditions. The MIND Mobile Core may also be deployed to other remote populations. For example, preliminary discussions are underway with the US military to study soldiers before active deployment to see if we can use brain imaging to predict which individuals are susceptible to Post Traumatic Stress Disorder (PTSD). The Mobile system would be deployed directly to the military base and hundreds of soldiers could be imaged in a very short time. A brain based test predicting susceptibility to PTSD could dramatically improve the quality of life for many active duty personnel.
\end{abstract}

The MIND Mobile Imaging system is comprised of a Siemens' 1.5T Avanto Magnetic Resonance Imaging system and a 72-channel high-resolution EEG/ERP Biosemi system. The Avanto is the most advanced $1.5 \mathrm{~T}$ system in the Siemens product line. The Avanto is a ultra-short $150 \mathrm{~cm}$ (4'11') long, whole body superconductive $1.5 \mathrm{~T}$ magnet with 5 th generation active shielding (AS) technology with counter coils, External Interference Shielding (E.I.S.) and excellent homogeneity (based on 24 plane plot, $50 \mathrm{~cm}$ DSV type. $0.8 \mathrm{ppm}$ ). The system comes equipped with a 12-element Matrix head coil capable of ultra-fast parallel acquisition in either 4-channel (CP Mode), 8-channel (Dual Mode) or 12-channel (Triple Mode) settings. The SQ-engine Gradient System with AudioComfort was selected. The SQengine gradients have a maximum amplitude of $45 \mathrm{mT} / \mathrm{m}$ for the longitudinal direction and $40 \mathrm{mT} / \mathrm{m}$ for horizontal and vertical direction. The gradient slew rate is $200 \mathrm{~T} / \mathrm{m} / \mathrm{s}$ with a minimal rise time of $200 \mu \mathrm{s}$ (from 0-40 $\mathrm{mT} / \mathrm{m}$ amplitude). AudioComfort is an acoustic noise buffer that leads to a $30 \mathrm{~dB}$ (A) attenuation of gradient noise compared to other conventional systems that leads to a reduction of $97 \%$ in sound pressure. This latter sound reduction is of great benefit to functional imaging studies. The MR system comes completely integrated into a trailer (see Figure 1). The system trailer will also house an EEG/ERP data collection system for brain potential studies. 


\section{Objectives and Findings:}

The mobile system was delivered to the MIND Institute on February 26, 2007. During the subsequent six weeks following delivery, custom auditory and visual presentation capabilities for functional magnetic resonance imaging were installed. The mobile trailer was custom designed with a special projector housing, multiple waveguides for passing fiber optic response devices from the control room to the MR room, and the control room was split into two rooms - one for MR data collection and the other for EEG data collection. The design also provided an interface with the Siemen's MR console to pass all the raw imaging data to an offline computer for processing.

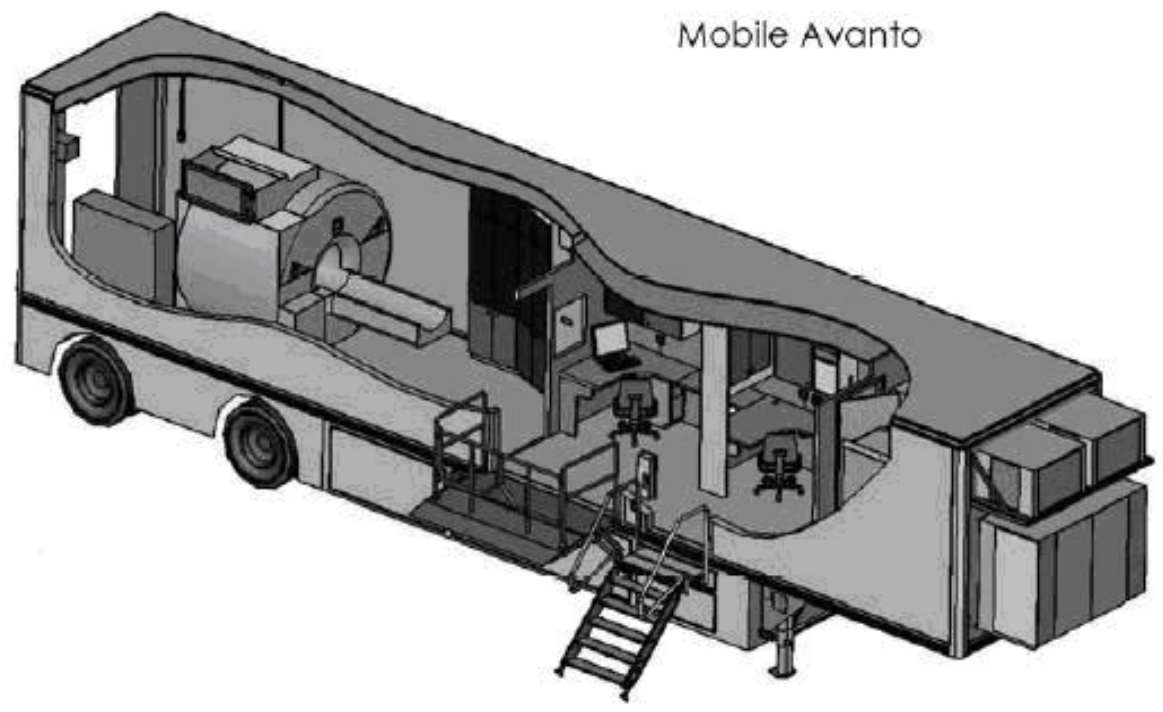

Figure 1. Graphical representation of the mobile imaging laboratory (provided by Medical Coaches, Inc.).

Separate from the technical requirements of making the mobile system operational, there were several administrative tasks that required attention, including a trailer inspection, insurance, license, and registration. The Mobile system was deployed on April 7, 2007, with a first stop to the Western Men's Prison in Grants, NM.

In our 2007 proposal, we indicated that there were three major NIH grants that would be utilizing the mobile imaging scanner. As of February 15, 2007, we had completed over 700 scans on 300 inmates (many inmates participate in longitudinal research) across three studies. Below is a progress report for each activity.

\section{Project Title: ERPs and fMRI of Emotion and Cognition in Psychopathy}

Funding: NIMH R01 MH0705539-01 (12/01/04-11/30/08; no cost extension planned through 2009 due to grant relocation from IOL/Yale University to MIND Institute). Renewal planned to be submitted end of 2008.

Kiehl, K.A., Calhoun, V.D., \& Stevens, M.C.

Project Summary: The purpose of this project is to test the paralimbic hypothesis of psychopathy (Kiehl, 2006) in populations of ethically diverse males and females from varied socio-economic backgrounds. High temporal resolution electrophysiological techniques and high spatial resolution hemodynamic imaging will be used to map the functional neural architecture associated with affective, error-monitoring, 
and salient stimulus processing tasks in groups stratified by psychopathy scores. The ultimate aim of the proposed project is to obtain a more complete understanding of the brain systems implicated in psychopathy, and secondarily, to begin to understand how brain function relates to symptom profiles in psychopathy (i.e., the emotional detachment verses impulsive lifestyle). The data will permit an examination of psychopathy-specific paralimbic brain function abnormalities along gender and socioeconomic lines, exploration of how brain function abnormalities relate to psychopathy symptom subtypes, and provision of data on the inter-relationship of functional brain abnormalities in psychopathy and alcohol and substance disorders. This is a large scale series of studies that plan to enroll more than 300 (75 psychopaths) participants who complete two scan sessions per subject.

Progress made to date: This study collected data in 150 participants in Connecticut before moving to The MIND Institute. The participants in Connecticut were drawn from community sources, including individuals currently receiving substance abuse treatment in residential programs or individuals on probation or parole. Preliminary results are supporting research hypotheses although we have had difficulty finding individuals with high scores on psychopathy. Utilizing correlational analyses of the 100 clinical subjects, we have shown that psychopathy scores are negatively correlated with the amplitude of the hemodynamic response in paralimbic brain regions during performance of attention and orienting tasks, as well as during error monitoring tasks. In New Mexico we have obtained IRB approval from the University of New Mexico as well as federal approval from OHRP and local prisons where we are recruiting research participants. To date we have consented more than 80 inmates and completed two scans on more than 75 inmates. Twenty inmates have met clinical criteria for psychopathy ( $\sim 25 \%$ of sample to date). Data processing is ongoing.

Utilization and benefit of the mobile imaging system: The mobile imaging system will allow collection of imaging data in prison samples with much higher rates of psychopathy than that observed in the community. We have found very few individuals who have met criteria for psychopathy in the community but in our first three months of prison research since relocating to New Mexico we have found that $25 \%$ of the prison sample (from more than 200 inmates interviewed) meet criteria for psychopathy. Thus the mobile MRI system is facilitating research in a population that so far proved very difficult (to nearly impossible) to study in sufficient numbers in other settings. It is estimated that this project would utilize approximately $10 \%$ of the mobile system usage in the first three years ( 75 inmates/controls scanned twice in this protocol per year). We hope to renew this project in 2009 for another five years. Both healthy controls and incarcerated participants would be collected.

Preliminary functional MRI data from the mobile MRI system from the adult male prison in New Mexico.

Figure 3: Results from the mobile MRI scanner of inmates $(\mathrm{n}=25)$ during commission of an error during a Go/No Go study ( $\mathrm{p}<$ .001). Areas associated with error-monitoring include rostral and caudal anterior cingulate, thalamus, posterior cingulate, fusiform, and parietal cortex - all regions where we have shown similar results in prior studies. These data illustrate that the mobile MRI system is capable of collecting robust fMRI data.

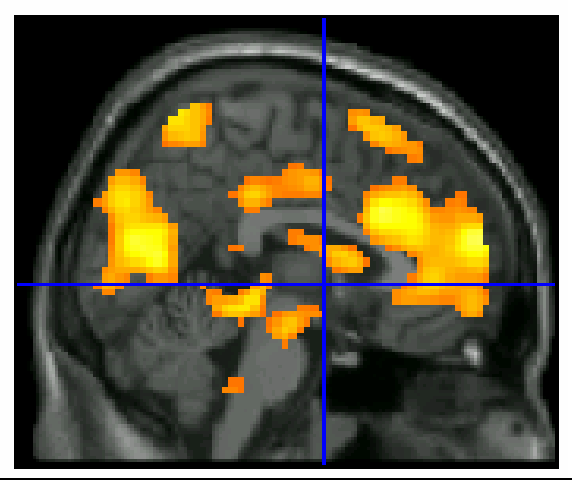


Figure 4: Results from the mobile MRI scanner of inmates $(n=54)$ during target detection of an auditory 'oddball' task ( $\mathrm{p}<$ $.001)$. These data replicate previous results from our laboratory (Kiehl et al., 2005) and illustrate that the mobile imaging system is capable of collecting clean data in small, susceptibility-prone areas (i.e., right amygdala).

We have also been collecting Diffusion Tensor Imaging (DTI) data on the mobile MRI system. DTI is based on random Brownian motion of water molecules which preferentially diffuse along rather than across axonal fascicles. Diffusion tensor, which can be measured by MRI, describes this anisotropy of motion. The diffusion contrast of an MRI signal depends on white matter micro-structures, such as myelin density, axonal damage, density, size of axons, and other axonal properties. Diffusion measurement by MRI is a macroscopic measurement of microscopic white matter properties. Assuming that the direction of largest diffusion corresponds to the direction of axon fiber bundles, DTI can used for tracking fiber bundles (DTI-FT) by concatenating fiber directions in adjacent voxels like flow streamlines.

DTI experiments are being conducted on the mobile Siemens Avanto 1.5T scanner with a 12-channel phased array head coil. The data is collected in the axial direction along the AC-PC line with a FOV $=256 \times 256 \mathrm{~mm}$ with a $2 \mathrm{~mm}$ slice thickness, 72 slices, $128 \times 128$ matrix size, $\mathrm{TE}=82 \mathrm{~ms}, \mathrm{TR}=9000 \mathrm{~ms}$, $\mathrm{NEX}=1$ and with a GRAPPA acceleration factor of 2 . The DTI sequence has 30 directions, $b=800 \mathrm{~s} / \mathrm{mm}^{2}$, and the scan with $b=0$ is repeated five times for optimal signal-to-noise ratio. The 35 diffusion measurements $(30+5)$ have been organized in five groups of seven, such that each group when included with the previous groups makes an optimal set for calculating the diffusion tensor from those measurements. This strategy ensures that if the subject moves then all the previous measurements can be optimally used for diffusion tensor calculations. The sequence is repeated twice and the data combined to improve SNR. The total experiment time is 12 minutes.

The data processing streamline consists of a) motion and eddy current correction (Flirt in FSL), b) rotation of gradient directions to adjust for image orientation (IDL routine), c) calculation of the diffusion tensor and other DTI parameters (Dtifit in FSL), d) registration of T1 and the DTI images to the Montreal Neurological Institute (MNI) space.

An example of the color coded FA image collected from the mobile 1.5T scanner is shown below. Colors are coded based on the direction of the fibers (red is left/right; green is superior/inferior; blue is anterior/posterior). These data are being collected as part of every project on the mobile MRI. Group analysis will be done to extract correlations with neuropsychological and psychometric test parameters. 

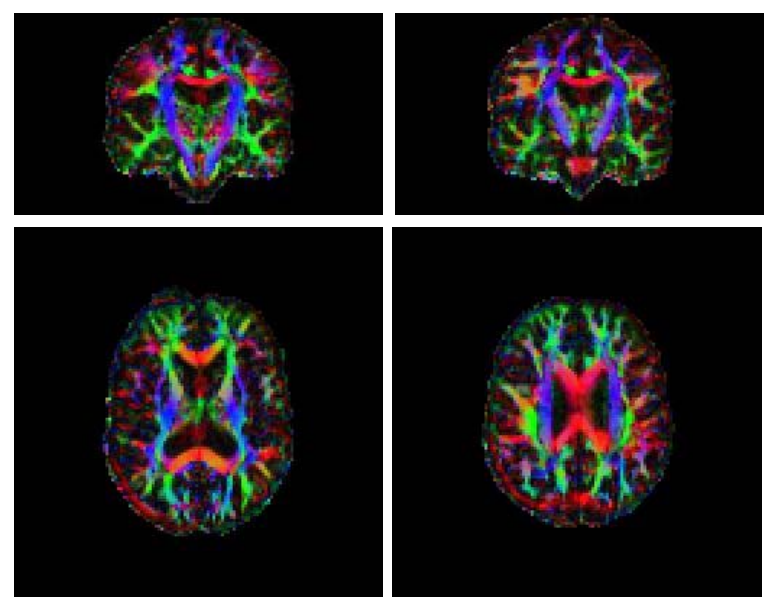

These are the first DTI data that have been collected on individuals with psychopathy.

Project title: Neurocognitive Assessment of 'Callous' Conduct Disordered Youth

Funding: NIMH 1 R01 MH071896-01 (12/01/05-11/30/10)

Kiehl, K.A., Calhoun, V.D., \& Kazdin, A.

Project summary: Recent research has identified a callous and unemotional subtype of Conduct Disorder (CD) in adolescence that closely resembles adult conceptualizations of psychopathy. Accumulating evidence suggests that the affective and interpersonal impairments in Callous CD are measurable at an early age and may persist into adulthood. It is well known that adult psychopaths, relative to nonpsychopaths, are responsible for a disproportionate amount of social disruption, both civil and criminal. It is thus potentially important to develop a better understanding of the adolescent precursors to adult psychopathy. Recent electrophysiological and hemodynamic imaging studies completed by the primary investigator suggest that adult psychopathy is associated with neurocognitive abnormalities in the paralimbic system during performance of affective, error monitoring, and salient stimulus processing tasks. The primary paralimbic structures that appear to be implicated include the amygdala, orbital frontal cortex, anterior superior temporal gyrus, and anterior and posterior cingulate. The purpose of the present proposal is to test the hypothesis that Callous CD in adolescence is associated with functional abnormalities in the paralimbic system. It is hypothesized that paralimbic dysfunction will characterize adolescents with Callous CD features and differentiate these youth from peers without such features. It is also hypothesized that Callous CD will be associated with alterations in the normal developmental trajectories associated with processing affective, salient, and error stimuli. High temporal resolution electrophysiological techniques and high spatial resolution hemodynamic imaging will be used to describe the functional neural architecture associated with affective, error monitoring, and salient stimulus processing tasks in groups stratified by Callous CD scores. The ultimate aim of the proposed project is to obtain a more complete understanding of the brain systems implicated in CD adolescents characterized by high callousness, and secondarily, to begin to understand how the function of the paralimbic system relates to other disruptive disorders commonly diagnosed in adolescence. At the conclusion of the study, data will be available to characterize the functional neuroanatomy in youth with Callous CD to determine what, if any, neurocognitive patterns may contribute to the persistence of antisocial and "psychopathiclike" features. The data also will delineate the relationships between the function of the paralimbic system and the symptom subtypes of Callous CD.

Progress made to date: We evaluated 114 youth (99 with neurocognitive data; 68 with disruptive behavior disorders) who fit study inclusion criteria. These adolescents were recruited from community sources of 
individuals on probation for criminal offences and referral from local clinicians. Consistent with hypotheses, high PCL-YV scores (i.e., youth scores on the psychopathy measure) showed an inverse relationship with the amplitude of the hemodynamic response in the anterior cingulate during errormonitoring. In addition, on an emotional lexical decision task, higher PCL-YV scores were associated with reduced hemodynamic activity in paralimbic areas including amygdala and posterior cingulate. These results suggest that the study hypotheses will be supported. However, community samples have proven difficult to find adolescents who score in the clinical range on the PCL-YV measure of adolescent psychopathy or callous conduct disorder. We only found $5 \%$ of our sample met clinical criteria for adolescent psychopathy (aka, Callous conduct disorder).

The project was moved from Connecticut to New Mexico on January 1, 2007. In New Mexico we have obtained IRB approval from the University of New Mexico, federal approval from OHRP and local prisons where we are recruiting adolescent research participants. The mobile MRI system was deployed to a maximum security juvenile facility in Albuquerque, NM, from June 15-August 1, 2007. This 140 bed facility is populated by the most severe adolescent violent and sex offenders in New Mexico. Eighty incarcerated adolescents were scanned twice in this protocol. The rates of adolescent psychopathy (callous conduct disorder) have been $22 \%$ in this population. This data is currently being analyzed and written up for publication.

Utilization and benefit of the mobile imaging system: The mobile imaging system has allowed us to collect an unprecedented number of incarcerated adolescents in only six weeks $(n=80)$. To our knowledge, this is the very first fMRI data in adolescent maximum security offenders, and this research would not have been possible without the mobile MRI system.

This effort provided excellent pilot data for several NIH operating grant proposals. Indeed, Dr. Bryan recently submitted an NIH grant (see Pending Grant Applications Appendix) in which a major component of the grant is to use the mobile MRI to examine decision making processes in adolescents. This grant was well received with a first round score of 120 (2\%ile).

It is estimated that the current project (NIMH 1 R01 MH071896-01) would utilize approximately 10\% (160 total sessions or 40 participants scanned once from four groups (incarcerated: high psychopathy $(n=40)$, medium psychopathy $(n=40)$, and low psychopathy $(n=40)$; community: low psychopathy controls $(n=40))$.

Figure 5: Results from the mobile MRI scanner of adolescent incarcerated inmates $(\mathrm{n}=35)$ during commission of an error during a Go/No Go study $(\mathrm{p}<.001)$. Areas associated with error-monitoring include rostral and caudal anterior cingulate, thalamus, posterior cingulate, fusiform, and parietal cortex - all regions where we have shown similar results in prior studies (Kiehl et al., 2000)

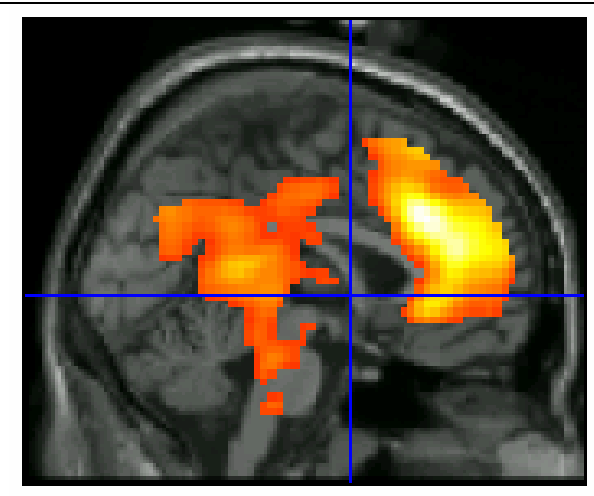


Project title: Neurocognitive changes associated with behavioral treatment in cocaine abusers

Funding: NIDA; 1 R01 DA020870-01 (10/01/05-09/30/10)

Kiehl, K.A., Calhoun, V.D., Clark, V., Wilber, C., Jaffe, A., \& Pearlson, G.D.

Project summary: Heavy cocaine users who repeatedly fail in treatment account for a disproportionately large share of cocaine consumption and associated demand of mental health and criminal justice resources. We have recently developed a tailored cognitive behavioral protocol, Substance Expectation Therapy, for the treatment of cocaine abuse. In a large study ( $\mathrm{n}=157)$, Substance Expectation Therapy was compared to Relapse Prevention and Addiction Counseling for the treatment of cocaine abuse (NIDA 5 RO1 DA11249-02; PI Jaffe). Substance Expectation Therapy was found to be superior to Relapse Prevention and Addiction Counseling for preventing relapse in cocaine abusers. A major aim of this proposal is to replicate the efficacy of Substance Expectancy Therapy for the treatment of criminal justice involved cocaine abusers. A second major aim of this proposal is to build upon research that has shown abnormalities in the brain's response to distracting stimuli predicts relapse in cocaine abusers (Bauer, 1997). We will use multimodal brain imaging, combined with advanced image processing techniques including Independent Component Analyses, to further delineate the neurocognitive systems that convey risk for relapse in cocaine abusers. In addition, this proposal will track changes in neurocognitive systems associated with response inhibition, deviance and novelty detection, and salient stimulus processing across the three treatment protocols. Our primary hypotheses are that Substance Expectancy Therapy will be superior to Relapse Prevention and Addiction Counseling for preventing relapse in cocaine abusers. It is also hypothesized that multimodal, compared to unimodal, brain imaging will convey superior predictive power for assessing relapse in cocaine abusers. Finally, we hypothesize that Substance Expectancy Therapy will show increased modulation in the brain systems associated with inhibitory processing and salient stimulus processing over the course of the 12 week treatment protocol. In summary, the results of this proposal will test the efficacy of Substance Expectancy Therapy compared to Relapse Prevention and Addiction Counseling, will further delineate the functional architecture underlying risk for relapse in cocaine abusers, and will examine neuronal changes associated with tailored cognitive-behavioral therapy for cocaine abuse.

Progress made to date: This study was halted in 7/2006 (before treatment began) in order to transfer the project from Connecticut to the MIND Institute. We are projected to run a no-cost extension into 2011. This project was slated to work with inmates in a residential (locked down at night) facility on the Institute of Living campus (where the PI's former employer, Olin Neuropsychiatry Research Center is located). These residential drug abusers are serving alternative placement instead of prison. However, the sample size available for study was relatively small (about 80 per year) whereas in the prisons of New Mexico we have access to more than 7500 inmates with substance abuse problems - provided the mobile MRI system is available for deployment. In New Mexico we have obtained IRB approval from the University of New Mexico as well as federal approval from OHRP and local prisons where we are recruiting research participants. We have recruited, hired, and trained a new program manger (Dr. Sanjuan), two new research assistants, and nine new therapists. We have also completed updating the training materials (including revisions of all three treatment manuals), organized psychiatric assessment materials, set up six interview and therapy rooms at the prison, equipped research assistants and therapists with new tablet computers and video recording equipment, and developed a computer database for data input, storage, and analyses. In three months, we have enrolled 45 substance abusers in therapy. The imaging component of this study contains a longitudinal design and all enrolled inmates have completed baseline testing as of 9/15/07. The mobile MRI is performing flawlessly and we are working towards our midpoint of therapy when additional imaging data is to be collected.

Utilization and benefit of the mobile imaging system: More than $75 \%$ of inmates in New Mexico $65 \%$ nationwide) meet criteria for alcohol and/or substance abuse/dependence. Substance abuse treatment and research with incarcerated samples is one of NIDA's major new initiatives (Volkow, 2004, Volkow and 
$\mathrm{Li}, 2004)$. The mobile imaging system is critical for working with this underrepresented population. This imaging system permits access to more than 7500 inmates (of 10,000 in the state). Thus, the imaging system will facilitate data collection and most importantly, permits us to conduct neuroscience research with a very underrepresented population. We project between $15-25 \%$ mobile MRI usage for this protocol which requires five fMRI sessions per participant (two at baseline, one at six weeks after therapy onset, and a final exam following completion of therapy at 12 weeks). We anticipate 50-75 inmates per year or 250-375 scan sessions per year.

\author{
Project 25: Magnetoencephalography and Electroencephalography Core \\ Investigator: Michael Weisend, Ph.D. \\ Period of Report: 4/1/07-3/31/08
}

\begin{abstract}
:
Magnetoencephalography (MEG) is a non-invasive functional neuroimaging technique that records the magnetic fields generated by the brain. When used in combination with magnetic resonance imaging (MRI), the locations and time courses of activity in the brain can be specified on scales of millimeters and milliseconds. As a functional imaging modality MEG is a highly specialized technique that is conducted at approximately 100 sites world wide. MEG has an advantage in spatial resolution over electroencephalography (EEG), and greater temporal resolution than functional MRI (fMRI), positron emission tomography (PET), or single photon emission computed tomography (SPECT). The unique information provided by MEG has basic research, clinical research, and clinical diagnostic applications. The MEG program at the Mind Research Network is critical to the performance of multiple externally funded grants with a total Value, including indirect costs, of more than $\$ 6.4$ million.
\end{abstract}

As of this writing, the MEG instrument at the MIND provides services for six MIND scientists and four external scientists. Eight of the ten referenced, including all six MIND scientists have extramural funding to support their MEG efforts. This figure is up from two extra- murally funded MIND scientists last year. The MEG operates at roughly $60 \%$ capacity during standard working hours. Bi-weekly MEG users group meetings are organized by the MEG/EEG Program personnel. In these meetings the PIs and their staff are kept abreast of any changes to MEG/EEG Program policies and procedures. Input from the MEG users is also solicited in an effort to improve the service of the MEG/EEG Program. MEG Users Group meetings are an excellent forum for facilitating collaboration among PIs and staff members utilizing MEG/EEG Program services. The MEG/EEG Program area also provides support for the babySQUID ${ }^{\circledR}$. The babySQUID ${ }^{\circledR}$ is a one of a kind device that was designed and built by Dr. Okada and Tristan Technologies, Inc. It was designed specifically for MEG measurements of infants and toddlers. The system has $76 \mathrm{MEG}$ channels laid out in a hemispherical design that allows the baby to sleep while resting their head on the Dewar. The babySQUID system is a mobile unit. This allows the system to be moved into the MIND Institute's magnetically shielded chamber that houses the adult VSM 275 channel MEG system for data collection. These facilities continue to be instrumental in the maintenance of active collaborations with researchers at MEG labs at University of California San Diego, the National Institutes of Health, Massachusetts General Hospital, Los Alamos National Laboratory, University of New Mexico Departments of Psychology, Radiology, and Psychiatry, and the New Mexico VA Health Care System Department of Psychiatry. 
The MEG/EEG Program also provides support and services to Dr. Kiehl as he begins collecting data for his R-01 on schizophrenia and psychotic bipolar disorder. Accumulating evidence suggests that a cardinal abnormality in schizophrenia is aberrant connections between brain regions (i.e., functional connectivity). Aberrant functional connectivity has been observed in frontal and temporal lobes in schizophrenia during performance of target detection of low probability events. Patients with bipolar illness show impairments in multiple frontal circuits, basal ganglia, parietal cortex and cerebellum during target detection but show greater activity in temporal lobe regions compared to patients with schizophrenia. These results suggest psychotic bipolar illness may be associated with aberrant functional connectivity between frontal, striatal, and temporal lobe circuits - but in a pattern opposite to that observed in schizophrenia. The present proposal seeks to test the hypothesis that event-related potential (ERP) topography, patterns of hemodynamic activity, and measures of functional connectivity, will reliably differentiate patients with schizophrenia from patients with psychotic bipolar illness during performance of target detection. High temporal resolution electrophysiological techniques and high spatial resolution hemodynamic imaging will be used to map the functional neural architecture associated with target detection. Independent Component Analyses will be used to examine the patterns of aberrant functional connectivity. Patients with clear diagnoses of schizophrenia or psychotic bipolar illness will be studied during the first week of a relapsing psychotic episode and again at 3 months and at 6 months post-episode. The longitudinal design will permit evaluation of state and trait markers of the two disorders. It is hypothesized that patients with schizophrenia will be reliably differentiated from patients with psychotic bipolar illness based on the pattern of hemodynamics and functional connectivity measures in frontal, temporal and subcortical structures. This work will be an important step in developing procedures to aid in the differential diagnosis of schizophrenia or psychotic bipolar illness at early stages of the disorder. The data also will permit an examination of psychotic symptoms (i.e., disorganization, reality distortion, and psychomotor poverty) associated with electrophysiological and hemodynamic measures of brain function.

\section{Objectives and Findings:}

1. MIND MEG/EEG Program personnel will work with the MEG users group and collaborate with experts in signal processing to automate, to the greatest extent possible, the analysis of MEG data.

2. MIND MEG/EEG Program personnel will develop a funding base from clinical research, including drug trials, and clinical diagnostic services, including presurgical mapping of function and dysfunction in patients with epilepsy, brain tumors, and arteroveinous malformations.

With respect to Objective 1, two of three large pieces of the automated data processing scheme are finished and a third is partially complete. The automation of MEG data preprocessing; including artifact rejection, filtering, and creation of time locked averages; is complete. The calculation of time-frequency representations is also fully automated. The automation of source localization with MNE is partially completed. The automation of source localization is scheduled to be complete on April $1^{\text {st }}, 2008$. John Mosher from Los Alamos National Laboratory has been heavily consulted in these automation processes. Vince Calhoun from MIND will be involved in the implementation of these automation routines on the local MIND network. They are currently limited to a few machines that are routinely used for MEG data analyses.

Concerning Objective 2, the search for revenue streams for clinical research is also well underway. Dr. Weisend has programmed data analysis tools for processing clinical epilepsy data and they are ready to be tested. Dr. Weisend has also established a relationship with the newly recruited epileptologist at the University of New Mexico, Bruce Fisch, who is excited to begin the scanning of clinical epilepsy patients. Upon the solution of some political and administrative hurdles between the University of New Mexico and MIND the scanning of clinical patients will begin. 


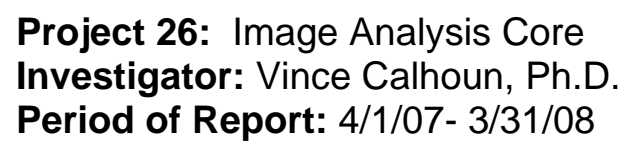

\section{Abstract:}

The image Analysis Core provides algorithm development and infrastructure for automated and large scale processing of the many different data types collected as part of the MIND Research Network. This includes various quality assurance measures in order to evaluate the reliability of the data. We routinely collect multimodal data which includes functional MRI, structural MRI, EEG, MEG, diffusion tensor imaging, spectroscopy, neuropsychological test scores, and diagnostic information. In order to take maximal advantage of the data collected at multiple sites, including the FIRST program and the MCIC program, in addition to the multiple studies conducted locally at the MIND, these data need to be processed in order to optimally utilize the available information. The Core is the vehicle to implement a "pipeline" approach for each modality, using the best processing scheme currently available.

\section{Objectives and Findings:}

1. Technical developments

a. Establishment of automated processing pipeline for MRI, EEG, and MEG scanners, this will involve coordination with the MRI \& MEG/EEG cores to coordinate data transfer of key information from the devices as well as with the IT core to establish the data hierarchy.

-A fully automated pipeline is now functioning for studies performed on the 3T. This includes automatic fMRI analysis, DTI analysis (computing FA maps and tract tracing inputs), VBM analyses (native and spatially normalized segmented images), and Freesurfer analysis. Summary and QA reports are e-mailed to relevant staff for each study.

b. Standardization of quality assurance process and integration of data into the neuroinformatics database

-In conjunction with the neuroinformatics core we have developed QA protocols and data standardization processes. Several R01's were submitted on this topic.

c. Integration of SPM and Freesurfer image analysis capabilities

-We have successfully integrated SPM and Freesurfer.

d. Extension of existing Freesurfer scheme for automated segmentation of brain images including parcellation for cerebral white matter based on proximity to labeled cortical regions, segmentation of sulcal CSF based on combined T1 and T2 image volumes, segmentation of white matter signal abnormalities (WMSA/lesions) based on T1/T2/PD and FLAIR images where available, segmentation and parcellation of the corpus callosum

e. Integrate Path of Interest statistical analysis software (POIstats) into Freesurfer to allow the multimodal investigation of DWI and fMRI data within an accurately defined neuroanatomical framework. 
f. Integrate Freesurfer, SPM, and POIstats into the FIPS analysis stream to allow automated multimodal investigation of DWI and fMRI in the Freesurfer based anatomical framework for large datasets

-We are still working on aims d-f with MGH but have not completed it (delays occurred in subcontract negotiations).

g. Support 3.0T Siemens implementation of efficient MRI acquisitions for $1.0 \mathrm{~mm}$ isotropic scans with comparable distortions for critical contrasts including T1, T2, T2*, and PD to be used for quantitative morphometry

-We have successfully implemented custom sequences from MGH on our 3T scanner and routinely collect $1.0 \mathrm{~mm}$ isotropic $\mathrm{T} 1 \& \mathrm{~T} 2$ scans.

h. Completion of the MCIC data processing for the initial validation and publication of these advanced developments where appropriate

-We have made very good progress on the MCIC data analysis. Almost 20 manuscripts have been prepared and/or submitted this year. In addition several R01's were submitted to do additional analyses, one is already funded.

2. Training

a. Advanced training and technical support for the implemented pipelines and software tools for the MIND personnel and collaborators

-We have established a wiki page and have provided several training sessions with MIND PI's already to disseminate information on the automated pipelines.

b. Support for the MIND investigators and collaborators who are deploying these methods for use on MIND funded studies

-We have performed automated analysis and support for almost all of the studies implemented on the $3 \mathrm{~T}$ scanner. 


\title{
Project 27: Collaboratory Neuroinformatics Core \\ Investigator: $\mathrm{H}$. Jeremy Bockholt \\ Period of Report: 4/1/07-3/31/08
}

\begin{abstract}
:
Through past DOE sponsorship, the MIND Institute has developed a comprehensive, web-based solution for managing the mental health research data sources collected on several thousand research subjects who have participated in clinical neuroimaging projects over the past 5 years. This infrastructure effort will expand, enhance, and refine these tools, promote interoperability, and provide full support of genetic, MEG, EEG, physiology, and behavioral data sources. Finally, statistical tools will be integrated into our turnkey solution to provide a comprehensive quality assurance capability. The chief goal of this effort is to provide an infrastructure for data acquisition, archiving, querying, retrieval, integration and management of the clinical neuroscience research activities of the MIND Research Network.
\end{abstract}

\section{Objectives and Findings:}

We have made great progress the past 12 months. The MIND neuroinformatics core currently manages and maintains a research database that has 186 active users from 5 sites, 5740 human participants have been consented and enrolled into one or more of 149 independent research studies during which 6894 neuroimaging scans have been performed and 22040 clinical assessments have been acquired from 348 forms of assessments.

We have worked with our MIND investigators, partners, and collaborators this past year to greatly enhance the capabilities of the neuroinformatics core. We continue to support the collaborative needs of the MCIC program, and as MIND launches the FIRST program, we will work with FIRST investigators to support the tools needed for collaboration and exchange of data for that project.

We have improved data acquisition efforts through the use of DCMTK product. This permits real-time transfer of DICOM scan data to the research data hierarchy and MIND database. A real-time scan annotation tools was developed for researchers to annotation their structural and functional imaging data as it is acquired.

We have built better subject data management tools that permit secure access to subject metadata, and provide flexible enrollment capabilities. We have implemented a neuroradiological review application that permits a radiologist to review neuroimaging structural scans and provide investigators with automated reports of results of reviews in the form of a PDF document that can be also sent to human research participant or their health care provider when non-incidental findings are encountered.

We have significantly enhanced our data mining capability by building a web-based data querying tool. This tools permits investigators and researchers to query all of the data sources that are appropriate to share within their own studies, but also across all MIND studies where sharing is allowed.

We have improved quality assurance efforts by providing tools for real-time scan annotation (mentioned above), but also providing a data dictionary for clinical assessments for logical checking data following double-entry and screening for conflicts. We have also built a system for providing control charts on multivariate data (such as results from structural imaging analyses). We have a planned manuscript (Williams first author) on this topic and plan to expand this approach into an enterprise quality assurance program for MIND during future efforts.

The scope of this project was essentially to establish a core resource for MIND investigators to have access to world class neuroinformatics tools. We will continue to build, refine, and provide these tools 
and plan to disseminate our findings in several planned manuscripts, as well as, secure external funding of ongoing efforts with the plan to share our open source tools and approach with the scientific community.

\section{Manuscripts in Progress:}

Bockholt, H.J., Scully, M., Scott, A., Lane, S., Ling, J., Williams, S. Calhoun, V. A software process optimized for neuroinformatics. (In preparation for submission April 30, 2008 to Neuroinformatics).

Bockholt, H.J., Gollub, R., Lauriello, J, Lim, K., Clark, V., Calhoun, V., Schulz, S.C., Andreasen, N.C. The MIND Clinical Imaging Consortium as a case study for neuroinformatics tools. (In preparation for submission May 15, 2008 to Neuroinformatics).

Williams, S, Huzurbazar, A., Bockholt, H.J., Jung. R. Using Control Charts to Detect Anomalous Morphological Measurements in Brain Imaing. (In preparation for submission March 1, 2008 to Journal of Quality Technology). 


\author{
Project 28: Biomedical Engineering Research Core \\ Investigator: Michael Doty \\ Period of Report: 4/1/07-3/31/08
}

\begin{abstract}
:
The purpose of the Biomedical Engineering Research Core is to design, develop, and create engineering projects and devices that help facilitate DOE and other research projects for MIND Central researchers. These engineering projects and devices include the MIND Input Device which improves the way in which subject responses are acquired during functional MRI (fMRI) and MEG experiments. The Biomedical Engineering Research Core works closely with all other cores, as well as, individual investigators, administrators, and research staff within MIND Central to provide technical engineering support. In cooperation with the other cores, the current major focus is to provide equipment and support services for uniform stimulus delivery equipment for MIND Central. This grant provides funding for much of the Biomedical Engineering Research Core Personnel and projects at the MIND Institute.
\end{abstract}

\title{
Objectives and Findings:
}

1. Install, maintain, train, and troubleshoot stimulus delivery equipment for Siemens Sonata 1.5 Tesla MR scanner, Siemens Trio 3.0 Tesla MR scanner, Siemens Avanto Mobile 1.5 Tesla MR scanner, VSM Medtech 275-Channel MEG scanner, Cortech ActiveTwo 128+8 Channel EEG/ERP System, and MIND mock MR scanner including stimulus computers, visual display systems, audio systems, subject response devices, and patient monitoring systems. In conjunction with this objective, the Core supported and ensured the integrity of data resulting following streams of effort:

- Siemens Sonata 1.5 Tesla MR system: 332 scans

- Siemens Trio 3.0 Tesla MR system: 298 scans

- Siemens Avanto Mobile 1.5 Tesla MR system: 700 scans

- $\quad$ VSM Medtech 275-Channel MEG: 154 scans

2. The stimulus delivery systems were successfully upgraded for the Siemens Trio 3.0 Tesla MR scanner and Siemens Avanto Mobile 1.5 Tesla MR scanner. The MEG scanner will be done in 2008. These upgrades enable MIND researchers to collect functional imaging data across multiple modalities. All three systems received the exact same high performance stimulus delivery PC computers to minimize timing and incompatibility issues, along with Avotec MRI-compatible headphone systems and MIND "Input Devices" (10 buttons; Right and Left Hand Models; Sizes Adult Small, Medium, and Large). The Siemens Trio and Avanto scanners each received identical visual projection screens, LCD projectors, custom built eyetracking systems, and Biopac galvanic skin response systems. The mobile stimulus upgrade proved to be most difficult due to the space limitations inside the coach/trailer of the mobile MRI scanner. By working with the coach manufacturer and advance planning, the Biomedical Engineering Core team was able to manage an effective installation of the required items and ensure the mobile scanner had the same stimulus delivery features and functionality as its counterparts at MIND Central.

3. Michael Doty continued to develop and maintain the MIND Input Device, an fMRI-compatible cast that is used to record subject responses to stimuli while undergoing a scan. Currently, there are 12 sets of devices in use by the MIND and partner sites. In the past year, MIND broadened patent protection for the MIND Input Device (US Patent Number 7,039,266) to cover several recent improvements, including a button-less version that uses the subject's finger as the switching mechanism. In order to overcome obstacles associated with the strong magnetic environment of the MRI scanners, all ferrous and metal parts were eliminated from the design. 
4. The Biomedical Engineering Core team supported MIND Central investigators on an as needed basis to refine and program stimulus delivery paradigms for their fMRI and MEG experiments. Michael Doty has the programming knowledge and expertise to program paradigms that range from simple to complex using commercially available Stimulus Delivery Software (E-Prime and Presentation Software Suites). Every functional paradigm used at the MIND is created and/or tested by the Biomedical Engineering Core. The paradigms are tested to ensure that the display, timing, and data logging parameters are all set up correctly.

New Technologies: As described above

Patents and Inventions: US Patent Number 7,039,266, also see Appendix EEEEEE

\author{
Project 29: Neurogenetics Core \\ Investigator: Kent Hutchison, Ph.D. \\ Period of Report: 4/1/07-3/31/08
}

\begin{abstract}
:
On June 26, 2000, the American researcher Craig Venter and his firm Celera Genomics announced that they had completed a rough draft of the human genome. Since then, the map that comprises the whole of whole of our hereditary information is being steadily filled in. Scientists once hoped that the genes that code for mental illness would be readily identified. Now, given the extravagant one billion base long chain of amino acids that makes up the human blueprint and the often small subtle differences in its layout, we understand the story is much more complex and finding those genes more challenging.
\end{abstract}

Most major mental illnesses are caused by more than one gene. Researchers are organizing hypotheses around the concept of "multiple genes of small effect." Sorting thorough the 30,000 to 80,000 genes on twenty-three chromosomes to find the multiple genes for a specific illness will take time. This effort will move forward through the use of ingenious computer technologies and statistical methods recently developed to sift through gigantic masses of information. In spite of the astonishing magnitude of the task, it is reasonable to expect that within a few years we will probably be able to identify the alleles that predispose a person to development of a mental illness.

Identifying "bad genes" will prove to be not enough. Moving forward, our investigations need to map the complete cascade of processes that shape connections at the genetic and cellular levels and find expression in the chemical and anatomical circuits of the brain. We will need to find out what proteins are produced by various genes, the function of these proteins, and what disruption occurs when their function is abnormal.

In spite of the power of the many tools the MIND Institute has to probe the brain, to move forward in the battle against mental illness we must join our knowledge base in neuroimaging to the knowledge base of the human genome. This synthesis will position our organization to work on the continuum that is the human biological system, in all of its complex dynamics. 


\title{
Objectives and Findings:
}

In 2007, the MIND Institute recruited Kent Hutchison, Ph.D. to bring up a neurogentics capability for the organization. At the center of his laboratory is an Illumina BeadStation for genetic analysis, acquired with DOE funding. This was augmented by an Applied Biosystems 7500 real-time thermocycler. Selected other equipment, standard for DNA extraction, quantification, and storage, rounded out the laboratory equipment acquisitions. These systems are described in more detail under "Equipment," Attachment $\mathrm{C}$ of the proposal.

Project 30: Training Core

Investigator: Vince Clark, Ph.D.

Period of Report: 4/1/07-3/31/08

\begin{abstract}
:
The Training Core provides the administrative and operational mechanism for the Institute to link its research initiatives to the University of New Mexico for the purpose of developing investigators trained in neuroscience, or its elemental disciplines. The Training Core is lead by Dr. Vince Clark, Science Director for The MIND Institute and Associate Professor of Psychology and Neuroscience at the University of New Mexico (UNM). He serves as the central point of contact between the Institute and UNM schools, colleges, departments and faculty, coordinates connections across fields of study, facilitates student placements, oversees the planning of work assignments, and monitors progress of all participants. The MIND Institute has on-going relationships with many UNM faculty, either through employment, joint appointments, or professional services contracts, across a wide array of disciplines. These relationships combine to form a vibrant environment for students to work and develop the basic competencies of professionals in their chosen career path.
\end{abstract}

\section{Objectives and Findings:}

The main objectives of the Training Core are to:

1. Provide opportunities for students to participate in relevant basic and clinical research and gain from mentoring from senior research staff and/or principal investigators;

2. Provide opportunities for students to expand their knowledge through participation in talks, lectures, trainings, seminars, or symposia;

3. Link the Institute to accredited degree programs at the University of New Mexico in order to build a neuroscience capability for the state.

Findings and/or accomplishments for 2007 include:

1. With respect to research opportunities, the MIND supported 14 graduate students, 7 undergraduate students, and 5 post doctoral candidates pursing degrees in Psychology, Electrical and Computer Engineering, and Neuroscience. Student employees are, in addition to their studies, allowed to work up to 20 hours per week during the academic year and full time over the summer. They are compensated in accordance with established pay schedules set by the University consistent with their level of education and experience. In addition, policies provide for graduate students to receive support for health insurance as well as tuition and fees if they work 10 hours per week for greater than one-half of an academic semester. Apart from monetary arrangements, students working at the MIND participate in actual research tasks relevant to the conduct of neuroscientific studies, not clerical or 
administrative duties. These tasks span an array of activities, such as assistance with subject management during scanning to coordination of processing of imaging data. Moreover, their working onsite with more experienced research staff provides the natural openings for discussion and application of concepts, methods, and techniques in a direct real-world experience.

2. Concerning opportunities to participate in talks, the Institute has sponsored and hosted an exciting array of such events, drawing upon local sources, as well as hosting out-of-town speakers. A list of events is provided below. The period February 2007 to February 2008 is provided as representative of activity for one year at the time of writing this report.

3. One Mind-supported graduate student defended his dissertation and successfully earned his degree: 10/23/2007: Cigarette Smoking and FMRI of Schizophrenia, presented by Leonard Leyba, Neuroscience Department

\section{MIND-Sponsored Talks/Lectures/Trainings/Symposia}

02/08/2007: Virtual Lesions: Elucidating Spatial Attention and Motor Intention in Posterior Parietal Cortex, Tamara Knutsen, Caltech.

02/10/2007: Art \& Artifice of Science Panel Discussion in conjunction with an exhibition at the Museum of Fine Arts in Santa Fe and 516 ARTS in Albuquerque. The exhibits explored the intersection of art and science by examining the work of contemporary artists from New Mexico and beyond, who use the language, look and technologies of science in their work.

02/14/2007: Alcohol and Tobacco Dependence: Leveraging the Human Genome to Develop More Effective Treatments, Kent Hutchison, Department of Psychology, University of Colorado.

02/18-21/2007:_Grand Challenges in Neural Computation: Measurement, Analysis, \& Modeling of Cellular and Network Dynamics, Santa Fe, NM, organized by Los Alamos National Laboratory. This workshop addressed the Scientific Grand Challenges required for quantitatively understanding the nature of computation in the brain and its application toward more powerful neuromimetic computing.

02/22/2007: Molecular and Cellular Landscape of the Brain: The Allen Brain Atlas and Beyond, Ed Lein, Director of Neuroscience, Allen Institute for Brain Science, Seattle, Washington.

\section{5/08/2007: May Symposium on Human Brain Development}

Technology development

- $\quad$ Real-time MRI movement correction method (Andre van der Kouwe, MGH)

- High-resolution MRS in developing brain (Ivan Tkac, CMRR/Minnesota)

- Diffusion Optical Tomography (DOT) (David Boas, MGH)

- MEG-babySQUID integration (Matti Hämäläinen, MGH)

- BabySQUID data analysis with Brainstorm (John Mosher, LANL)

- Genetic approaches (Bill Beavis, NCGR)

Brain injury: Human studies

- Brain injury in newborns and infants (Ellen Grant)

- Brain injury studies with DOT (Maria Franceschini, MGH)

- BabySQUID study of epilepsy (Julia Stephen, UNM)

- Brain injury: Animal studies

- Brain network in juvenile swine after focal injury (M. Tanosaki, BRaIN/UNM)

- Mechanisms of epilepsy (Wolfgang Mueller, BRaIN/UNM)

Autism: Human studies

- Characterization of autism (Martha Herbert, MGH)

- Characterization of autism (Tal Kenet, MGH) 
- Sensory integration (Julia Stephen, UNM)

- Autism: Animal studies

- $\quad$ Mouse model of autism (Xinyu Zhao, UNM)

04/06/2007: A Data-Driven Approach to Analyzing Functional MRI and Single Nucleotide Polymorphism (Mini-series of Bioengineering \#4), Jingyu Liu, Department of Electrical and Computer Engineering, University of New Mexico.

05/08/2007: National Children's Mental Health Awareness Day Reception, Michelle Lujan Grisham, New Mexico Secretary of Health.

05/10/2007: Giftedness, Neural Plasticity, and the Relationship between Reasoning and Attention in the Human Brain, Layne Kalbfleisch, Krasnow Institute/CEHD, George Mason University, Fairfax, Virginia.

05/12/2007 Living with Fibromyalgia documentary and panel discussion, sponsored by the Mind and the Pain Research Group.

06/19/2007: Brain on a Chip: An Engineer's Challenge, Bruce C. Wheeler, Bioengineering Department, University of Illinois.

07/12/07: Neuropsychological Assessment and Rehabilitation: The Efficacy/Effectiveness of EEG Biofeedback (Neurofeedback) for the Treatment of Seizure Disorders, ADHD, Anxiety Disorders, Mood Disorders, Substance Dependence, Traumatic Brain Injury (TBI), and Autism Spectrum Disorder, Harold L. Burke, EEG Spectrum International Inc. and The Brain Therapy Center.

08/17/2007: Brain Correlates of Cognitive and Personality Functioning in Human Volunteers, Rex Jung, Research Scientist, The MIND Institute.

08/22/2007: Investigation of the Neural Mechanisms Underlying facial Emotion Recognition, Robert Thoma, Department of Psychiatry, University of New Mexico.

08/28/2007: MRS Technical Development, Chuck Gasparovic, Department of Neurology, University of New Mexico.

09/07/2007: High Speed MR Spectroscopic Imaging, Stefan Posse, Department of Psychiatry, University of New Mexico.

09/20/2007: Fusion of Multi-Task And Multi-Modal Brain Imaging Data: An Integrated Approach and Several Examples, Vince Calhoun, Director of Image Analysis of MR Research and Electrical \& Computer Engineering, University of New Mexico.

10/02/2007: Distinguishing dementia subtypes with FMRI and DTI, Joseph Sadek, Neuropsychologist, New Mexico VA Health Care System and Assistant Professor, Department of Psychiatry University of New Mexico.

10/10/2007: Frontostriatal Interactions in Reinforcement Learning, Decision Making, and Addiction, Eric Claus, Postdoctoral Fellow, The MIND Institute.

10/17/2007: Cigarette Smoking and FMRI, Leonard Leyba, Research Scientist, The MIND Institute. 


\title{
Project 31: Information Technology Core \\ Investigator: Jeremy Lawrence \\ Period of Report: 4/1/07-3/31/08
}

\begin{abstract}
:
The Information Technology Group supports MRN Investigators and their partners with a secure and reliable computing environment for their research as well as all administrative functions (Payroll, Accounting, HR and Grant Administration). In the past year, the computing environment grew from approximately 200 to 300 scientific and administrative workstations. In addition, the following are maintained: application and compute servers, core services (DNS, Mail, Web, etc.), and a locally managed gigabit Ethernet network based in a centralized high availability data center. The data center itself is secured physically by signature controlled keys and proximity card readers while uninterruptible power supplies and generators guarantee emergency power and cooling. The heart of the data center is a 70 Terabyte research data storage and backup system. This system consolidates all research data to facilitate easy access and retrieval and simultaneously supports transparent and uniform file access across Linux, Macintosh and Windows systems. The storage system uses a fiber channel network to transfer data up to 2.5 gigabytes per second and provides instant data recovery using snapshots. The data center also houses an Overland Neo 8000 tape library for backup and long term storage. Off site backups are also performed for disaster recovery and archival. This enterprise class storage management is key to the success of the organization considering the current growth rate of 2.2 Terabytes of data per month.
\end{abstract}

The IT Core works closely with the other local Cores for capacity planning, performance tuning and general computing support. The computing environment is also available to MRN Partners with active collaboration agreements. All computer and network access is governed by a uniform Acceptable Use Policy signed by all users and reiterated by a banner at login.

The IT Core assists Investigators in hardware specification and software license compliance where applicable and all IT hardware and software purchases must be approved by the IT Director. Dollar amounts and signing authority for purchase approval are the same as those for the organization as a whole. The Core's budget is established cooperatively between the Investigators, CFO and IT Group itself.

\section{Objectives and Findings:}

The IT group's efforts over the past 12 months have focused on uniform Linux deployment, high performance computing, network redundancy, server consolidation and data storage expansion.

\section{Linux Deployment}

This year, the IT Group completed a uniform deployment of Linux across all research workstations. The standard environment has stabilized the application environment allowing researchers to collaborate more effectively. All tools are available on all workstations easing cooperation between groups.

The Linux servers of the Neuroinformatics core are also undergoing a gradual conversion to a uniform release and 64-bit desktop images will be deployed in Q1 2008.

\section{High Performance Computing}

In December of 2007 a new 36 processor Linux compute cluster computer was brought online and began processing fMRI scans using the Freesurfer algorithms. FreeSurfer is a set of automated tools for reconstruction of the brain's cortical surface from structural MRI data, and overlay of functional MRI data onto the reconstructed surface. 
Within 5 days the compute cluster had completed what would have previously taken 3 employees a month to do, more than paying for the cost of the system immediately. The system has since been updated to run several bioinformatics codes in order to process data from the MRN Genetics Lab (Hutchison) as it becomes available.

The Neuroinformatics Group (Bockholt) has teamed with the Magnetic Resonance Imaging Core (Calhoun) to enable automated analysis of MRI data as it is collected using this cluster. The compute power can be expanded easily with $1 \mathrm{U}$ rack mountable commodity hardware as needs increase.

Network Redundancy and Upgrades

Continued improvements in network infrastructure continued in 2007 with the installation of new redundant fiber links between research workstations and the data center.

\section{Server Consolidation}

In an effort to provide higher availability of core services (DNS, DHCP, Directory and Authentication Services etc.) and offer enhanced disaster recovery the IT Group has implemented a server virtualization scheme based on the VMWare ESX virtualization software. MRN currently has about 25 separate pieces of hardware dedicated to a variety of applications or services. By consolidating these into 3 pieces of fully redundant hardware running VMWare we can save power and space while reducing the amount of cooling required in the data center. Legacy servers are gradually being converted to Virtual Machines that run on this hardware and offer the following benefits: each virtual machine represents a complete system, with processors, memory, networking, storage and BIOS; multiple virtual machines can share physical resources and run side by side on the same server; operating systems and applications can run unmodified in virtual machines; and the virtualized systems can be backed up more easily and migrated or restored quickly in the event of minor or catastrophic failures.

\section{Storage Expansion}

Our research data has grown at a slower rate than anticipated last year, but quickly enough to justify a new strategy for managing that growth. On average we are seeing a growth rate of approximately $2.2 \mathrm{~TB} /$ month. However, new computing resources such as the Linux cluster detailed above are enabling researchers to write and win grants that create larger and larger data sets. In order to accommodate growth and reduce costs, we implemented the 128-bit Zettabyte File System (ZFS) from Sun Microsystems on inexpensive Serial ATA drives. The current system is 32TB in size and is expandable to a 128TB store. With minor modifications we can grow the storage system to beyond 192TB in a single "pool". This move to ZFS will allow us to implement future growth and backup strategies much more quickly and inexpensively than we had in the past with proprietary filer hardware and software. 


\section{Publications}

\section{New Mexico 2007}

Adali T \& Calhoun VD. (2007). Complex ICA of Brain Imaging Data. IEEE Signal Proc. Magazine. 24: 136-139.

Assaf M, Johnson M, Schultz R, Sahl R, Calhoun VD, Hendler T \& Pearlson GD. Abnormal Brain Activation During Implicit Mentalization in Autism Spectrum. Society of Biological Psychiatry, 2007.

Astur RS, Germain S, Baker E, Calhoun VD, Pearlson GD \& Constable RT. (In Press). ICA of fMRI Radial Arm Maze. CNS Spectrum.

Benes, F. and Paré-Blagoev, E. J. (2007). Brain Bases of Dyslexia. Mind, Brain and Education in Reading Disorders. In K. W. Fischer, J. H. Bernstein, \& M. H. Immordino-Yang (Eds.),Cambridge, U.K.: Cambridge University Press.

Blagoev KB, Mihaila B, Travis BJ, Alexandrov LB, Bishop AR, Ranken D, Posse S, Gasparovic C, Mayer AR, Aine CJ, Ulbert I \& Halgren E. (2007). Modeling the Magnetic Signature of Neuronal Tissue. Neurolmage. 37: 137-148.

Burge J, Lane T, Link H, Qiu S, Clark VP. (In Press). Discrete Dynamic Bayesian Network Analysis of fMRI Data. Human Brain Mapping.

Bustillo JR, Rowland LM, Jung RE, Brooks WM, Qualls C, Hammond R, Hart B, \& Lauriello J. (In Press). Proton Magnetic Resonance Spectroscopy During the First Year of Antipsychotic Treatment in Schizophrenia. Neuropsychopharmacology. 2007 Dec 19.

Calhoun VD \& Pearlson GD. (In Press). Recent Developments in Brain Imaging of Schizophrenia: A Selective Review. Neuroscience Imaging.

Calhoun VD, Pearlson GD, Maciejewski P \& Kiehl KA. (In Press). Temporal Lobe and 'Default' Hemodynamic Brain Modes Discriminate Between Schizophrenia and Bipolar Disorder. $\underline{\text { Hum. Brain }}$ Map.

Calhoun VD, Pearlson GD, Hillary F \& DeLuca J. (2007). Alcohol Intoxication Effects on fMRI Activation. Functional Neuroimaging in Clinical Populations. Guilford Press, 2007.

Calhoun VD \& Adali T. ICA for Fusion of Brain Imaging Data. Signal Processing Techniques for Knowledge Extraction and Information Fusion, D. Mandic, Ed.: Springer, 2007.

Colom R, Jung RE \& Haier RJ. (In Press). General Intelligence and Memory Span: Evidence for a Common Neuro-anatomic Framework. Cognitive Neuropsychology.

Correa N, Adali T, \& Calhoun VD. (2007). Performance of Blind Source Separation Algorithms for fMRI Analysis. Mag.Res.Imag. 25:684-694.

Demirci O, Clark VP, \& Calhoun VD. (In Press). A Projection Pursuit Application to Detect Schizophrenia Using fMRI Data. Neurolmage.

Dien J, Franklin MS, Michelson CA, Lemen LC, Adams CL \& Kiehl KA. (In Press). fMRI Characterization of the Language Formulation Area. Brain Research.

Eichele T, Calhoun VD, Moosmann M, Specht K, Jongsma M, Quiroga R, Nordby H \& Hugdahl K. (In Press). Unmixing Concurrent EEG-fMRI with Parallel Independent Component Analysis. Int. J. Psych..

Friedman L, Stern H, Brown GG, Mathalon DH, Turner J, Glover GH, Gollub RL, Lauriello J, Lim KO, Cannon T, Greve DN, Bockholt HJ, Belger A, Mueller B, Doty MJ, He J, Wells W, Smyth P, Pieper S, Kim S, Kubicki M, Vangel M \& Potkin SG. (2007). Test-retest and Between-site Reliability in a Multicenter fMRI Study. Hum Brain Map. 2007.

Garrity A, Pearlson GD, McKiernan K, Lloyd D, Kiehl KA \& Calhoun VD. (2007). Aberrant 'Default Mode' Functional Connectivity in Schizophrenia. Am.J.Psychiatry. 164 (3): 450-457.

Haier RJ \& Jung RE. (In Press). Brain Imaging Studies of Intelligence and Creativity: What Is the Picture for Education? Roeper Review.

Haier RJ, \& Jung RE. (2007). Beautiful Minds (i.e. Brains) and the Neural Basis of Intelligence: Response to Commentaries. Behavioral and Brain Sciences. 30(2): 174-178.

Hamilton DA, Akers KG, Weisend MP, \& Sutherland RJ. (2007). How Do Room and Apparatus Cues Control Navigation in the Morris Water Task? Evidence for Distinct Contributions to a Movement Vector. J Exp Psychol Anim Behav Process. 33(2):100-14.

Hejnar MP, Kiehl KA, \& Calhoun VD. (2007). Interparticipant Correlations: A Model Free fMRI Analysis Technique. Hum.Brain Map. 28: 860-867. 
Houck JM, Martin T, Bish JP, Moses SN, Woodruff CC, Kičić D \& Tesche CD. (2007). Early Cerebellar Activation Predicts Response Time. International Congress Series. 1300: 413-416.

Johnson W, Jung RE, \& Haier RJ. (In Press). Psychometric Dimensions of Cognition Other Than General Intelligence Correlate to Regional Brain Structure. Intelligence.

Jung RE, \& Haier RJ. (2007). The Parieto-Frontal Integration Theory (P-FIT) of Intelligence: Converging Neuroimaging Evidence. Behavioral and Brain Sciences. 30(2): 135-187.

Li Y, Adali T \& Calhoun VD. (2007). Estimating the Number of Independent Components for fMRI Data. Hum.Brain Map. 28: 1251-1266.

Li Y, Adali T \& Calhoun VD. (In Press). A Feature-Selective Independent Component Analysis Method for Functional MRI. Int. J. Biomed. Imaging.

Lin F-H, Tsai S-Y, Otazo R, Caprihan A, Wald LL, Belliveau JW \& Posse S. (2007). Sensitvity-encoded (SENSE) Proton Echo Planar Spectroscopic Imaging (PEPSI) in Human Brain. Magn. Reson. Med. 57:249-257.

Lin Q, Zheng Y, Yin F, Liang H, \& Calhoun VD. (In Press). A Fast Algorithm for One-Unit ICA-R. Information Sciences.

Liu J, Pearlson GD, Windemuth A, Ruano G, Perrone-Bizzozero NI \& Calhoun VD. (In Press). Combining fMRI and SNP data to investigate connections between brain function and genetics using parallel ICA. Hum.Brain Map.

Liu J, Demirci O \& Calhoun VD. (In Press). A Parallel Independent Component Analysis Approach to Investigate Genomic Influence on Brain Function. IEEE Signal Proc. Letters.

Martin A, Burtner PA, Poole J, \& Phillips JP. (In Press). ICF level changes in a preschooler after constraint-induced movement therapy. American Journal of Occupational Therapy.

Mayer AR, Franco AR, Sanchez N, Ling J \& Canive J. (2007). Head Motion in Neuropsychiatric Imaging Research: The Theory of Excessive Motion in Schizophrenia. Journal of the International Neuropsychological Society. 13: 839-845.

Mayer, A.R., Harrington, D., Adair, J., Stephen, J. \& Lee, R..The neural networks underlying auditory exogenous facilitation and inhibition of return. Cogn Neurosci. 2007 Mar;19(3):455-467.

Meda S, Gelernter J, Gruen JR, Calhoun VD, Meng H, Cope NA \& Pearlson GD. (In Press). Polymorphism of DCDC2 reveals differences in cortical morphology of healthy individuals - A preliminary voxel based morphometry study. Brain Imaging and Behavior.

Moosmann M, Eichele T, Nordby H, Hugdahl K, \& Calhoun VD. (In Press). Joint Independent Component Analysis for Simultaneous EEG-fMRI: Principle and Simulation. Int. J. Psych.

Moses SN, Houck JM, Martin T, Hanlon FM, Ryan JD, Thoma RJ, Weisend MP, Jackson EM, Pekkonen E \& Tesche CD. (2007). Dynamic Neural Activity Recorded From Human Amygdala During Fear Conditioning Using Magnetoencephalography. Brain Res Bull. 71(5):452-60. Epub 2006 Nov 20.

Paré-Blagoev, E.J. The Neural Correlates of Reading Disorders: Functional Magnetic Resonance Imaging. In K. W. Fischer, J. H. Bernstein, \& M. H. Immordino-Yang (Eds.), Mind, Brain and Education in Reading Disorders. Cambridge, U.K.: Cambridge University Press. (2007)

Pearlson GD \& Calhoun VD. (2007). Structural and Functional Magnetic Resonance Imaging In Psychiatric Disorders. Can. J Psychiatry. 52.

Pettegrew JW, Panchalingam K, McClure, RJ, Stanley JA, Perkins K, Muenz LR, He D, Calhoun VD \& Pearlson GD. (In Press). Brain Molecular Effects of Nicotine: Influence of Age and Smoking Status. Journal of Neuroscience.

Philips JP, Sullivan KJ, Burtner PA, Caprihan A, Provost B \& Bernitsky-Beddingfield A. (2007). Ankle Dorsiflexion $\mathrm{fMRI}$ in Children With Cerebral Palsy Undergoing Intensive Body-Weight-Support Treadmill Training: A Pilot Study. Developmental Medicine and Child Neurology. 49:39-44.

Phillips JP. (In Press). Neuroimaging in Cerebral Palsy: A Clearer Vision of Neuroplasticity. Neuropediatrics.

Pihko E, Nevalainen P, Stephen J, Okada Y \& Lauronen L. (2007). Maturation of the Human Somatosensory Cortex From Birth to Adulthood Revealed by Magnetoencephalography. J. Neurosci.

Plis SM, George JS, Jun SC, Pare-Blagoev J, Ranken DM, Wood CC \& Schmidt DM. (2007). Modeling Spatiotemporal Covariance for Magnetoencephalography or Electroencephalography Source Analysis. Physical Review E, 75, 1.

Posse S, Otazo R, Caprihan A, Bustillo J, Chen H, Henry PG, Marjanska M, Gasparovic C, Zuo C, Magnotta V, Mueller B, Mullins PG, Renshaw P, Ugurbil K, Lim KO, Alger JR. (In Press). Proton Echo Planar Spectroscopic Imaging of J-Coupled Resonances in Human Brain at 3 and 4 Tesla. 
Provost B, Dieruf K, Burtner PA, Phillips JP, Bernitsky-Beddingfield A, Sullivan KJ, Bowen CA, \& Toser L. (2007). Endurance and Gait in Children with Cerebral Palsy Following Intensive Body Weight Supported Treadmill Training. Pediatric Physical Therapy. 19:2-10.

Reis D L, Brackett MA, Shamosh NA, Kiehl KA, Salovey P \& Gray JR. (2007). Emotional Intelligence Predicts Individual Differences In Social Exchange Reasoning. Neuroimage. 35: 1385-1391.

Sanfratello L, Caprihan A \& Fukushima E. (2007). Velocity Depth Profile of Granular Matter in Horizontal Rotating Drum. Granular Matter. 9: 1-6.

Skelly LR, Calhoun VD, Meda SA, Kim J, Mathalon DH \& Pearlson GD. (In Press). Diffusion Tensor Imaging in Schizophrenia: Relationship to Symptoms. Schizophrenia Research.

Sorg A, Riedl V, Muhlau M, Calhoun VD, Drzezga A, Forstl H, Kurz A, Zimmer C \& Wohlschlager A. (In Press). Selective Changes of Resting-State Networks in Patients at High Risk for Alzheimer's Disease - an Example for Profiling Functional Brain Disorders. PNAS.

Stephen JM, Romero L, Zhang T, \& Okada Y. (2007). Auditory and Somatosensory Integration in Infants.

Stevens M, Kiehl KA, Pearlson GD \& Calhoun VD. (2007). Functional Neural Circuits for Mental Timekeeping. Hum.Brain Map. 28 (5): 394-408.

Stevens M, Kiehl KA, Pearlson GD \& Calhoun VD. (2007). Functional Neural Networks Underlying Response Inhibition in Adolescents and Adults. Behav Brain Res. 181 (1): $12-22$.

Stevens MC, Pearlson GP \& Kiehl KA (In Press). Hemodynamics of Auditory Oddball Performance in Combined-subtype Attention-Deficit/Hyperactivity Disorder. American Journal of Psychiatry.

Tesche CD, Moses SN, Houck JM, Martin T, Hanlon FM, Jackson E \& Kičić D. (2007). Dynamics of Frontal and Cerebellar Activation During Aversive Conditioning: A MEG Study. International Congress Series. 1300: 437-440

Thoma RJ, Hanlon FM, Petropoulos H, Miller GA, Moses SN, Smith A, Parks L, Lundy SL, Sanchez NM, Jones A, Huang M, Weisend MP \& Cañive JM. (2007). Schizophrenia Diagnosis and Anterior Hippocampal Volume Make Separate Contributions to Sensory Gating. Psychophysiology

Thoma RJ, Hanlon FM, Huang M, Miller GA, Moses SN, Weisend MP, Jones A, Paulson KM, Irwin J \& Canive JM. (2007). Impaired Secondary Somatosensory Gating in Patients With Schizophrenia. Psychiatry Res. 151(3):189-99. Epub 2007.

Wakana S, Caprihan A, Panzenboeck M, Perry M, Fallon J, Gollub RL, Hua K, Zhang J, Jiang H, Dubey P, Blitz A, van Zijl PCM \& Mori S. (2007). Tractography-based Quantitative Analysis of White Matter Tractography: Tracking Protocol, Reproducibility and Tract-specific Quantification of Cerebral Hemisphere. Neuroimage. 36: 830-644.

Weisend MP, Hanlon FM, Montaño R, Ahlfors SP, Leuthold AC, Pantazis D, Mosher JC, Georgopoulos AP, Hämäläinen MS \& Aine CJ. (2007). Paving the Way for Cross-site Pooling of Magnetoencephalography (MEG) Data. International Congress Series. 1300: 615-618.

\section{Massachusetts General Hospital}

Auranen T, Nummenmaa A, Hamalainen MS, Jaaskelainen IP, Lampinen J, Vehtari A, \& Sams M. (2007). Bayesian inverse analysis of neuromagnetic data using cortically constrained multiple dipoles. $\underline{\text { Hum }}$ Brain Map. 8: 979-994.

Ciesielski KT, Hämäläinen MS, Geller DA, Wilhelm S, Goldsmith TE \& Ahlfors SP. (2007). Dissociation between MEG alpha modulation and performance accuracy on visual working memory task in Obsessive Compulsive Disorder. Hum Brain Map. 8:1401-14.

Dickerson B, Feczko E, Augustinack J, Pacheco J, Morris J, Fischl B \& Buckner R. (2007). Differential effects of aging and Alzheimer. Neurobiol Aging. 2007 Sep 13;

Fischl B, Rajendran N, Busa E, Augustinack J, Hinds O, Yeo BTT, Mohlberg H, Amunts K \& Zilles K. (2007). Cortical Folding Patterns and Predicting Cytoarchitecture, Cerebral Cortex. doi:10.1093/cercor/bhm225.

Fischl B \& Wald L. (2007). Phase maps reveal cortical architecture. Proc Natl Acad Sci USA. 104(28):11513-4.

Frye RE, McGraw Fisher J, Witzel T, Ahlfors SP, Swank P, Liederman J \& Halgren E. (In Press). Objective Phonological and Subjective Perceptual Characteristics of Syllables Modulate Spatiotemporal Patterns of Superior Temporal Gyrus Activity. Neurolmage.

Hara K, Lin FH, Camposano S, Foxe DM, Grant PE, Bourgeois BF, Ahlfors SP \& Stufflebeam SM. (2007). Magnetoencephalographic mapping of interictal spike propagation: a technical and clinical report. AJNR Am J Neuroradiol. 28:1486-8. 
Hinds OP, Rajendran N, Polimeni JR, Augustinack JC, Wiggins G, Wald LL, Rosas HD, Potthast A, Schwartz EL \& Fischl B. (2007). Accurate prediction of V1 location from cortical folds in a surface coordinate system. Neurolmage. 2007.10.033.

Jones SR, Pritchett DL, Stufflebeam SM, Hämäläinen M \& Moore CI. (2007). Neural Correlates of Tactile Detection: A Combined Magnetoencephalography and Biophysically Based Computational Modeling Study. J. Neurosci. 27: 10751-64.

Lin FH, Wang FN, Ahlfors SP, Hamalainen MS \& Belliveau JW. (2007). Parallel MRI Reconstruction Using Variance Partitioning Regularization. Magn. Reson. Med. 58:735-44.

Manoach D, Ketwaroo G, Polli F, Thakkar K, Barton J, Goff D, Fischl B, Vangel M \& Tuch D. (2007). Reduced microstructural integrity of the white matter underlying anterior cingulate cortex is associated with increased saccadic latency in schizophrenia. Neuroimage. 37(2):599-610.

Moon SY, Barton JJ, Mikulski S, Polli FE, Cain MS, Vangel M, Hamalainen MS \& Manoach DS. (2007). Where left becomes right: A magnetoencephalographic study of sensorimotor transformation for antisaccades. Neuroimage. 36:1313-23.

Nummenmaa AR, Auranen T, Hämäläinen MS, Jääskeläinen IP, Sams M, Vehtari A \& Lampinen J. (2007). Automatic relevance-determination based hierarchical Bayesian MEG inversion in practice. Neuroimage. 37:876-89.

Nummenmaa A, Auranen T, Hämäläinen M, Jääskeläinen I, Lampinen J, Sams M \& Vehtari A. (2007). Hierarchical Bayesian estimates of distributed MEG sources: theoretical aspects and comparison of variational and MCMC methods. Neuroimage. 2007 Feb 12; [Epub ahead of print].

Postelnicu G, Zollei L, Desikan R, Fischl B. (2007). Geometry driven volumetric registration. Inf Process Med Imaging. 20:675-86.

Sharon D, Hamalainen MS, Tootell RB, Halgren E \& Belliveau JW. (2007). The advantage of combining MEG and EEG: Comparison to fMRI in focally stimulated visual cortex. Neuroimage. 36:1225-35.

Wehner DT, Hämäläinen MS, Mody, M \& Ahlfors SP. (In Press). Head movements of children in MEG: Quantification, effects on source estimation, and compensation. Neurolmage.

Wehner D, Ahlfors SP \& Mody M. (2007). The influence of semantic processing on phonological decisions in children and adults: A magnetoencephalography (MEG) study. J. Speech Lang. Hearing Res. 50:716-31.

Wehner D, Ahlfors SP \& Mody M. (2007). Effects of phonological contrast on auditory word discrimination in children with and without reading disability: A magnetoencephalography (MEG) study. Neuropsychologia. 45:3251-62.

Wisco JJ, Kuperberg G, Manoach D, Quinn BT, Busa E, Fischl B, Heckers S \& Sorensen AG. (2007). Abnormal cortical folding patterns within Broca's area in schizophrenia: Evidence from structural MRI. Schizophrenia Research. (1-3):317-27.

Yu P, Grant PE, Qi Y, Han X, Sgonne F, Pienaar R, Busa E, Pacheco J, Makris N, Buckner RL, Golland P \& Fischl B. (2007). Cortical Surface Shape Analysis Based on Spherical Wavelets. IEEE Transactions on Medical Imaging. 26(4):582-597.

\section{University of Minnesota 2007}

Chamberlain R, Park J-Y, Corum C, Yacoub E, Ugurbil K, Jack C \& Garwood M. RASER: A New Ultrafast Magnetic Resonance Imaging Method. Magnetic Resonance in Medicine. 2007. 58:794799.

Dobre M, Ugurbil K \& Marjanska M. Determination of Blood Longitudinal Relaxation time (T1) at High Magnetic Field Strengths. Magnetic Resonance Imaging. 2007. 25: 733-735.

Du F, Zhu X-H, Qiao H, Zhang X \& Chen W. Efficient In Vivo 31P Magnetization Transfer Approach for Noninvasively Determining Multiple Kinetic Parameters and Metabolic Fluxes of ATP Metabolism in the Human Brain. MRM. 2007. 57:103-114.

Haddadin I, Mclntosh A, Meisamy S, Corum C, Styczynski A, Powell N, Nelson M, Yee D, Garwood M \& Bolan P. Review Article: Metabolite Quantification and High-Field MRS in Breast Cancer. Wiley InterScience 2007.

Henry P-G, Russeth K, Tkac I, Drewes L, Andrews M \& Gruetter R. Brain Energy Metabolism and Neurotransmission at Near-Freezing Temperatures:/In Vivo/ $1 \mathrm{H}$ MRS Study of the Hibernating Mammal. J Neurochem. 2007. 101(6): 1505-1515. 
Im C-H, Gururajan A, Zhang N, Chen N, Chen W \& He B. Spatial Resolution of EEG Cortical Source Imaging Revealed by Localization of Retinotopic Organization in Human Primary Visual Cortex. $\underline{J}$ Neuroscience Methods. 2007. 161:142-154.

Jack C, Marjanska M, Wengenack T, Reyes D, Curran G, Lin J, Preboske G, Poduslo J, Garwood M: Magnetic Resonance Imaging of Alzheimer's Pathology in the Brain of Living Transgenic Mice: A New Tool in Alzheimer's Disease Research. Neuroscientist. 2007. 13: 38.

Mangia S, Tkac I, Logothetis N, Gruetter R, Van de Moortele P-F \& Ugurbil K: Dynamics of Lactate Concentration and Blood Oxygen Level-Dependent Effect in the Human Visual Cortex During Repeated Identical Stimuli. J of Neuroscience Research. 2007. 85: 3340-3346.

Mangia S, Tkac I, Gruetter R, Van de Mortele P-F, Maraviglia B \& Ugurbil K. Sustained Neuronal Activation Raises Oxidative Metabolism to a New Steady-State Level: Evidence from ${ }_{1} \mathrm{H} \mathrm{NMR}$ Spectroscopy in the Human Visual Cortex. J of Cerebral Blood Flow and Metabolism. 2007. 10551063.

Michaeli S, Oz G, Sorce D, Garwood M, Ugurbil K, Majestic S \& Tuite P. Assessment of Brain Iron and Neuronal Integrity in Patients with Parkinson's Disease Using Novel MRI Contrasts. Movement Disorders. 2007. pp 334-340.

Oz G, Seaquist E, Kumar A, Criego A, Benedict L, Rao J-P, Henry P-G, Van De Moortele P-F \& Gruetter R. Human Brain Glycogen Content and Metabolism: Implications on its Role in Brain Energy Metabolism. Am J Physiol. 2007. 292(3):E946-951.

Rao R, Tkac I, Townsend E, Ennis K, Gruetter R \& Georgieff M. Perinatal Iron Deficiency Predisposes the Developing Rat Hippocampus to Greater Injury From Mild to Moderate Hypoxia-Ischemia. $\underline{\mathrm{J}}$ of Cerebral Blood Flow and Metabolism. 2007. 27:729-740.

Shestov A, Valette J, Ugurbil K \& Henry P-G. On the Reliability of ${ }^{13} \mathrm{C}$ Metabolic Modeling with TwoCompartment Neuronal-Glial Models. J Neurosci Res. 2007. 85(15):3294-303.

Source D, Michaeli S, Garwood M: Relaxation During Adiabatic Radiofrequency Pulses. Current Analytical Chemistry. 2007. 3: 239-251.

Tkac I, Dubinsky J, Keene C, Gruetter R \& Low W. Neurochemical Changes in Huntington R6/2 Mouse Striatum Detected by In Vivo ${ }^{1}$ H NMR Spectroscopy. J of Neurochemisty. 2007. 100: 397-1406.

Valette J, Park JY, Grohn O, Ugurbil K, Garwood M \& Henry PG. Spectroscopic Imaging with Volume Selection by Unpaired Adiabatic $\pi$ Pulses: Theory and Application. J Magnetic Resonance. 2007. 189(1):1-12.

Ward K, Tkac I, Jing Y, Felt B, Beard J, Connor J, Schallert T, Georgieff M \& Rao R. Gestational and Lactational Iron Deficiency Alters the Developing Striatal Metabolome and Associated Behaviors in Young Rats. J of Nutrition. 2007. 137:1043-1049.

White T, Kendi ATK, Lehéricy S, Kendi M, Karatekin C, Guimaraes A, Davenport N, Schulz SC \& Lim KO. Diffusion Tensor Imaging in Children and Adolescents with Schizophrenia - A voxel based analysis. Schizophr Res. 2007. 90(1-3):302-307. Epub 2006 Dec 1.

Wilson TW, Leuthold AC, Moran JE, Pardo PJ, Lewis SM \& Georgopoulos AP. Reading in a Deep Orthography: Neuromagnetic Evidence for Dual-mechanisms. Exp Brain Res. 2007.

Zhang N, Liu Z, He B \& Chen W. Noninvasive Study of Neurovascular Coupling During Graded Neuronal Suppression. $\mathrm{J}$ of Cerebral Blood Flow and Metabolism. 2007; 1-11. 


\begin{tabular}{|c|c|c|c|c|c|c|c|}
\hline \multicolumn{8}{|c|}{$\begin{array}{c}\text { Funded Research Proposals } \\
\text { Fiscal } 2007\end{array}$} \\
\hline & $\begin{array}{l}\text { MIND } \\
\text { PI }\end{array}$ & $\begin{array}{l}\text { MIND } \\
\text { CO-PIs }\end{array}$ & $\begin{array}{l}\text { TITLE of } \\
\text { PROJECT }\end{array}$ & $\begin{array}{l}\text { FUNDING } \\
\text { AGENCY }\end{array}$ & $\begin{array}{l}\text { START } \\
\text { DATE }\end{array}$ & DURATION & TOTAL \\
\hline 1 & Jung & & The Neuroscience of Tetris & $\begin{array}{l}\text { Blue Planet } \\
\text { Software Co. }\end{array}$ & $10 / 1 / 2007$ & $1 \mathrm{yr}$. & $\$ 89,540$ \\
\hline 2 & Hutchison & Kiehl & $\begin{array}{l}\text { Subcontract (Bryan): HIV } \\
\text { prevention with adolescents: } \\
\text { Neurocognitive deficits and } \\
\text { treatment response }\end{array}$ & UNM $\quad$ NIH & $9 / 30 / 2007$ & 5 yrs. & $\$ 1,789,646$ \\
\hline 3 & Hutchison & & $\begin{array}{l}\text { Sensitivity to Intravenous } \\
\text { Ethanol: Genetic Determinants }\end{array}$ & $\mathrm{NIH}$ & $9 / 27 / 2007$ & 5 yrs. & $\$ 1,741,886$ \\
\hline 4 & Bockholt & Ling & $\begin{array}{l}\text { Supplement: (Calhoun) } \\
\text { Collaborative Research: } \\
\text { Spatiotemporal Fusion of fMRI, } \\
\text { EEG, and Genetic Data Using } \\
\text { ICA }\end{array}$ & NIBIB & $9 / 25 / 2007$ & $1 \mathrm{yr}$. & $\$ 79,317$ \\
\hline 5 & Mullins & Mayer & Neurochemistry of pain_Istart & $\mathrm{NIH}$ & $9 / 25 / 2007$ & $1 \mathrm{yr}$. & $\$ 247,826$ \\
\hline 6 & Caprihan & $\begin{array}{c}\text { Bockholt } \\
\text { Clark } \\
\text { (Gasparovic) } \\
\text { Mullins } \\
\end{array}$ & $\begin{array}{l}\text { Subcontract (Thoma):Adolescent } \\
\text { Neurodevelopment and Alcohol }\end{array}$ & $\begin{array}{l}\text { UNM } \\
\text { NIAAA }\end{array}$ & 9/25/2007 & 2 yrs. & $\$ 427,766$ \\
\hline 7 & Stephen & & $\begin{array}{l}\text { Subcontract (Aine): Functional } \\
\text { Imaging of Aging and } \\
\text { Alzheimer's Disease }\end{array}$ & $\begin{array}{l}\text { UNM } \\
\text { NIA }\end{array}$ & $7 / 1 / 2007$ & $1 \mathrm{yr}$. & $\$ 32,109$ \\
\hline 8 & Mayer & $\begin{array}{l}\text { Stephen } \\
\text { Clark } \\
\text { Calhoun } \\
\text { Kiehl }\end{array}$ & $\begin{array}{l}\text { Sensory Gating in Cocaine } \\
\text { Abuse }\end{array}$ & $\mathrm{NIH}$ & 8/6/2007 & 1yr. & $\$ 256,974$ \\
\hline 9 & Jung & & Neuroscience of Creativity & $\begin{array}{l}\text { Templeton } \\
\text { Foundation }\end{array}$ & $8 / 15 / 2007$ & 3 yrs. & $\$ 600,770$ \\
\hline 10 & Hutchison & & $\begin{array}{l}\text { Sub (Bryan): Marijuana Use, } \\
\text { Gender, and Adolescent HIV } \\
\text { Sexual Risk }\end{array}$ & UNM $\quad$ NIH & $8 / 1 / 2007$ & $1 \mathrm{yr}$. & $\$ 53,513$ \\
\hline 11 & Hutchison & & $\begin{array}{l}\text { Sub (Bryan): Mediators and } \\
\text { Moderators of Exercise Behavior } \\
\text { Change }\end{array}$ & $\begin{array}{l}\text { UNM } \\
\text { NIH }\end{array}$ & 9/1/2007 & 2 yrs. & $\$ 47,902$ \\
\hline 12 & Hutchison & & 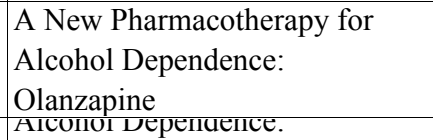 & NIAAA & $8 / 1 / 2007$ & $3.5 \mathrm{yrs}$ & $\$ 2,116,537$ \\
\hline 13 & Hutchison & & $\begin{array}{l}\text { Integrating Genetic and fMRI } \\
\text { Mathodc }\end{array}$ & NIAAA & $8 / 1 / 2007$ & $2.5 \mathrm{yrs}$ & $\$ 1,359,943$ \\
\hline 14 & Filbey & Hutchison & $\begin{array}{l}\text { The Effects of CNR1 on Brain } \\
\text { Function in Cannabis Users }\end{array}$ & NIDA & $8 / 1 / 2007$ & 5 yrs. & $\$ 1,020,807$ \\
\hline 15 & Rasure & Bockholt & $\begin{array}{l}\text { Subcontract (Magnotta): } \\
\text { BRAINS Morphology and } \\
\text { Image Analysis }\end{array}$ & $\begin{array}{l}\text { UIOWA } \\
\text { NINDS }\end{array}$ & $5 / 15 / 2007$ & 3 yrs. & $\$ 253,841$ \\
\hline
\end{tabular}




\begin{tabular}{|c|c|c|c|c|c|c|c|}
\hline 16 & Bockholt & (Gasparovic) & Subcontract (Kikinis): NA-MIC & $\begin{array}{l}\text { NIBIB } \\
\text { Bringham \& } \\
\text { Women's } \\
\text { Hospital }\end{array}$ & $8 / 1 / 2007$ & 2 yrs. & $\$ 380,000$ \\
\hline 17 & Jung & & $\begin{array}{l}\text { Contract for Services- } \\
\text { Neuropsychology Training }\end{array}$ & SNL & $10 / 31 / 2007$ & $1 \mathrm{yr}$. & $\$ 132,000$ \\
\hline 18 & Kiehl & Calhoun & $\begin{array}{l}\text { Abnormal functional } \\
\text { connectivity in psychosis }\end{array}$ & NIH & $7 / 1 / 2007$ & 3 yrs. & $\$ 1,187,181$ \\
\hline 19 & Rasure & Kiehl & $\begin{array}{l}\text { Capital Funds for Mobile } \\
\text { Imaging }\end{array}$ & State of NM & $7 / 1 / 2007$ & $1 \mathrm{yr}$. & $\$ 500,000$ \\
\hline 20 & Hanlon & & Contract with VA & NMVA & $6 / 1 / 2007$ & $1 \mathrm{yr}$. & $\$ 30,874$ \\
\hline 21 & Calhoun & & $\begin{array}{l}\text { Subaward (Pearlson): } \\
\text { Schizophrenia Biomarkers: } \\
\text { Memory, Genes and fMRI } \\
\end{array}$ & NIMH/NIH & $6 / 11 / 2007$ & 5 yrs. & $\$ 128,746$ \\
\hline 22 & Harenski & Kiehl & $\begin{array}{l}\text { Post-Doc Fellowship: } \\
\text { Multimodal Imaging of Social } \\
\text { Emotion and Decision Making in } \\
\text { Psychopathy }\end{array}$ & NRSA & $6 / 12007$ & 2 yrs. & $\$ 49,646$ \\
\hline 23 & Calhoun & Kiehl & $\begin{array}{l}\text { Multivariate methods for } \\
\text { identifying multi-task/ multi- } \\
\text { modal brain imaging biomarkers }\end{array}$ & NIH/NIBIB & $4 / 1 / 2007$ & 4 yrs. & $\$ 1,840,583$ \\
\hline 24 & Kiehl & $\begin{array}{l}\text { Calhoun } \\
\text { Sanjuan } \\
\text { Calhoun }\end{array}$ & $\begin{array}{l}\text { Neurocognitive Changes } \\
\text { Associated with Behavioral } \\
\text { Treatment in Substance Use } \\
\text { Disorders }\end{array}$ & NIDA & $4 / 1 / 2007$ & $3.5 \mathrm{yrs}$. & $\$ 2,276,877$ \\
\hline 25 & Clark & $\begin{array}{c}\text { Jung, Mayer, } \\
\text { Pare-Blagoev, } \\
\text { Hanlon, } \\
\text { Phillips } \\
\text { Bockholt, } \\
\text { Weisend, } \\
\text { Calhoun } \\
\end{array}$ & Accelerated Learning & DARPA & $3 / 1 / 2007$ & $1.5 \mathrm{yrs}$. & $\$ 1,999,692$ \\
\hline 26 & Gasparovic & Phillips & Clinical Scans & UNMH & $2 / 15 / 2007$ & 5 mos. & $\$ 400,000$ \\
\hline 27 & Kiehl & Calhoun & $\begin{array}{l}\text { Neurocognitive assessment of } \\
\text { 'Callous' Conduct Disordered } \\
\text { youth }\end{array}$ & NIMH/NIH & $1 / 1 / 2007$ & 4 yrs. & $\$ 1,380,060$ \\
\hline 28 & Rasure & Et al & $\begin{array}{l}\text { Research \& Development of } \\
\text { Better Diagnostic Tools for } \\
\text { Mental Illness and Neurological } \\
\text { Disorders }\end{array}$ & DOE & $4 / 1 / 2007$ & $1 \mathrm{yr}$. & $\$ 7,000,325$ \\
\hline 29 & Caprihan & Bockholt & $\begin{array}{l}\text { Subaward (Aine): Pilot Project } \\
\text { Awards in Clinical and } \\
\text { Translational Research } \\
\text { "Neuroimaging Successful vs. } \\
\text { Normal Aging" }\end{array}$ & $\begin{array}{l}\text { UNM } \\
\text { CTSD }\end{array}$ & $1 / 1 / 2007$ & 2 yrs. & $\$ 55,630$ \\
\hline
\end{tabular}




\begin{tabular}{|c|c|c|c|c|c|c|c|}
\hline 30 & Hanlon & & $\begin{array}{l}\text { Non-Invasive Assessment of } \\
\text { Lateralized Hippocampal } \\
\text { Function in Patients with } \\
\text { Unilateral Hippocampal } \\
\text { Sclerncic }\end{array}$ & $\begin{array}{c}\text { Epilepsy } \\
\text { Foundation }\end{array}$ & $1 / 1 / 2007$ & 1yr. & $\$ 50,000$ \\
\hline 31 & Kiehl & Calhoun & $\begin{array}{l}\text { ERP and fMRI of Emotion and } \\
\text { Cognition in Psychopathy }\end{array}$ & NIMH/NIH & $2 / 1 / 2007$ & $2 \mathrm{yrs}$ & $\$ 675,268$ \\
\hline
\end{tabular}

\begin{tabular}{|l|r|}
\hline TOTAL AWARDS & $\$ 28,205,259$ \\
\hline
\end{tabular}




\section{MRN Pending Proposals}

\begin{tabular}{|c|c|c|c|c|c|c|c|c|}
\hline $\begin{array}{c}\text { Date } \\
\text { Submitted }\end{array}$ & CO-PIs & TITLE & Customer & $\begin{array}{c}\text { Govt } \\
\text { Client } \\
\end{array}$ & Start Date & Duration & Total Value & IDC To MRN \\
\hline emy & & & & & & & & \\
\hline 18-Jan-08 & Calhoun & $\begin{array}{l}\text { Data Curation and Establishment } \\
\text { of a BIRN-enabled Data Sharing } \\
\text { System for the MIND Clinical } \\
\text { Imaging Consortium (MCIC) - } \\
\text { R01 }\end{array}$ & $\mathrm{NIH}$ & NIH & $12 / 1 / 2008$ & $2 \mathrm{yrs}$ & $\$ 781,568$ & $\$ 281,568$ \\
\hline
\end{tabular}

\section{Calhoun, Vince}

\begin{tabular}{|c|c|c|c|c|c|c|c|}
\hline & $\begin{array}{l}\text { Subcontract: (Stevens) Adolescent } \\
\text { maturation of brain network } \\
\text { integration for executive control } \\
\text { abilities }\end{array}$ & $\begin{array}{l}\text { Hartford } \\
\text { Hospital }\end{array}$ & $\mathrm{NIH}$ & $5 / 30 / 2007$ & $5 \mathrm{yrs}$ & $\$ 70,857$ & $\$ 26,197$ \\
\hline & $\begin{array}{l}\text { Subcontract (Pearlson): } \\
\text { "Hyperscanning"- Using a 2- } \\
\text { person competitive game to } \\
\text { explore theory of mind. }\end{array}$ & Yale & $\mathrm{NIH}$ & $10 / 17 / 2007$ & 4 yrs & $\$ 379,839$ & $\$ 140,434$ \\
\hline \multirow[t]{2}{*}{ Bockholt } & $\begin{array}{l}\text { Subcontract (Thompson): The } \\
\text { Analysis and Classification of } \\
\text { Brain Signals for Covert Speech } \\
\text { Communication }\end{array}$ & $\begin{array}{l}\text { George } \\
\text { Mason U. } \\
\text { CDMRP }\end{array}$ & DOD & $10 / 15 / 2007$ & $5 \mathrm{yrs}$ & $\$ 1,399,980$ & $\$ 517,603$ \\
\hline & $\begin{array}{l}\text { Subcontract: (Pearlson) Alcohol } \\
\text { Use in College Students: } \\
\text { Cognition and fMRI }\end{array}$ & $\begin{array}{l}\text { Hartford } \\
\text { Hospital }\end{array}$ & $\mathrm{NIH}$ & $5 / 31 / 2007$ & $5 \mathrm{yrs}$ & $\$ 46,371$ & $\$ 17,145$ \\
\hline Kiehl & $\begin{array}{l}\text { A unified framework for flexible } \\
\text { brain image analysis }\end{array}$ & NIH/NIBIB & $\mathrm{NIH}$ & $3 / 1 / 2007$ & $5 \mathrm{yrs}$ & $\$ 2,824,845$ & $\$ 842,107$ \\
\hline 26-Nov-07 & $\begin{array}{l}\text { DNA Diagnostics for Minimizing } \\
\text { Neuromuscular Side Effects of } \\
\text { Statins }\end{array}$ & $\begin{array}{l}\text { Genomas, Inc. } \\
\text { (Windemuth) }\end{array}$ & NIH/SBIR & $7 / 1 / 2009$ & $2 \mathrm{yrs}$ & $\$ 100,000$ & $\$ 36,972$ \\
\hline 27-Nov-07 & $\begin{array}{l}\text { Biomarker of Cognitive } \\
\text { Impairment in Chronic Alcoholism }\end{array}$ & $\begin{array}{l}\text { PHRF, Inc. } \\
\text { (Pettegrew) }\end{array}$ & NIH/STTR & $7 / 1 / 2008$ & $2 \mathrm{yr}$ & $\$ 30,000$ & $\$ 11,092$ \\
\hline
\end{tabular}


2007 Progress Report Attachment C

\begin{tabular}{|c|c|c|c|c|c|c|c|c|}
\hline $\begin{array}{c}\text { Date } \\
\text { Submitted }\end{array}$ & CO-PIs & TITLE & Customer & $\begin{array}{c}\text { Govt } \\
\text { Client } \\
\end{array}$ & Start Date & Duration & Total Value & IDC To MRN \\
\hline 27-Nov-07 & & $\begin{array}{l}\text { Molecular Biomarker for } \\
\text { Neurodevelopment }\end{array}$ & $\begin{array}{l}\text { PHRF, Inc. } \\
\text { (Pettegrew) }\end{array}$ & NIH/STTR & $7 / 1 / 2008$ & $2 \mathrm{yr}$ & $\$ 30,000$ & $\$ 11,092$ \\
\hline 05-Feb-08 & $\begin{array}{l}\text { Feng, Capirhan, } \\
\text { Kiehl }\end{array}$ & $\begin{array}{l}\text { Biophysical modeling and } \\
\text { analysis for whole brain complex- } \\
\text { valued BOLD fMRI }\end{array}$ & $\mathrm{NIH}$ & $\mathrm{NIH}$ & $12 / 1 / 2008$ & $5 \mathrm{yrs}$ & $\$ 3,294,053$ & $\$ 981,301$ \\
\hline 18-Feb-08 & & $\begin{array}{l}\text { Subaward: Functional MRI: } \\
\text { Change in Activation or Change } \\
\text { in Structure? (Resubmission) }\end{array}$ & JHU & $\mathrm{NIH}$ & $12 / 1 / 2008$ & $3 \mathrm{yrs}$ & $\$ 220,452$ & $\$ 81,506$ \\
\hline 05-Mar-08 & & $\begin{array}{l}2008 \text { NARSAD Independent } \\
\text { Investigator Award }\end{array}$ & NARSAD & & $9 / 15 / 2008$ & $2 \mathrm{yrs}$ & $\$ 100,000$ & $\$ 8,000$ \\
\hline
\end{tabular}

$\$ 8,496,397 \quad \$ 2,673,449$

\section{Caprihan, Arvind}

\begin{tabular}{|c|c|c|c|c|c|c|c|c|}
\hline & Mayer & $\begin{array}{l}\text { Integrating DTI and fMRI for } \\
\text { Improved White Matter } \\
\text { Parcellation in Cerebral Palsy }\end{array}$ & $\mathrm{NIH}$ & NIH & $7 / 16 / 2007$ & $2 \mathrm{yrs}$ & $\$ 429,548$ & $\$ 112,870$ \\
\hline 05-Feb-08 & Gasparovic & $\begin{array}{l}\text { Developmental Follow-up and } \\
\text { MRI Imaging of Erythropoetin } \\
\text { Treated Infants }\end{array}$ & UNM (Ohls) & $\mathrm{NIH}$ & $1 / 1 / 2009$ & $4 \mathrm{yrs}$ & $\$ 290,647$ & $\$ 107,458$ \\
\hline $18-F e b-08$ & Bockholt & $\begin{array}{l}\text { Subcontract (UNM Phillips) - } \\
\text { WINKS }\end{array}$ & UNM & $\mathrm{NIH}$ & $1 / 1 / 2009$ & $3 \mathrm{yrs}$ & $\$ 288,371$ & $\$ 106,617$ \\
\hline
\end{tabular}

\section{Chen, Hongii}

28-Jan-08

Brain Glutamate and Outcome in

$\mathrm{UNM} / \mathrm{NIH} \quad \mathrm{NIH}$

$7 / 1 / 2008$

5 yrs

$\$ 439,602$

$\$ 162,531$

Schizophrenia

(Bustillo)

$\$ 439,602$

$\$ 162,531$

\section{Clark, Vincent}

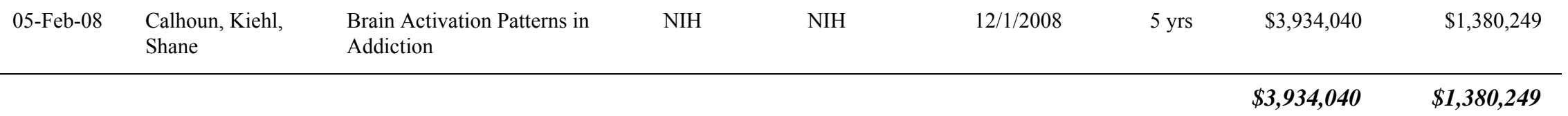


2007 Progress Report Attachment C

\begin{tabular}{ccccccc}
\hline \hline $\begin{array}{c}\text { Date } \\
\text { Submitted }\end{array}$ & CO-PIs & TITLE & Customer & Govt \\
\hline \hline
\end{tabular}

\section{Feldstein Ewing, Sarah}

$\begin{array}{lll}\text { 30-Jan-08 Hutchison } & \begin{array}{l}\text { Neuroimaging of Relapse } \\ \text { Potential in Addiction (R01) }\end{array}\end{array}$

$\mathrm{NIH}$

$12 / 1 / 2008$

5 yrs $\$ 1,980,457$

$\$ 691,378$

$\$ 1,980,457$

$\$ 691,378$

\section{Hutchison, Kent}

\begin{tabular}{|c|c|c|c|c|c|c|c|c|}
\hline & & $\begin{array}{l}\text { Subcontract (Martin): Alcohol } \\
\text { and Substance Use Disorders from } \\
\text { Adolescence through Age } 30\end{array}$ & $\begin{array}{l}\text { U. of } \\
\text { Pittsburgh } \\
\text { NIAAA }\end{array}$ & NIH & $10 / 15 / 2007$ & $5 \mathrm{yrs}$ & $\$ 107,769$ & $\$ 39,845$ \\
\hline & $\begin{array}{l}\text { Clark, Kiehl, } \\
\text { Phillips, Mayer, } \\
\text { Calhoun, Hutchison, } \\
\text { Feldstein }\end{array}$ & $\begin{array}{l}\text { Treatment Development: } \\
\text { Leveraging Imaging and Genetic } \\
\text { Approaches to Addiction (P20 } \\
\text { Center Grant) }\end{array}$ & $\mathrm{NIH}$ & NIH & $10 / 1 / 2008$ & & $\$ 2,400,000$ & $\$ 900,000$ \\
\hline & $\begin{array}{l}\text { Filbey, Calhoun, } \\
\text { Feldstein, Phillips }\end{array}$ & $\begin{array}{l}\text { Effectiveness of Varenicline: } \\
\text { Testing Individual Differences }\end{array}$ & NIDA & NIH & $10 / 4 / 2007$ & $5 \mathrm{yrs}$ & $\$ 3,210,338$ & $\$ 1,186,931$ \\
\hline & & $\begin{array}{l}\text { Subcontract (Patrick): Late } \\
\text { adolescents' health risk behaviors: } \\
\text { Changes, predictors and } \\
\text { consequences }\end{array}$ & $\begin{array}{l}\text { U. of } \\
\text { Pittsburgh }\end{array}$ & NIH & $6 / 20 / 2007$ & $5 \mathrm{yrs}$ & $\$ 122,104$ & $\$ 44,145$ \\
\hline & & $\begin{array}{l}\text { Subcontract (Ito): Multilevel } \\
\text { Analysis of Self-regulation and } \\
\text { Substance Abuse }\end{array}$ & $\begin{array}{l}\text { Univ. of } \\
\text { Colorado NIH }\end{array}$ & NIH & $10 / 26 / 2007$ & $5 \mathrm{yrs}$ & $\$ 216,392$ & $\$ 80,005$ \\
\hline 27-Feb-08 & Feldstein & $\begin{array}{l}\text { Genetic Moderators of fMRI } \\
\text { Response to Naltrexone in Female } \\
\text { Alcoholics }\end{array}$ & UNM & NIH & $1 / 1 / 2009$ & $5 \mathrm{yrs}$ & $\$ 306,993$ & $\$ 113,502$ \\
\hline
\end{tabular}

$\$ 6,363,596$

\section{Jung, Rex}

$\begin{array}{llllll}\text { Subcontract (Kalbfleisch): Pilot } & \begin{array}{l}\text { George } \\ \text { Mason Univ }\end{array} & \text { NSF } & 9 / 21 / 2007 & 1 \text { yr } & \$ 33,379 \\ \begin{array}{l}\text { Project: Objective } \\ \text { Neurophysiological Correlates Of }\end{array} & & & & \$ 12,341 \\ \text { Performance Of Creative IT } & & & & \\ \text { Designers } & & \end{array}$


2007 Progress Report Attachment C

\begin{tabular}{ccccccc}
\hline \hline $\begin{array}{c}\text { Date } \\
\text { Submitted }\end{array}$ & CO-PIs & TITLE & Customer & Client & Start Date Duration Total Value & IDC To MRN \\
\hline
\end{tabular}

$\$ 33,379$

$\$ 12,341$

\section{Kiehl, Kent}

\begin{tabular}{|c|c|c|c|c|c|c|c|c|}
\hline & $\begin{array}{l}\text { Calhoun, Clark, } \\
\text { Hutchison, Shane }\end{array}$ & $\begin{array}{l}\text { Relationship between error } \\
\text { monitoring deficits, dopamine } \\
\text { genes, and risky decision making } \\
\text { in incarcerated drug abusers }\end{array}$ & $\mathrm{NIH}$ & $\mathrm{NIH}$ & $6 / 18 / 2007$ & $2 \mathrm{yrs}$ & $\$ 436,315$ & $\$ 161,315$ \\
\hline 11-Jan-08 & & $\begin{array}{l}\text { Cognitive Neuroscience of Three } \\
\text { Functional Domains of Disgust }\end{array}$ & NSF & NSF & $8 / 1 / 2008$ & $3 \mathrm{yrs}$ & $\$ 850,346$ & $\$ 314,391$ \\
\hline 05-Feb-08 & Calhoun, Clark & $\begin{array}{l}\text { The Cognitive Neuroscience of } \\
\text { Female Psychopathy R01 }\end{array}$ & $\mathrm{NIH}$ & $\mathrm{NIH}$ & $12 / 1 / 2008$ & $5 \mathrm{yrs}$ & $\$ 3,777,692$ & $\$ 1,396,694$ \\
\hline $05-F e b-08$ & & $\begin{array}{l}\text { Kaward - Neuroimaging Youth } \\
\text { with Callous-Unemotional CD \& } \\
\text { Comorbid Substance Abuse }\end{array}$ & U Mass & NIH & $1 / 1 / 2009$ & $3 \mathrm{yrs}$ & $\$ 117,339$ & $\$ 9,388$ \\
\hline
\end{tabular}

\section{$\$ 5,181,692 \quad \$ 1,881,788$}

\section{Lauriello,}

Bockholt, Calhoun,

Caprihan, Clark,

(Gasparovic)

Hanlon, Hutchison,

Jung, Kiehl, Mayer,

Stephen
Neural Mechanisms of
Schizophrenia: Use Of Multiple

Neuroimaging Tools To Examine

Dysfunctions In Neural Integration
NIH

NIH

$10 / 22 / 2007$

5 yrs

$\$ 8,096,629$

$\$ 3,039,712$

$\$ 8,096,629$

$\$ 3,039,712$

\section{Mayer, Andrew}

04-Feb-08

18-Feb-08
Calhoun

Feasibility of Concussion

UNM

3 yrs

$\$ 20,837$

Department

Attentional Dysfunction/Recovery NIH

$\mathrm{NIH}$

$12 / 1 / 2008$

2 yrs

$\$ 451,240$

$\$ 144,097$ 
2007 Progress Report Attachment C

\begin{tabular}{cccccc}
\hline \hline $\begin{array}{c}\text { Date } \\
\text { Submitted }\end{array}$ & CO-PIs & TITLE & Customer & Client & Start Date Duration Total Value \\
IDC To MRN \\
\hline \hline
\end{tabular}

\section{Shane, Matt}

Kiehl Using rt-fMRI to modulate reward NIH
circuits to drug and non-drug
rewards in cocaine abusers

$\mathrm{NIH}$

$10 / 1 / 2008$

5 yrs

$\$ 2,507,257$

$\$ 641,619$

rewards in cocaine abusers

\section{$\underline{\text { Stephen, Julia }}$}

\begin{tabular}{|c|c|c|c|c|c|c|c|}
\hline & $\begin{array}{l}\text { Auditory and Somatosensory } \\
\text { Integration in Toddlers with } \\
\text { Autism }\end{array}$ & CDMRP & DOD & $9 / 25 / 2007$ & $3 \mathrm{yrs}$ & $\$ 373,639$ & $\$ 219,176$ \\
\hline & $\begin{array}{l}\text { Auditory and Somatosensory } \\
\text { Integration }\end{array}$ & $\mathrm{NIH}$ & $\mathrm{NIH}$ & $7 / 16 / 2007$ & $2 \mathrm{yrs}$ & $\$ 446,069$ & $\$ 142,712$ \\
\hline \multirow[t]{2}{*}{ Caprihan, Bockholt } & $\begin{array}{l}\text { Subcontract (Aine) Imaging the } \\
\text { Development of Memory } \\
\text { Strategies in Aging }\end{array}$ & $\mathrm{NIH}$ & $\mathrm{NIH}$ & $4 / 1 / 2008$ & 5 yrs & $\$ 1,036,656$ & $\$ 383,274$ \\
\hline & $\begin{array}{l}\text { Auditory and Somatosensory } \\
\text { Integration in Toddlers with } \\
\text { Typical and Atypical } \\
\text { Development: In Search of an } \\
\text { Early Marker }\end{array}$ & Autism Speaks & NIH? & $7 / 1 / 2008$ & $3 \mathrm{yrs}$ & $\$ 449,559$ & $\$ 40,869$ \\
\hline
\end{tabular}

$\$ 2,305,923$

$\$ 786,031$

Weisend, Mike

\begin{tabular}{|c|c|c|c|c|c|c|c|}
\hline & $\begin{array}{l}\text { Subcontract (Schwindt): Atomic } \\
\text { magnetometer for human } \\
\text { magnetoencephalography }\end{array}$ & SNL & DOE & $10 / 9 / 2007$ & $4 \mathrm{yrs}$ & $\$ 349,052$ & $\$ 129,052$ \\
\hline \multirow[t]{2}{*}{ 26-Feb-08 } & $\begin{array}{l}\text { Cell Assembly Changes in } \\
\text { Learning }\end{array}$ & NSF & NSF & $1 / 1 / 2009$ & $3 \mathrm{yrs}$ & $\$ 1,011,616$ & $\$ 313,452$ \\
\hline & & & & & & $\$ 1,360,668$ & $\$ 442,504$ \\
\hline
\end{tabular}


The MIND Institute

Fixed Asset Summary - 2007

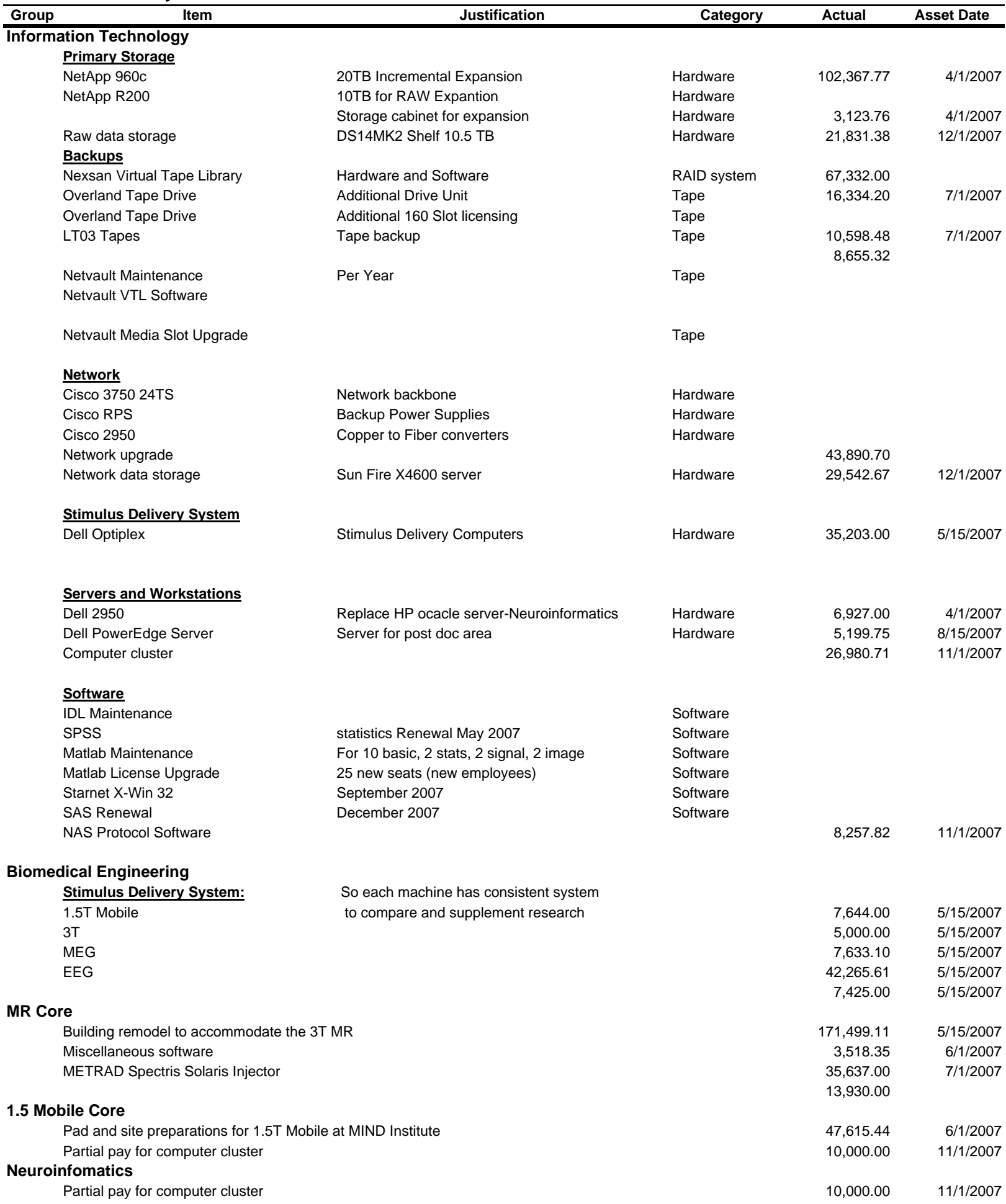




\section{Patents and Inventions:}

US Patent Number 7,039,266

(U.S. Continuation-in-Part Serial No. 11/405,989)

For: Optical Swtiches and Switching Methods

Inventor: Michael Doty, The MIND Institute 